\title{
2017 ESC/EACTS Guidelines for the management of valvular heart disease
}

\section{The Task Force for the Management of Valvular Heart Disease of the European Society of Cardiology (ESC) and the European Association for Cardio-Thoracic Surgery (EACTS)}

\section{Authors/Task Force Members: Helmut Baumgartner* (ESC Chairperson)} (Germany), Volkmar Falk*1 (EACTS Chairperson) (Germany), Jeroen J. Bax (The Netherlands), Michele De Bonis ${ }^{1}$ (Italy), Christian Hamm (Germany), Per Johan Holm (Sweden), Bernard lung (France), Patrizio Lancellotti (Belgium), Emmanuel Lansac' (France), Daniel Rodriguez Muñoz (Spain), Raphael Rosenhek (Austria), Johan Sjögren ${ }^{1}$ (Sweden), Pilar Tornos Mas (Spain), Alec Vahanian (France), Thomas Walther' (Germany), Olaf Wendler ${ }^{1}$ (UK), Stephan Windecker (Switzerland), Jose Luis Zamorano (Spain)

\begin{abstract}
Document Reviewers: Marco Roffi (CPG Review Coordinator) (Switzerland), Ottavio Alfieri' (EACTS Review Coordinator) (Italy), Stefan Agewall (Norway), Anders Ahlsson' (Sweden), Emanuele Barbato (Italy), Héctor Bueno (Spain), Jean-Philippe Collet (France), loan Mircea Coman (Romania), Martin Czerny (Germany), Victoria Delgado (The Netherlands), Donna Fitzsimons (UK), Thierry Folliguet' (France), Oliver Gaemperli (Switzerland), Gilbert Habib (France), Wolfgang Harringer' (Germany), Michael Haude
\end{abstract}

\footnotetext{
* Corresponding authors: Helmut Baumgartner, Division of Adult Congenital and Valvular Heart Disease, Department of Cardiovascular Medicine, University Hospital Muenster, Albert Schweitzer Campus 1, Building A1, 48149 Muenster, Germany. Tel: +492518346110, Fax: +492518346109, E-mail: helmut.baumgartner@ukmuenster.de. Volkmar Falk, Department of Cardiothoracic and Vascular Surgery, German Heart Center, Augustenburger Platz 1, D-133353 Berlin, Germany and Department of Cardiovascular Surgery, Charite Berlin, Charite platz 1, D-10117 Berlin, Germany. Tel: +49304593 2000, Fax: +493045932100, E-mail: falk@dhzb.de.
}

ESC Committee for Practice Guidelines (CPG) and National Cardiac Societies document reviewers listed in the Appendix.

${ }^{1}$ Representing the European Association for Cardio-Thoracic Surgery (EACTS).

ESC entities having participated in the development of this document:

Associations: Acute Cardiovascular Care Association (ACCA), European Association of Cardiovascular Imaging (EACVI), European Association of Percutaneous Cardiovascular Interventions (EAPCI), Heart Failure Association (HFA)

Working Groups: Cardiovascular Pharmacotherapy, Cardiovascular Surgery, Grown-up Congenital Heart Disease, Valvular Heart Disease.

The content of these European Society of Cardiology (ESC) Guidelines has been published for personal and educational use only. No commercial use is authorized. No part of the ESC Guidelines may be translated or reproduced in any form without written permission from the ESC. Permission can be obtained upon submission of a written request to Oxford University Press, the publisher of the European Heart Journal and the party authorized to handle such permissions on behalf of the ESC (journals.permissions@oxfordjournals.org).

Disclaimer. The ESC Guidelines represent the views of the ESC and were produced after careful consideration of the scientific and medical knowledge and the evidence available at the time of their publication. The ESC is not responsible in the event of any contradiction, discrepancy and/or ambiguity between the ESC Guidelines and any other official recommendations or guidelines issued by the relevant public health authorities, in particular in relation to good use of healthcare or therapeutic strategies. Health professionals are encouraged to take the ESC Guidelines fully into account when exercising their clinical judgment, as well as in the determination and the implementation of preventive, diagnostic or therapeutic medical strategies; however, the ESC Guidelines do not override, in any way whatsoever, the individual responsibility of health professionals to make appropriate and accurate decisions in consideration of each patient's health condition and in consultation with that patient and, where appropriate and/or necessary, the patient's caregiver. Nor do the ESC Guidelines exempt health professionals from taking into full and careful consideration the relevant official updated recommendations or guidelines issued by the competent public health authorities in order to manage each patient $s$ case in light of the scientifically accepted data pursuant to their respective ethical and professional obligations. It is also the health professional's responsibility to verify the applicable rules and regulations relating to drugs and medical devices at the time of prescription.

The article has been co-published with permission in the European Heart Journal [10.1093/eurhearti/ehx391] on behalf of the European Society of Cardiology and European Journal of Cardio-Thoracic Surgery [10.1093/ejcts/ezx324] on behalf of the European Association for Cardio-Thoracic Surgery. All rights reserved in respect of European Heart Journal, ( European Society of Cardiology 2017. The articles are identical except for minor stylistic and spelling differences in keeping with each journal's style. Either citation can be used when citing this article.

For permissions, please email journals.permissions@oup.com. 
(Germany), Gerhard Hindricks (Germany), Hugo A. Katus (Germany), Juhani Knuuti (Finland), Philippe Kolh (Belgium), Christophe Leclercq (France), Theresa A. McDonagh (UK), Massimo Francesco Piepoli (Italy), Luc A. Pierard (Belgium), Piotr Ponikowski (Poland), Giuseppe M. C. Rosano (UK/Italy), Frank Ruschitzka (Switzerland), Evgeny Shlyakhto (Russian Federation), lain A. Simpson (UK), Miguel SousaUva' (Portugal), Janina Stepinska (Poland), Giuseppe Tarantini (Italy), Didier Tchétché (France), Victor Aboyans (CPG Supervisor) (France)

The disclosure forms of all experts involved in the development of these guidelines are available on the ESC website http://www.escardio.org/guidelines.

For the Web Addenda which include background information and detailed discussion of the data that have provided the basis for the recommendations see https://academic.oup.com/ eurheartj/article-lookup/doi/10.1093/eurheartj/ehx39 I\#supplementary-data.

\section{ESC CardioMed Textbook Chapter}

Online publish-ahead-of-print 26 August 2017

Keywords Guidelines - Valve disease - Valve surgery - Percutaneous valve intervention • Aortic regurgitation • Aortic stenosis - Mitral regurgitation • Mitral stenosis • Tricuspid regurgitation • Tricuspid stenosis • Prosthetic heart valves

\section{Table of Contents}

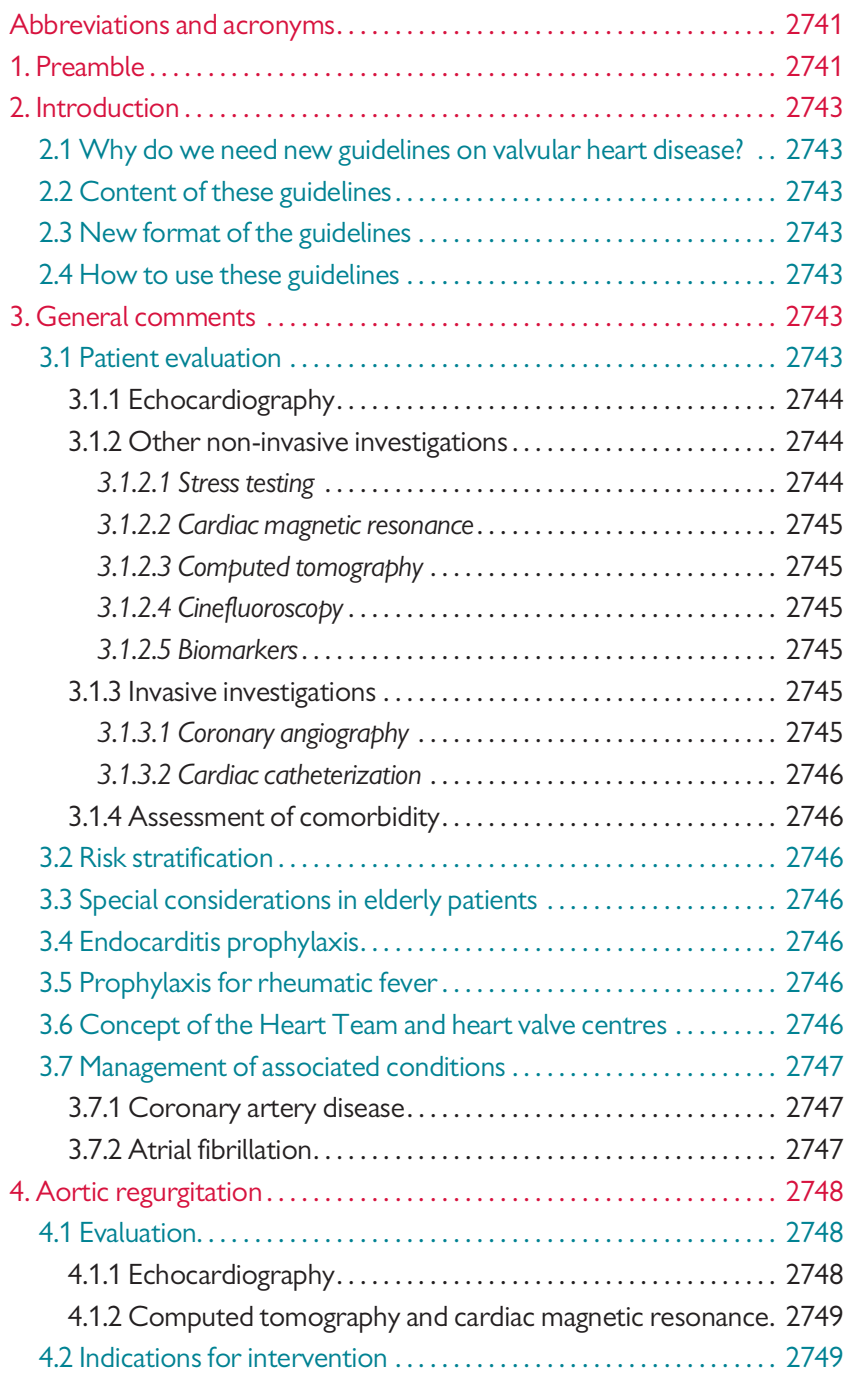

4.3 Medical therapy ............................... 2751

4.4 Serial testing ................................. 2751

4.5 Special patient populations ...................... 2751

5. Aortic stenosis................................... 2751

5.1 Evaluation. ....................................... 2751

5.1 .1 Echocardiography............................ 2751

5.1.2 Additional diagnostic aspects, including assessment of

prognostic parameters ............................. 2753

5.1.3 Diagnostic workup before transcatheter aortic valve implantation . . 2753

5.2 Indications for intervention ............................... 2754

5.2.1 Indications for intervention in symptomatic aortic stenosis. 2756

5.2.2 Choice of intervention mode in symptomatic aortic

stenosis........................................ 2756

5.2.3 Asymptomatic aortic stenosis. .................... 2756

5.3 Medical therapy .................................. 2757

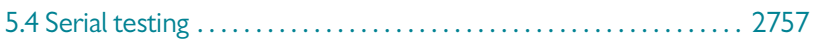

5.5 Special patient populations ......................... 2758

6. Mitral regurgitation .............................. 2758

6.1 Primary mitral regurgitation. ........................ 2758

6.1.1 Evaluation ................................. 2758

6.1 .2 Indications for intervention .................... 2760

6.1 .3 Medical therapy.............................. 2761

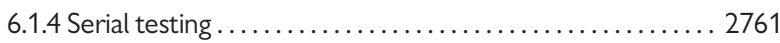

6.2 Secondary mitral regurgitation . ....................... 2761

6.2.1 Evaluation ..................................... 2761

6.2 .2 Indications for intervention ..................... 2761

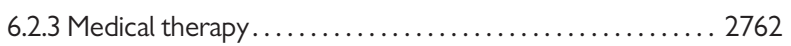

7. Mitral stenosis ..................................... 2762

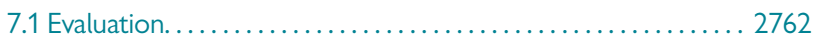

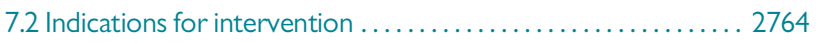

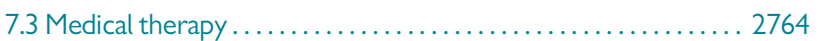

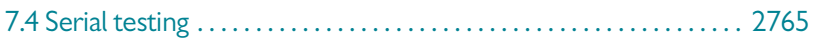

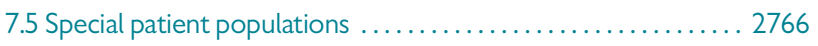

8. Tricuspid regurgitation ........................... 2766

8.1 Evaluation..................................... 2766 
8.2 Indications for intervention ........................ 2766

9. Tricuspid stenosis .............................. 2768

9.1 Evaluation...................................... 2768

9.2 Indications for intervention . ...................... 2768

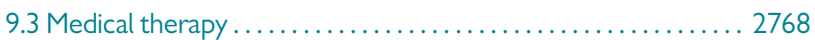

10. Combined and multiple valve diseases .................. 2769

11. Prosthetic valves................................... 2769

11.1 Choice of prosthetic valve. ....................... 2769

11.2 Management after valve intervention.................. 2770

11.2.1 Baseline assessment and modalities of follow-up. ..... 2770

11.2.2 Antithrombotic management. ................... 2771

11.2.2.1 General management ................................ 2771

11.2.2.2 Target international normalized ratio . ............ 2771

11.2.2.3 Management of vitamin K antagonist overdose

and bleeding. .................................. 2771

11.2.2.4 Combination of oral anticoagulants with antiplatelet

drugs ......................................... 2773

11.2.2.5 Interruption of anticoagulant therapy for planned

invasive procedures............................. 2774

11.2.3 Management of valve thrombosis................... 2774

11.2.4 Management of thromboembolism ................. 2777

11.2.5 Management of haemolysis and paravalvular leak ..... 2777

11.2.6 Management of bioprosthetic valve failure ........... 2777

11.2.7 Heart failure. .................................... 2777

12. Management during non-cardiac surgery ................. 2777

12.1 Preoperative evaluation .......................... 2777

12.2 Specific valve lesions .............................. 2778

12.2.1 Aortic stenosis . ................................. 2778

12.2.2 Mitral stenosis . . . . . . . . . . . . . . . . . . . . . . . . . . . . . . 2778

12.2.3 Aortic and mitral regurgitation ..................... 2778

12.3 Perioperative monitoring............................. 2779

13. Management during pregnancy .......................... 2779

13.1 Native valve disease. . . . . . . . . . . . . . . . . . . . . . . . . . . . 2779

13.2 Prosthetic valves ................................... 2779

14. To do and not to do messages from the Guidelines. ......... 2780

15. What is new in the 2017 Valvular Heart Disease Guidelines?. . . 2782

16. Appendix ..................................... 2784

17. References .................................... 2785

\section{Abbreviations and acronyms}

$\begin{array}{ll}\Delta \mathrm{Pm} & \text { Mean transvalvular pressure gradient } \\ \text { 2D } & \text { Two-dimensional } \\ \text { 3D } & \text { Three-dimensional } \\ \text { ABC } & \text { Age, biomarkers, clinical history } \\ \text { ACE } & \text { Angiotensin-converting enzyme } \\ \text { ACS } & \text { Acute coronary syndrome } \\ \text { ARB } & \text { Angiotensin receptor blocker } \\ \text { AVA } & \text { Aortic valve area } \\ \text { BAV } & \text { Balloon aortic valvuloplasty } \\ \text { BNP } & \text { B-type natriuretic peptide } \\ \text { BSA } & \text { Body surface area } \\ \text { CABG } & \text { Coronary artery bypass grafting } \\ \text { CAD } & \text { Coronary artery disease } \\ \text { Cl } & \text { Contra-indication(s) } \\ \text { CMR } & \text { Cardiovascular magnetic resonance }\end{array}$

\begin{tabular}{|c|c|}
\hline CPG & $\begin{array}{l}\text { Committee for Practice Guidelines cardiac } \\
\text { resynchronization therapy }\end{array}$ \\
\hline CT & Computed tomography \\
\hline EACTS & European Association for Cardio-Thoracic Surgery \\
\hline ECG & Electrocardiogram \\
\hline EDV & End-diastolic velocity \\
\hline EROA & Effective regurgitant orifice area \\
\hline ESC & European Society of Cardiology \\
\hline EuroSCORE & $\begin{array}{l}\text { European System for Cardiac Operative } \\
\text { Risk Evaluation }\end{array}$ \\
\hline INR & International normalized ratio \\
\hline IV & Intravenous \\
\hline LA & Left atrium/left atrial \\
\hline LMWH & Low-molecular-weight heparin \\
\hline LV & Left ventricle/left ventricular \\
\hline LVEDD & Left ventricular end-diastolic diameter \\
\hline LVEF & Left ventricular ejection fraction \\
\hline LVESD & Left ventricular end-systolic diameter \\
\hline LVOT & Left ventricular outflow tract \\
\hline MSCT & Multislice computed tomography \\
\hline NOAC & Non-vitamin $\mathrm{K}$ antagonist oral anticoagulant \\
\hline NYHA & New York Heart Association \\
\hline $\mathrm{PCl}$ & Percutaneous coronary intervention \\
\hline PISA & Proximal isovelocity surface area \\
\hline PMC & Percutaneous mitral commissurotomy \\
\hline RV & Right ventricle/right ventricular \\
\hline SAVR & Surgical aortic valve replacement \\
\hline SPAP & Systolic pulmonary arterial pressure \\
\hline STS & Society of Thoracic Surgeons \\
\hline SVi & Stroke volume index \\
\hline TAVI & Transcatheter aortic valve implantation \\
\hline TOE & Transoesophageal echocardiography \\
\hline TTE & Transthoracic echocardiography \\
\hline TVI & Time-velocity interval \\
\hline UFH & Unfractionated heparin \\
\hline VHD & Valvular heart disease \\
\hline VKA & Vitamin $\mathrm{K}$ antagonist \\
\hline$V_{\max }$ & Peak transvalvular velocity \\
\hline
\end{tabular}

\section{Preamble}

Guidelines summarize and evaluate available evidence with the aim of assisting health professionals in selecting the best management strategies for an individual patient with a given condition. Guidelines and their recommendations should facilitate decision making of health professionals in their daily practice. However, the final decisions concerning an individual patient must be made by the responsible health professional(s) in consultation with the patient and caregiver as appropriate.

A great number of guidelines have been issued in recent years by the European Society of Cardiology (ESC) and by the European Association for Cardio-Thoracic Surgery (EACTS) as well as by other societies and organisations. Because of the impact on clinical practice, quality criteria for the development of guidelines have been established in order to make all decisions transparent to the user. The recommendations for formulating and issuing ESC Guidelines can be found on the ESC 
website (https://www.escardio.org/Guidelines/Clinical-Practice-Guide lines/Guidelines-development/Writing-ESC-Guidelines). ESC Guidelines represent the official position of the ESC on a given topic and are regularly updated.

Members of this Task Force were selected by the ESC and EACTS to represent professionals involved with the medical care of patients with this pathology. Selected experts in the field undertook a comprehensive review of the published evidence for management of a given condition according to ESC Committee for Practice Guidelines (CPG) policy and approved by the EACTS. A critical evaluation of diagnostic and therapeutic procedures was performed, including assessment of the risk-benefit ratio. The level of evidence and the strength of the recommendation of particular management options were weighed and graded according to predefined scales, as outlined in Tables 1 and 2.

The experts of the writing and reviewing panels provided declaration of interest forms for all relationships that might be perceived as real or potential sources of conflicts of interest. These forms were compiled into one file and can be found on the ESC website (http:// www.escardio.org/guidelines). Any changes in declarations of interest that arise during the writing period were notified to the ESC and EACTS and updated. The Task Force received its entire financial support from the ESC and EACTS without any involvement from the healthcare industry.

The ESC CPG supervises and coordinates the preparation of new Guidelines. The Committee is also responsible for the endorsement process of these Guidelines. The ESC Guidelines undergo extensive review by the CPG and external experts, and in this case by EACTSappointed experts. After appropriate revisions the Guidelines are approved by all the experts involved in the Task Force. The finalized document is approved by the CPG and EACTS for publication in the European Heart Journal and in the European Journal of
Cardio-Thoracic Surgery. The Guidelines were developed after careful consideration of the scientific and medical knowledge and the evidence available at the time of their dating.

The task of developing ESC/EACTS Guidelines also includes the creation of educational tools and implementation programmes for the recommendations including condensed pocket guideline versions, summary slides, booklets with essential messages, summary cards for non-specialists and an electronic version for digital applications (smartphones, etc.). These versions are abridged and thus, if needed, one should always refer to the full text version, which is freely available via the ESC website and hosted on the EHJ website. The National Societies of the ESC are encouraged to endorse, translate and implement all ESC Guidelines. Implementation programmes are needed because it has been shown that the outcome of disease may be favourably influenced by the thorough application of clinical recommendations.

\section{Table 2 Levels of evidence}

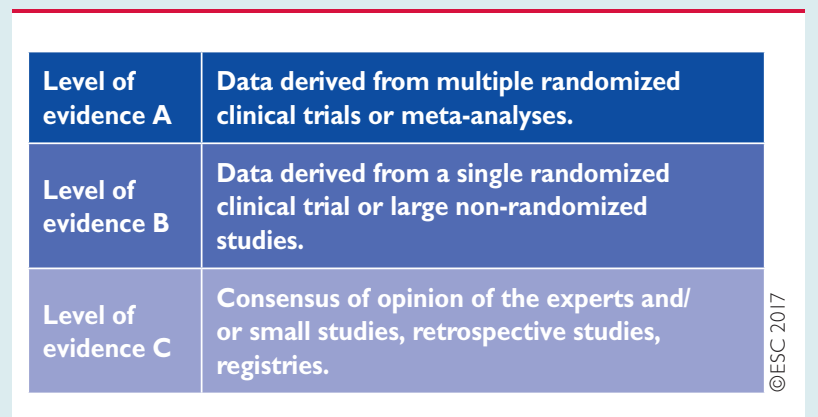

\section{Table I Classes of recommendations}

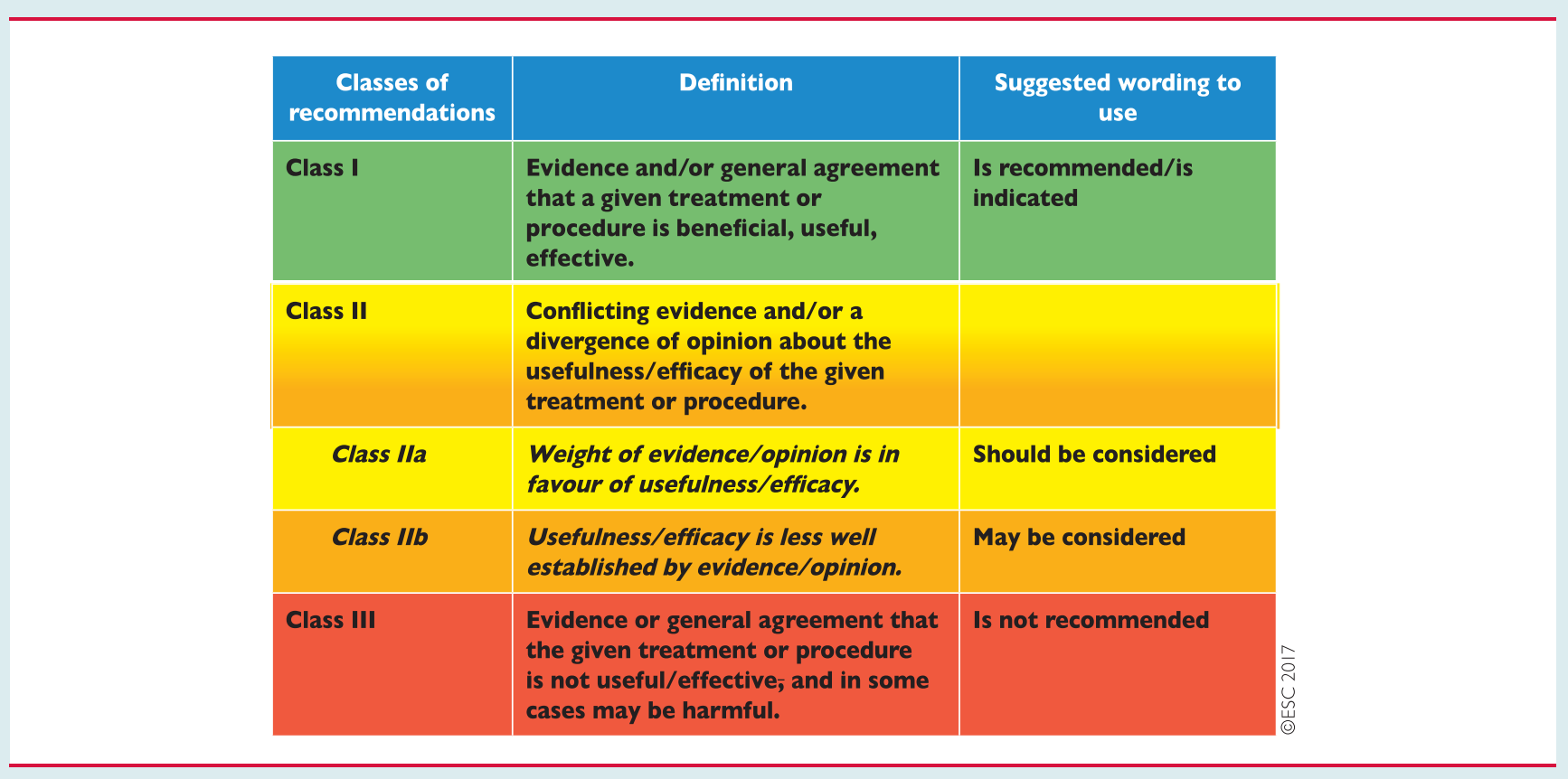


Surveys and registries are needed to verify that real-life daily practice is in keeping with what is recommended in the guidelines, thus completing the loop between clinical research, writing of guidelines, disseminating them and implementing them into clinical practice.

Health professionals are encouraged to take the ESC/EACTS Guidelines fully into account when exercising their clinical judgment, as well as in the determination and the implementation of preventive, diagnostic or therapeutic medical strategies. However, the ESC/ EACTS Guidelines do not override in any way whatsoever the individual responsibility of health professionals to make appropriate and accurate decisions in consideration of each patient's health condition and in consultation with that patient or the patient's caregiver where appropriate and/or necessary. It is also the health professional's responsibility to verify the rules and regulations applicable to drugs and devices at the time of prescription.

\section{Introduction}

\subsection{Why do we need new guidelines on valvular heart disease?}

Since the previous version of the guidelines on the management of VHD was published in 2012, new evidence has accumulated, particularly on percutaneous interventional techniques and on risk stratification with regard to timing of intervention in VHD. This made a revision of the recommendations necessary.

\subsection{Content of these guidelines}

Decision making in VHD involves accurate diagnosis, timing of intervention, risk assessment and, based on these, selection of the most suitable type of intervention. These guidelines focus on acquired VHD, are oriented towards management and do not deal with endocarditis or congenital valve disease, including pulmonary valve disease, as separate guidelines have been published by the ESC on these topics.

\subsection{New format of the guidelines}

The new guidelines have been adapted to facilitate their use in clinical practice and to meet readers' demands by focusing on condensed, clearly represented recommendations. At the end of each section, Key points summarize the essentials. Gaps in evidence are listed to propose topics for future research. The guideline document is harmonized with the simultaneously published chapter on VHD of the ESC Textbook of Cardiology, which is freely available by Internet access (https://academic.oup.com/ eurheartj/article-lookup/doi/10.1093/eurheartj/eh×391\# supplementary-data). The guidelines and the textbook are complementary. Background information and detailed discussion of the data that have provided the basis for the recommendations can be found in the relevant book chapter.

\subsection{How to use these guidelines}

The Committee emphasizes that many factors ultimately determine the most appropriate treatment in individual patients within a given community. These factors include the availability of diagnostic equipment, the expertise of cardiologists and surgeons, especially in the field of valve repair and percutaneous intervention and, notably, the wishes of well-informed patients. Furthermore, owing to the lack of evidencebased data in the field of VHD, most recommendations are largely the result of expert consensus opinion. Therefore, deviations from these guidelines may be appropriate in certain clinical circumstances.

\section{General comments}

The aims of the evaluation of patients with VHD are to diagnose, quantify and assess the mechanism of VHD as well as its consequences. Decision making for intervention should be made by a 'Heart Team' with a particular expertise in VHD, comprising cardiologists, cardiac surgeons, imaging specialists, anaesthetists and, if needed, general practitioners, geriatricians and heart failure, electrophysiology or intensive care specialists. The 'Heart Team' approach is particularly advisable in the management of high-risk patients and is also important for other subsets, such as asymptomatic patients where the evaluation of valve reparability is a key component in decision making. The essential questions in the evaluation of a patient for valvular intervention are summarized in Table 3.

\subsection{Patient evaluation}

Precise evaluation of the patient's history and symptomatic status as well as proper physical examination, in particular auscultation and search for heart failure signs, are crucial for the diagnosis and management of VHD. In addition, assessment of the extracardiac condition-comorbidities and general condition-require particular attention.

Table 3 Essential questions in the evaluation of patients for valvular intervention

\begin{tabular}{|c|}
\hline Questions \\
\hline - How severe is VHD? \\
\hline - What is the aetiology of VHD? \\
\hline - Does the patient have symptoms? \\
\hline - Are symptoms related to valvular disease? \\
\hline $\begin{array}{l}\text { - Are any signs present in asymptomatic patients that indicate a worse } \\
\text { outcome if the intervention is delayed? }\end{array}$ \\
\hline - What are the patient's life expectancy ${ }^{\mathrm{a}}$ and expected quality of life? \\
\hline $\begin{array}{l}\text { - Do the expected benefits of intervention (versus spontaneous } \\
\text { outcome) outweigh its risks? }\end{array}$ \\
\hline $\begin{array}{l}\text { - What is the optimal treatment modality? Surgical valve replacement } \\
\text { (mechanical or biological), surgical valve repair, or catheter } \\
\text { intervention? }\end{array}$ \\
\hline $\begin{array}{l}\text { - Are local resources (local experience and outcome data for a given } \\
\text { intervention) optimal for the planned intervention? }\end{array}$ \\
\hline - What are the patient's wishes? \\
\hline
\end{tabular}




\subsubsection{Echocardiography}

Following adequate clinical evaluation, echocardiography is the key technique used to confirm the diagnosis of VHD as well as to assess its severity and prognosis. It should be performed and interpreted by properly trained personnel. ${ }^{1}$

Echocardiographic criteria for the definition of severe valve stenosis and regurgitation are addressed in specific documents. ${ }^{2-4}$ Recommendations for stenotic lesions are indicated in the corresponding sections and quantification of regurgitant lesions is summarized in Table 4. An integrated approach including various criteria is strongly recommended instead of referring to single measurements. Echocardiography is also key to assess valve morphology and function as well as to evaluate the feasibility and indications of a specific intervention.

Indices of left ventricular (LV) enlargement and function are strong prognostic factors. Pulmonary artery pressure should be estimated as well as right ventricular (RV) function. ${ }^{5}$ Transoesophageal echocardiography (TOE) should be considered when transthoracic echocardiography (TTE) is of suboptimal quality or when thrombosis, prosthetic valve dysfunction or endocarditis is suspected. Intraprocedural TOE is used to guide percutaneous mitral and aortic valve interventions and to monitor the results of all surgical valve operations and percutaneous valve implantation or repair.

\subsubsection{Other non-invasive investigations}

\subsubsection{Stress testing}

The primary purpose of exercise testing is to unmask the objective occurrence of symptoms in patients who claim to be asymptomatic or have non-specific symptoms, and is especially useful for risk stratification in aortic stenosis. ${ }^{8}$ Exercise testing will also determine the level of recommended physical activity, including participation in sports.

Table 4 Echocardiographic criteria for the definition of severe valve regurgitation: an integrative approach (adapted from Lancellotti et al. ${ }^{2,6,7}$ )

\begin{tabular}{|c|c|c|c|c|}
\hline & Aortic regurgitation & \multicolumn{2}{|c|}{ Mitral regurgitation } & Tricuspid regurgitation \\
\hline \multicolumn{5}{|l|}{ Qualitative } \\
\hline Valve morphology & $\begin{array}{c}\text { Abnormal/flail/large coaptation } \\
\text { defect }\end{array}$ & \multicolumn{2}{|c|}{$\begin{array}{c}\text { Flail leaflet/ruptured papillary muscle/ } \\
\text { large coaptation defect }\end{array}$} & $\begin{array}{c}\text { Abnormal/flail/large coaptation } \\
\text { defect }\end{array}$ \\
\hline Colour flow regurgitant jet & $\begin{array}{c}\text { Large in central jets, variable in } \\
\text { eccentric jets }{ }^{\mathrm{a}}\end{array}$ & \multicolumn{2}{|c|}{$\begin{array}{c}\text { Very large central jet or eccentric jet } \\
\text { adhering, swirling, and reaching the } \\
\text { posterior wall of the LA }\end{array}$} & $\begin{array}{c}\text { Very large central jet or eccentric } \\
\text { wall impinging jet }{ }^{\mathrm{a}}\end{array}$ \\
\hline $\mathrm{CW}$ signal of regurgitant jet & Dense & \multicolumn{2}{|c|}{ Dense/triangular } & $\begin{array}{c}\text { Dense/triangular with early peaking } \\
\text { (peak }<2 \mathrm{~m} / \mathrm{s} \text { in massive TR) }\end{array}$ \\
\hline Other & $\begin{array}{c}\text { Holodiastolic flow reversal in } \\
\text { descending aorta (EDV >20 cm/s) }\end{array}$ & \multicolumn{2}{|c|}{ Large flow convergence zone ${ }^{a}$} & - \\
\hline \multicolumn{5}{|l|}{ Semiquantitative } \\
\hline Vena contracta width (mm) & $>6$ & \multicolumn{2}{|c|}{$\geq 7$ (>8 for biplane) ${ }^{b}$} & $\geq 7^{a}$ \\
\hline Upstream vein flow & - & \multicolumn{2}{|c|}{ Systolic pulmonary vein flow reversal } & Systolic hepatic vein flow reversal \\
\hline Inflow & - & \multicolumn{2}{|c|}{ E-wave dominant $\geq 1.5 \mathrm{~m} / \mathrm{s}^{\mathrm{d}}$} & E-wave dominant $\geq 1 \mathrm{~m} / \mathrm{s}^{\mathrm{e}}$ \\
\hline Other & Pressure half-time $<200 \mathrm{~ms}^{\mathrm{f}}$ & \multicolumn{2}{|c|}{ TVI mitral/TVI aortic $>1.4$} & PISA radius $>9 \mathrm{~mm}^{\mathrm{g}}$ \\
\hline Quantitative & & Primary & Secondary ${ }^{h}$ & \\
\hline $\operatorname{EROA}\left(\mathrm{mm}^{2}\right)$ & $\geq 30$ & $\geq 40$ & $\geq 20$ & $\geq 40$ \\
\hline Regurgitant volume (mL/beat) & $\geq 60$ & $\geq 60$ & $\geq 30$ & $\geq 45$ \\
\hline + enlargement of cardiac chambers/vessels & LV & $\mathrm{LV}, \mathrm{LA}$ & & $R V, R A$, inferior vena cava \\
\hline
\end{tabular}

$\mathrm{CW}=$ continuous wave; $\mathrm{EDV}=$ end-diastolic velocity; $\mathrm{EROA}=$ effective regurgitant orifice area; $\mathrm{LA}=$ left atrium/atrial; $\mathrm{LV}=$ left ventricle/ventricular; $\mathrm{PISA}=$ proximal isovelocity surface area; $\mathrm{RA}=$ right atrium/right atrial; $\mathrm{RV}=$ right ventricle; $\mathrm{TR}=$ tricuspid regurgitation; $\mathrm{TVI}=$ time-velocity integral.

${ }^{a}$ At a Nyquist limit of $50-60 \mathrm{~cm} / \mathrm{s}$.

${ }^{b}$ For average between apical four- and two-chamber views.

'Unless other reasons for systolic blunting (atrial fibrillation, elevated atrial pressure)

${ }^{d}$ In the absence of other causes of elevated LA pressure and of mitral stenosis.

e In the absence of other causes of elevated RA pressure.

fPressure half-time is shortened with increasing LV diastolic pressure, vasodilator therapy, and in patients with a dilated compliant aorta, or lengthened in chronic aortic regurgitation.

BBaseline Nyquist limit shift of $28 \mathrm{~cm} / \mathrm{s}$.

${ }^{h}$ Different thresholds are used in secondary mitral regurgitation where an EROA $>20 \mathrm{~mm}^{2}$ and regurgitant volume $>30 \mathrm{~mL}$ identify a subset of patients at increased risk of cardiac events. 
Exercise echocardiography may identify the cardiac origin of dyspnoea. The prognostic impact has been shown mainly for aortic stenosis and mitral regurgitation.

The search for flow reserve (also called 'contractile reserve') using low-dose dobutamine stress echocardiography is useful for assessing aortic stenosis severity and for operative risk stratification in low-gradient aortic stenosis with impaired LV function as well as to assess the potential of reverse remodelling in patients with heart failure and functional mitral regurgitation after a mitral valve procedure. ${ }^{10,11}$

\subsubsection{Cardiac magnetic resonance}

In patients with inadequate echocardiographic quality or discrepant results, cardiac magnetic resonance (CMR) should be used to assess the severity of valvular lesions, particularly regurgitant lesions, and to assess ventricular volumes, systolic function, abnormalities of the ascending aorta and myocardial fibrosis. CMR is the reference method for the evaluation of RV volumes and function and is therefore particularly useful to evaluate the consequences of tricuspid regurgitation. ${ }^{12}$

\subsubsection{Computed tomography}

Multislice computed tomography (MSCT) may contribute to evaluation of the severity of valve disease, particularly in aortic stenosis ${ }^{13,14}$ and of the thoracic aorta. MSCT plays an important role in the workup of patients with VHD considered for transcatheter intervention, in particular transcatheter aortic valve implantation (TAVI), and provides valuable information for pre-procedural planning. Owing to its high negative predictive value, MSCT may be useful to rule out coronary artery disease (CAD) in patients who are at low risk of atherosclerosis.

\subsubsection{Cinefluoroscopy}

Cinefluoroscopy is particularly useful for assessing the kinetics of the occluders of a mechanical prosthesis.

\subsubsection{Biomarkers}

B-type natriuretic peptide (BNP) serum levels are related to New York Heart Association (NYHA) functional class and prognosis, particularly in aortic stenosis and mitral regurgitation. ${ }^{15}$ Natriuretic peptides may be of value for risk stratification and timing of intervention, particularly in asymptomatic patients.

\subsubsection{Invasive investigations}

\subsubsection{Coronary angiography}

Coronary angiography is indicated for the assessment of CAD when surgery or an intervention is planned, to determine if concomitant coronary revascularization is indicated (see following table of recommendations). ${ }^{16}$ Alternatively, coronary computed tomography (CT) can be used to rule out CAD in patients at low risk for the condition.

\section{Management of CAD in patients with VHD (adapted from Windecker et al. ${ }^{16}$ )}

\begin{tabular}{|c|c|c|}
\hline Recommendations & Class $^{a}$ & Level $^{\mathbf{b}}$ \\
\hline \multicolumn{3}{|l|}{ Diagnosis of CAD } \\
\hline 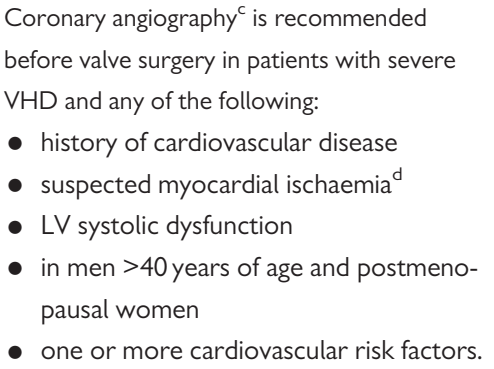 & I & C \\
\hline $\begin{array}{l}\text { Coronary angiography is recommended in the } \\
\text { evaluation of moderate to severe secondary } \\
\text { mitral regurgitation. }\end{array}$ & $\mathbf{I}$ & C \\
\hline $\begin{array}{l}\text { CT angiography should be considered as an } \\
\text { alternative to coronary angiography before } \\
\text { valve surgery in patients with severe VHD and } \\
\text { low probability of CAD or in whom conven- } \\
\text { tional coronary angiography is technically not } \\
\text { feasible or associated with a high risk. }\end{array}$ & Ila & C \\
\hline \multicolumn{3}{|l|}{ Indications for myocardial revascularization } \\
\hline $\begin{array}{l}\text { CABG is recommended in patients with a pri- } \\
\text { mary indication for aortic/mitral valve surgery } \\
\text { and coronary artery diameter stenosis } \geq 70 \% \text {. }\end{array}$ & $\mathbf{I}$ & C \\
\hline $\begin{array}{l}\text { CABG should be considered in patients with a } \\
\text { primary indication for aortic/mitral valve sur- } \\
\text { gery and coronary artery diameter stenosis } \\
\geq 50-70 \% \text {. }\end{array}$ & Ila & C \\
\hline $\begin{array}{l}\mathrm{PCl} \text { should be considered in patients with a } \\
\text { primary indication to undergo } \mathrm{TAVI} \text { and coro- } \\
\text { nary artery diameter stenosis }>70 \% \text { in proxi- } \\
\text { mal segments. }\end{array}$ & Ila & C \\
\hline $\begin{array}{l}\mathrm{PCl} \text { should be considered in patients with a } \\
\text { primary indication to undergo transcatheter } \\
\text { mitral valve interventions and coronary artery } \\
\text { diameter stenosis }>70 \% \text { in proximal segments. }\end{array}$ & Ila & C \\
\hline
\end{tabular}

$C A B G=$ coronary artery bypass grafting; $C A D=$ coronary artery disease; $C T=$ computed tomography; $\mathrm{LV}=$ left ventricular; $\mathrm{MSCT}=$ multislice computed tomography; $\mathrm{PCl}=$ percutaneous coronary intervention; $\mathrm{TAVI}=$ transcatheter aortic valve implantation; $\mathrm{VHD}=$ valvular heart disease

${ }^{\mathrm{a}}$ Class of recommendation.

bLevel of evidence.

CMSCT may be used to exclude CAD in patients who are at low risk of atherosclerosis.

${ }^{\mathrm{d} C}$ Chest pain, abnormal non-invasive testing.

${ }^{e} \geq 50 \%$ can be considered for left main stenosis. 


\subsubsection{Cardiac catheterization}

The measurement of pressures and cardiac output or the assessment of ventricular performance and valvular regurgitation by ventricular angiography or aortography is restricted to situations where noninvasive evaluation is inconclusive or discordant with clinical findings. When elevated pulmonary pressure is the only criterion to support the indication for surgery, confirmation of echo data by invasive measurement is recommended.

\subsubsection{Assessment of comorbidity}

The choice of specific examinations to assess comorbidity is directed by the clinical evaluation.

\subsection{Risk stratification}

Risk stratification applies to any sort of intervention and is required for weighing the risk of intervention against the expected natural history of VHD as a basis for decision making. Most experience relates to surgery and TAVI. The EuroSCORE I (http://www.euroscore.org/ calc.html) overestimates operative mortality and its calibration of risk is poor. Consequently, it should no longer be used to guide decision making. The EuroSCORE II and the Society of Thoracic Surgeons (STS) score (http://riskcalc.sts.org/stswebriskcalc/\#/) more accurately discriminate high- and low-risk surgical patients and show better calibration to predict postoperative outcome after valvular surgery. ${ }^{17,18}$ Scores have major limitations for practical use by insufficiently considering disease severity and not including major risk factors such as frailty, porcelain aorta, chest radiation etc. While EuroSCORE I markedly overestimates 30-day mortality and should therefore be replaced by the better performing EuroSCORE II in this regard, it is nevertheless provided in this document for comparison, as it has been used in many TAVI studies/registries and may still be useful to identify the subgroups of patients for decision between intervention modalities and to predict 1-year mortality. Both scores have shown variable results in predicting the outcomes of intervention in TAVI but are useful for identifying low-risk patients for surgery. New scores have been developed to estimate the risk of 30-day mortality in patients undergoing TAVI, with better accuracy and discrimination, albeit with numerous limitations. ${ }^{19,20}$

Experience with risk stratification is being accumulated for other interventional procedures, such as mitral edge-to-edge repair. It remains essential not to rely on a single risk score figure when assessing patients or to determine unconditionally the indication and type of intervention. Patient's life expectancy, expected quality of life and patient preference should be considered, as well as local resources. The futility of interventions in patients unlikely to benefit from the treatment has to be taken into consideration, particularly for TAVI and mitral edge-to-edge repair. ${ }^{21}$ The role of the Heart Team is essential to take all of these data into account and adopt a final decision on the best treatment strategy. Finally, the patient and family should be thoroughly informed and assisted in their decision on the best treatment option. ${ }^{22}$

\subsection{Special considerations in elderly patients}

Poor mobility, as assessed by the 6-minute walk test, and oxygen dependency are the main factors associated with increased mortality after TAVI and other VHD treatments. ${ }^{23,24}$ The combination of severe lung disease, postoperative pain from sternotomy or thoracotomy and prolonged time under anaesthesia in patients undergoing traditional surgical aortic valve replacement (SAVR) may contribute to pulmonary complications. There is a gradual relationship between the impairment of renal function and increased mortality after valvular surgery, TAVI and transcatheter mitral edgeto-edge repair, ${ }^{25}$ especially when glomerular filtration rate is $<30 \mathrm{~mL} / \mathrm{min}$. Coronary, cerebrovascular and peripheral artery disease have a negative impact on early and late survival after surgery and TAVI. ${ }^{22}$

Besides specific organ comorbidities, there is growing interest in the assessment of frailty, an overall marker of impairment of functional, cognitive and nutritional status. Frailty is associated with increased morbidity and mortality after surgery and TAVI. ${ }^{26}$ The assessment of frailty should not rely on a subjective approach, such as the 'eyeball test', but rather on a combination of different objective estimates. Several tools are available for assessing frailty. $^{23,26,27}$

\subsection{Endocarditis prophylaxis}

Antibiotic prophylaxis should be considered for high-risk procedures in patients with prosthetic valves, including transcatheter valves, or with repairs using prosthetic material and those with previous episodes of infective endocarditis. ${ }^{28}$ Recommendations regarding dental and cutaneous hygiene and strict aseptic measures during any invasive procedures are advised in this population. Antibiotic prophylaxis should be considered in dental procedures involving manipulation of the gingival or periapical region of the teeth or manipulation of the oral mucosa. ${ }^{28}$

\subsection{Prophylaxis for rheumatic fever}

Prevention of rheumatic heart disease should preferably be oriented towards preventing the first attack of acute rheumatic fever. Antibiotic treatment of group A Streptococcus sore throat is key in primary prevention. In patients with rheumatic heart disease, secondary long-term prophylaxis against rheumatic fever is recommended. Lifelong prophylaxis should be considered in high-risk patients according to the severity of VHD and exposure to group $\mathrm{A}$ Streptococcus. $^{29-31}$

\subsection{Concept of the Heart Team and heart valve centres}

The main purpose of heart valve centres as centres of excellence in the treatment of VHD is to deliver better quality of care. This is achieved through greater volumes associated with specialization of 


\section{Table 5 Recommended requirements of a heart valve centre (modified from Chambers et al. $^{32}$ )}

\section{Requirements}

Multidisciplinary teams with competencies in valve replacement, aortic root surgery, mitral, tricuspid and aortic valve repair, as well as transcatheter aortic and mitral valve techniques including reoperations and reinterventions. The Heart Teams must meet on a regular basis and work with standard operating procedures.

Imaging, including 3D and stress echocardiographic techniques, perioperative TOE, cardiac CT, MRI, and positron emission tomography-CT.

Regular consultation with community, other hospitals, and extracardiac departments, and between non-invasive cardiologists and surgeons and interventional cardiologists.

Back-up services including other cardiologists, cardiac surgeons, intensive care and other medical specialties.

\section{Data review:}

- Robust internal audit processes including mortality and

complications, repair rates, durability of repair, and reoperation rate with a minimum of I-year follow-up.

- Results available for review internally and externally.

- Participation in national or European quality databases.

$3 \mathrm{D}=$ three-dimensional; $\mathrm{CT}=$ computed tomography; $\mathrm{MRI}=$ magnetic resonance imaging; TOE = transoesophageal echocardiography training, continuing education and clinical interest. Specialization will also result in timely referral of patients before irreversible adverse effects occur and evaluation of complex VHD conditions. Techniques with a steep learning curve may be performed with better results in hospitals with high volumes and more experience. ${ }^{32}$ These main aspects are presented in Table 5.

A heart valve centre should have structured training programmes. ${ }^{32}$ Surgeons and cardiologists performing any valve intervention should undergo focused training as part of their basic local board certification training. Learning new techniques should take place through mentoring to minimize the effects of the 'learning curve'.

The relationship between case volume and outcomes for surgery and transcatheter interventions is complex but should not be denied. ${ }^{33-35}$ However, the precise numbers of procedures per individual operator or hospital required to provide high-quality care remain controversial and more scientific data are required before solid recommendations can be provided. Nevertheless, standards for provision of cardiac surgery that constitute the minimal core requirements have been released. ${ }^{36}$ Experience in the full spectrum of surgical procedures - including valve replacement; aortic root surgery; mitral, tricuspid and aortic valve repair; repair of complicated valve endocarditis such as root abscess; treatment of atrial fibrillation as well as surgical myocardial revascularization — must be available. The spectrum of interventional procedures in addition to TAVI should include mitral valvuloplasty, mitral valve repair (edge-to-edge), closure of atrial septal defects, closure of paravalvular leaks and left atrial (LA) appendage closure as well as percutaneous coronary intervention $(\mathrm{PCl})$. Expertise in interventional and surgical management of vascular diseases and complications must be available. Comprehensive recording of performance and patient outcome data at the level of the given heart valve centre is essential, as well as participation in national or ESC/EACTS registries.

\subsection{Management of associated conditions} 3.7.1 Coronary artery disease

The use of stress tests to detect CAD associated with severe valvular disease is discouraged because of their low diagnostic value and potential risks. A summary of the management of associated CAD is given in section 3.1.3.1 (see table of recommendations on the management of CAD in patients with VHD) and is detailed in specific guidelines. ${ }^{16}$

\subsubsection{Atrial fibrillation}

Non-vitamin K antagonist oral anticoagulants (NOACs) are approved only for non-valvular atrial fibrillation, but there is no uniform definition of this term. ${ }^{37}$ Recent subgroup analyses of randomized trials on atrial fibrillation support the use of rivaroxaban, apixaban, dabigatran and edoxaban in patients with aortic stenosis, aortic regurgitation or mitral regurgitation presenting with atrial fibrillation. ${ }^{38-41}$ The use of NOACs is discouraged in patients who have atrial fibrillation associated with moderate to severe mitral stenosis, given the lack of data and the particularly high thromboembolic risk. Despite the absence of data, NOACs may be used in patients who have atrial fibrillation associated with an aortic bioprosthesis $>3$ months after implantation but are strictly contraindicated in patients with any mechanical prostheses. ${ }^{42,43}$

Surgical ablation of atrial fibrillation combined with mitral valve surgery is effective in reducing the incidence of atrial fibrillation, but at the expense of more frequent pacemaker implantation, and has no impact on short-term survival. ${ }^{44}$ Surgical ablation should be considered in patients with symptomatic atrial fibrillation and may be considered in patients with asymptomatic atrial fibrillation if feasible with minimal risk. The decision should factor in other important variables, such as age, the duration of atrial fibrillation and LA size. Surgical excision or external clipping of the LA appendage may be considered combined with valvular surgery, although there is no evidence that it decreases thromboembolic risk. For patients with atrial fibrillation and risk factors for stroke, long-term oral anticoagulation is currently recommended, although surgical ablation of atrial fibrillation and/or surgical LA appendage excision or exclusion may have been performed. ${ }^{37}$ Recommendations for the management of atrial fibrillation in VHD are summarized in the following table. 


\section{Management of atrial fibrillation in patients with VHD}

\begin{tabular}{|c|c|c|}
\hline Recommendations & Class $^{\mathrm{a}}$ & Level $^{\mathbf{b}}$ \\
\hline \multicolumn{3}{|l|}{ Anticoagulation } \\
\hline $\begin{array}{l}\text { NOACs should be considered as an alterna- } \\
\text { tive to VKAs in patients with aortic stenosis, } \\
\text { aortic regurgitation and mitral regurgitation } \\
\text { presenting with atrial fibrillation. }{ }^{38-41}\end{array}$ & Ila & B \\
\hline $\begin{array}{l}\text { NOACs should be considered as an alterna- } \\
\text { tive to VKAs after the third month of } \\
\text { implantation in patients who have atrial } \\
\text { fibrillation associated with a surgical or } \\
\text { transcatheter aortic valve bioprosthesis. }\end{array}$ & Ila & C \\
\hline $\begin{array}{l}\text { The use of NOACs is not recommended in } \\
\text { patients with atrial fibrillation and moderate } \\
\text { to severe mitral stenosis. }\end{array}$ & III & C \\
\hline $\begin{array}{l}\text { NOACS are contraindicated in patients } \\
\text { with a mechanical valve. }{ }^{45}\end{array}$ & III & B \\
\hline \multicolumn{3}{|l|}{ Surgical interventions } \\
\hline $\begin{array}{l}\text { Surgical ablation of atrial fibrillation should } \\
\text { be considered in patients with symptomatic } \\
\text { atrial fibrillation who undergo valve } \\
\text { surgery. }{ }^{37}\end{array}$ & Ila & $\mathbf{A}$ \\
\hline $\begin{array}{l}\text { Surgical ablation of atrial fibrillation may be } \\
\text { considered in patients with asymptomatic } \\
\text { atrial fibrillation who undergo valve surgery, } \\
\text { if feasible, with minimal risk. }\end{array}$ & IIb & C \\
\hline $\begin{array}{l}\text { Surgical excision or external clipping of the } \\
\text { LA appendage may be considered in } \\
\text { patients undergoing valve surgery. }{ }^{46}\end{array}$ & IIb & B \\
\hline
\end{tabular}

$\mathrm{LA}=$ left atrial; NOAC = non-vitamin $\mathrm{K}$ antagonist oral anticoagulant; $\mathrm{VHD}=$ valvular heart disease; $\mathrm{VKA}=$ vitamin $\mathrm{K}$ antagonist.

${ }^{\mathrm{a}}$ Class of recommendation.

bLevel of evidence.

\section{Key points}

- Precise evaluation of the patient's history and symptomatic status as well as proper physical examination are crucial for the diagnosis and management of VHD.

- Echocardiography is the key technique to diagnose VHD and assess its severity and prognosis. Other non-invasive investigations such as stress testing, CMR, CT, fluoroscopy and biomarkers are complementary, and invasive investigation beyond preoperative coronary angiography is restricted to situations where non-invasive evaluation is inconclusive.

- Risk stratification is essential for decision making to weigh the risk of intervention against the expected natural history of VHD.

- Decision making in elderly patients requires special considerations, including life expectancy and expected quality of life, with regards to comorbidities and general condition (frailty).
- Heart valve centres with highly specialized multidisciplinary teams, comprehensive equipment and sufficient volumes of procedures are required to deliver high-quality care and provide adequate training.

- NOACs may be used in patients with atrial fibrillation and aortic stenosis, aortic regurgitation, mitral regurgitation or aortic bioprostheses $>3$ months after implantation but are contraindicated in mitral stenosis and mechanical valves.

\section{Gaps in evidence}

- Better tools for risk stratification need to be developed, particularly for the decision between surgery and catheter intervention and for the avoidance of futile interventions.

- Minimum volumes of procedures per operator and per hospital that are required to achieve optimal treatment results need to be defined.

- The safety and efficacy of NOACs in patients with surgical or transcatheter bioprostheses in the first 3 months after implantation should be studied.

\section{Aortic regurgitation}

Aortic regurgitation can be caused by primary disease of the aortic valve cusps and/or abnormalities of the aortic root and ascending aortic geometry. Degenerative tricuspid and bicuspid aortic regurgitation are the most common aetiologies in Western countries, accounting for approximately two-thirds of the underlying aetiology of aortic regurgitation in the Euro Heart Survey on VHD. ${ }^{47}$ Other causes include infective and rheumatic endocarditis. Acute severe aortic regurgitation is mostly caused by infective endocarditis and less frequently by aortic dissection.

\subsection{Evaluation}

\subsubsection{Echocardiography}

Echocardiography (TTE/TOE) is the key examination to describe valve anatomy, quantify aortic regurgitation, evaluate its mechanisms, define the morphology of the aorta and determine the feasibility of valve-sparing aortic surgery or valve repair. ${ }^{48,49}$

\section{Essential aspects of this evaluation include}

- Assessment of valve morphology: tricuspid, bicuspid, unicuspid or quadricuspid valve.

- Determination of the direction of the aortic regurgitation jet in the long-axis view (central or eccentric) and its origin in the short-axis view (central or commissural).

- Identification of the mechanism, following the same principle as for mitral regurgitation: normal cusps but insufficient coaptation due to dilatation of the aortic root with central jet (type 1), cusp prolapse with eccentric jet (type 2) or retraction with poor cusp tissue quality and large central or eccentric jet (type 3). ${ }^{48}$

- Quantification of aortic regurgitation should follow an integrated approach considering all qualitative, semi-quantitative and quantitative parameters 2,6 (Table 4).

- Measurement of LV function and dimensions. Indexing LV diameters for body surface area (BSA) is recommended in patients with small body size $\left(B S A<1.68 \mathrm{~m}^{2}\right){ }^{50}$ New parameters 
obtained by three-dimensional (3D) echocardiography, tissue Doppler and strain rate imaging may be useful, particularly in patients with borderline left ventricular ejection fraction (LVEF), where they may help in the decision for surgery. ${ }^{51}$

- Measurement of the aortic root and ascending aorta in the 2dimensional (2D) mode at four levels: annulus, sinuses of Valsalva, sinotubular junction and tubular ascending aorta. ${ }^{52}$ Measurements are taken in the parasternal long-axis view from leading edge to leading edge at end diastole, except for the aortic annulus, which is measured in mid systole. As it will have surgical consequences, it is important to differentiate three phenotypes of the ascending aorta: aortic root aneurysms (sinuses of Valsalva $>45 \mathrm{~mm}$ ), tubular ascending aneurysm (sinuses of Valsalva $<40$ $45 \mathrm{~mm}$ ) and isolated aortic regurgitation (all diameters $<40 \mathrm{~mm}$ ). The calculation of indexed values has been recommended to account for body size. ${ }^{53}$

- Definition of the anatomy of the aortic valve cusps and assessment of valve reparability should be provided by preoperative TOE if aortic valve repair or a valve-sparing surgery of the aortic root is considered.

- Intraoperative evaluation of the surgical result by TOE is mandatory in patients in whom the aortic valve is preserved or repaired in the procedure.

\subsubsection{Computed tomography and cardiac magnetic resonance}

CMR should be used to quantify the regurgitant fraction when echocardiographic measurements are equivocal. In patients with aortic dilatation, gated MSCT is recommended to assess the maximum diameter. CMR can be used for follow-up, but indication for surgery should preferably be based on CT measurements. Different methods of aortic measurements have been reported and this may result in diameter discrepancies of $2-3 \mathrm{~mm}$ that could influence therapeutic management. To improve reproducibility, it is recommended to measure diameters using the inner-inner edge technique at end diastole on the strictly transverse plane by double oblique reconstruction perpendicular to the axis of blood flow of the corresponding segment. Diameters at the annulus, sinus of Valsalva, sinotubular junction, tubular ascending aorta and aortic arch level should be reported. Maximum root diameter should be taken from sinus to sinus rather than sinus to commissure diameter, as it correlates more closely to long-axis leading edge to leading edge echo maximum diameters. ${ }^{54,55}$

\subsection{Indications for intervention}

Acute aortic regurgitation may require urgent surgery. It is primarily caused by infective endocarditis and aortic dissections. Specific guidelines deal with these entities. ${ }^{28,56}$ The indications for intervention in chronic aortic regurgitation are summarized on the next page (recommendations on indications for surgery in severe aortic regurgitation and aortic root disease) and in Figure 1 and may be related to symptoms, status of the LV or dilatation of the aorta.

In symptomatic patients, surgery is recommended irrespective of the LVEF value, except for extreme cases, as long as aortic regurgitation is severe and the operative risk is not prohibitive. ${ }^{57}$

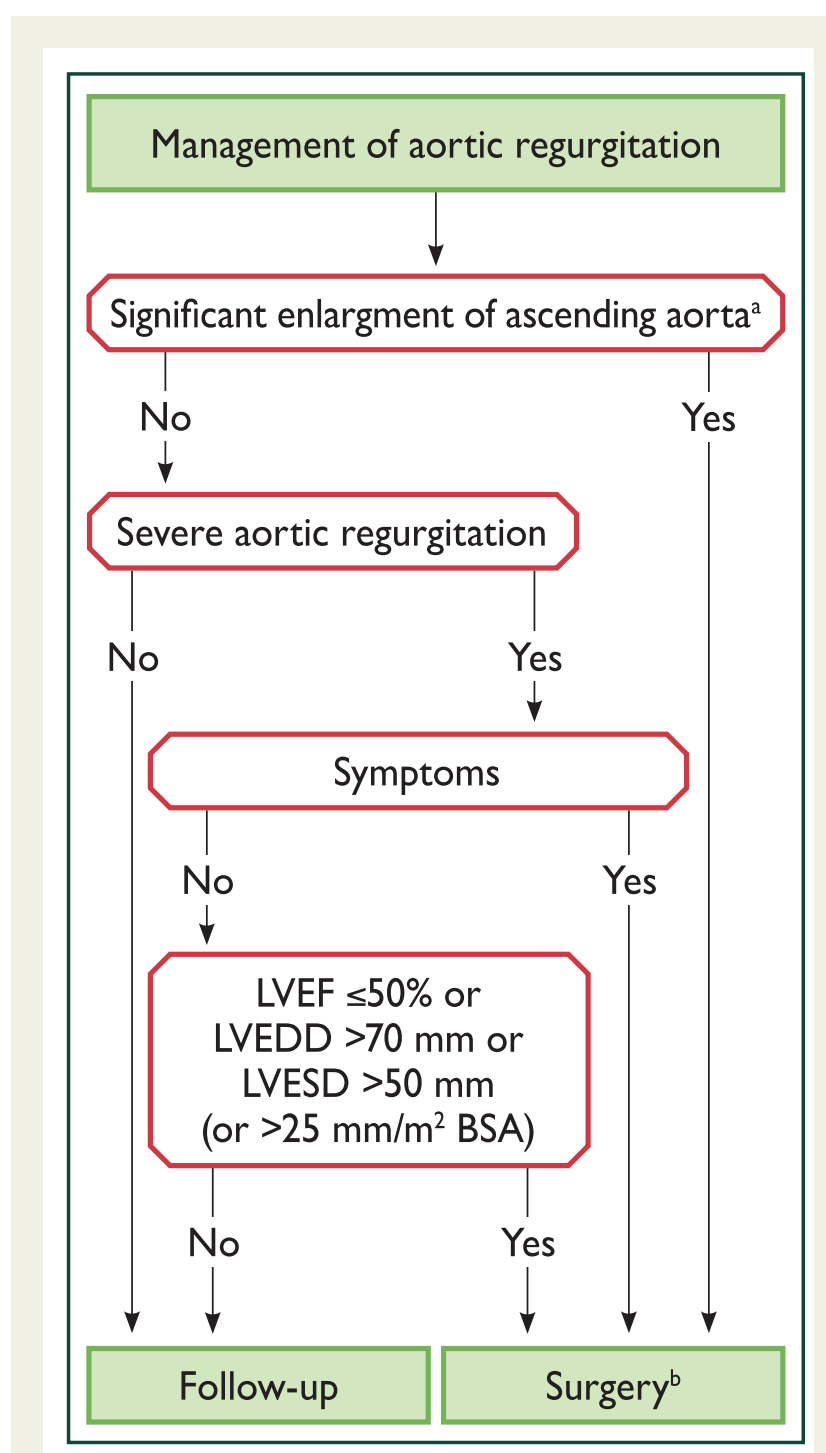

Figure I Management of aortic regurgitation. $A R=$ aortic regurgitation; BSA = body surface area; LVEDD = left ventricle end-diastolic diameter; LVEF = left ventricular ejection fraction; LVESD = left ventricle end-systolic diameter.

${ }^{a}$ See table of recommendations on indications for surgery in severe aortic regurgitation and aortic root disease for definition.

bSurgery should also be considered if significant changes in LV or aortic size occur during follow-up (see table of recommendations on indications for surgery in severe aortic regurgitation and aortic root disease in section 4.2).

In asymptomatic patients with severe aortic regurgitation, impairment of LV function (ejection fraction $\leq 50 \%$ ) and LV enlargement with an LV end-diastolic diameter (LVEDD) $>70 \mathrm{~mm}$ or left ventricular end-systolic diameter (LVESD) $>50 \mathrm{~mm}$ are associated with 
worse outcome and surgery should therefore be pursued when these cut-offs are reached. ${ }^{58}$ In patients with small body size, LVESD should be related to BSA and a cut-off of $25 \mathrm{~mm} / \mathrm{m}^{2}$ BSA appears to be more appropriate. ${ }^{50}$ In patients not reaching the thresholds for surgery, close follow-up is needed and exercise testing should be performed to identify borderline symptomatic patients. In truly asymptomatic patients, regular assessment of LV function and physical condition are crucial to identify the optimal time for surgery. A rapid progression of ventricular dimensions or decline in ventricular function on serial testing is a reason to consider surgery.

In patients with a dilated aorta, the rationale for surgery has been best defined in patients with Marfan syndrome and root dilation. ${ }^{59}$ Root aneurysms need to have root replacement, with or without preservation of the native aortic valve, but definitely with coronary reimplantation. In contrast, tubular ascending aortic aneurysms require only a supracommissural tube graft replacement without coronary reimplantation. In patients with aortic diameters borderline for aortic surgery, the family history, age and anticipated risk of the procedure should be taken into consideration. In individuals with a bicuspid aortic valve and no significant valve regurgitation, prophylactic surgery should be considered with aortic diameters $\geq 55 \mathrm{~mm}$ or $\geq 50 \mathrm{~mm}$ when additional risk factors or coarctation are present (see table of recommendations on indications for surgery in severe aortic regurgitation and aortic root disease). Surgery is indicated in all patients with Marfan syndrome and a maximal aortic diameter $\geq 50 \mathrm{~mm}$. In patients with Marfan syndrome and additional risk factors and in patients with a TGFBR1 or TGFBR2 mutation (including Loeys-Dietz syndrome), surgery should be considered at a maximal aortic diameter $\geq 45 \mathrm{~mm} .{ }^{60}$ In the latter group, women with low BSA, patients with a TGFBR2 mutation or patients with severe extra-aortic features appear to be at particularly high risk and surgery may be considered already at a lower threshold of $40 \mathrm{~mm}^{60}$ In aortic roots $\geq 55 \mathrm{~mm}$, surgery should be considered irrespective of the degree of aortic regurgitation and type of valve pathology. ${ }^{61}$ For patients who have an indication for aortic valve surgery, an aortic diameter $\geq 45 \mathrm{~mm}$ is considered to indicate concomitant surgery of the aortic root or tubular ascending aorta. The patient's stature, the aetiology of the valvular disease (bicuspid valve) and the intraoperative shape and wall thickness of the ascending aorta should be taken into account for individual decisions.

Although valve replacement is the standard procedure in the majority of patients with aortic regurgitation, valve repair or valvesparing surgery should be considered in patients with pliable noncalcified tricuspid or bicuspid valves who have a type I (enlargement of the aortic root with normal cusp motion) or type II (cusp prolapse) mechanism of aortic regurgitation. ${ }^{6,48,49}$ In experienced centres, valve-sparing root replacement and valve repair, when feasible, yield good long-term results with low rates of valve-related events as well as better quality of life. ${ }^{62-65}$ The choice of the surgical procedure should be adapted to the experience of the team, the presence of an aortic root aneurysm, characteristics of the cusps, life expectancy and desired anticoagulation status. Patients in whom the Heart Team identifies the aortic valve to be repairable should be referred to appropriate surgical teams for the procedure.
Indications for surgery in (A) severe aortic regurgitation and (B) aortic root disease (irrespective of the severity of aortic regurgitation)

\begin{tabular}{|c|c|c|}
\hline Indications for surgery & Class $^{\mathrm{a}}$ & Level $^{\mathrm{b}}$ \\
\hline \multicolumn{3}{|l|}{ A. Severe aortic regurgitation } \\
\hline Surgery is indicated in symptomatic patients. ${ }^{57,58,66,67}$ & I & B \\
\hline $\begin{array}{l}\text { Surgery is indicated in asymptomatic patients with resting } \\
\text { LVEF } \leq 50 \% .^{57.58}\end{array}$ & $\mathbf{I}$ & B \\
\hline $\begin{array}{l}\text { Surgery is indicated in patients undergoing CABG or sur- } \\
\text { gery of the ascending aorta or of another valve. }\end{array}$ & 1 & C \\
\hline $\begin{array}{l}\text { Heart Team discussion is recommended in selected } \\
\text { patients }{ }^{c} \text { in whom aortic valve repair may be a feasible } \\
\text { alternative to valve replacement. }\end{array}$ & 1 & C \\
\hline $\begin{array}{l}\text { Surgery should be considered in asymptomatic patients } \\
\text { with resting ejection fraction }>50 \% \text { with severe LV dilata- } \\
\text { tion: } \text { LVEDD }>70 \mathrm{~mm} \text { or LVESD }>50 \mathrm{~mm} \text { (or LVESD } \\
>25 \mathrm{~mm} / \mathrm{m}^{2} \text { BSA in patients with small body size). }{ }^{58,66}\end{array}$ & Ila & B \\
\hline \multicolumn{3}{|c|}{$\begin{array}{l}\text { B. Aortic root or tubular ascending aortic aneurysm }{ }^{d} \text { (irrespective of the } \\
\text { severity of aortic regurgitation) }\end{array}$} \\
\hline $\begin{array}{l}\text { Aortic valve repair, using the reimplantation or remodel- } \\
\text { ling with aortic annuloplasty technique, is recommended in } \\
\text { young patients with aortic root dilation and tricuspid aortic } \\
\text { valves, when performed by experienced surgeons. }\end{array}$ & I & C \\
\hline $\begin{array}{l}\text { Surgery is indicated in patients with Marfan syndrome who } \\
\text { have aortic root disease with a maximal ascending aortic } \\
\text { diameter } \geq 50 \mathrm{~mm} \text {. }\end{array}$ & I & C \\
\hline \multirow{4}{*}{$\begin{array}{l}\text { Surgery should be considered in patients who have aortic } \\
\text { root disease with maximal ascending aortic diameter: } \\
\text { - } \geq 45 \mathrm{~mm} \text { in the presence of Marfan syndrome and } \\
\text { additional risk factors }{ }^{\mathrm{e}} \text { or patients with a TGFBR1 or } \\
\text { TGFBR2 mutation (including Loeys-Dietz syndrome). } \\
\text { - } \geq 50 \mathrm{~mm} \text { in the presence of a bicuspid valve with } \\
\text { additional risk factors }{ }^{\mathrm{e}} \text { or coarctation. } \\
\text { - } \geq 55 \mathrm{~mm} \text { for all other patients. }\end{array}$} & IIa & C \\
\hline & Ila & C \\
\hline & Ila & C \\
\hline & Ila & c \\
\hline $\begin{array}{l}\text { When surgery is primarily indicated for the aortic valve, } \\
\text { replacement of the aortic root or tubular ascending aorta } \\
\text { should be considered when } \geq 45 \mathrm{~mm} \text {, particularly in the } \\
\text { presence of a bicuspid valve. }\end{array}$ & Ila & C \\
\hline
\end{tabular}

$\mathrm{BSA}=$ body surface area; $\mathrm{CABG}=$ coronary artery bypass grafting; $\mathrm{CT}=\mathrm{com}$ puted tomography; ECG = electrocardiogram; LV = left ventricular; LVEDD = left ventricular end-diastolic diameter; LVEF = left ventricular ejection fraction; LVESD = left ventricular end-systolic diameter.

${ }^{\mathrm{a}}$ Class of recommendation.

'Level of evidence.

'Patients with pliable non-calcified tricuspid or bicuspid valves who have a type I (enlargement of the aortic root with normal cusp motion) or type II (cusp prolapse) mechanism of aortic regurgitation. ${ }^{6,48,49}$

${ }^{d}$ For clinical decision making, dimensions of the aorta should be confirmed by ECG-gated CT measurement.

${ }^{\mathrm{e}}$ Family history of aortic dissection (or personal history of spontaneous vascular dissection), severe aortic regurgitation or mitral regurgitation, desire for pregnancy, systemic hypertension and/or aortic size increase $>3 \mathrm{~mm} / \mathrm{year}$ (on repeated measurements using the same ECG-gated imaging technique measured at the same level of the aorta with side-by-side comparison and confirmed by another technique).

${ }^{\mathrm{f}} \mathrm{A}$ lower threshold of $40 \mathrm{~mm}$ may be considered in women with low BSA, in patients with a TGFBR2 mutation or in patients with severe extra-aortic features. $^{60}$

${ }^{8}$ Considering age, BSA, aetiology of the valvular disease, presence of a bicuspid aortic valve and intraoperative shape and thickness of the ascending aorta. 


\subsection{Medical therapy}

Medical therapy can provide symptomatic improvement in individuals with chronic severe aortic regurgitation in whom surgery is not feasible. In patients who undergo surgery but continue to suffer from heart failure or hypertension, angiotensin-converting enzyme (ACE) inhibitors, angiotensin receptor blockers (ARBs) and beta-blockers are useful. $^{68,69}$

In patients with Marfan syndrome, beta-blockers and/or losartan may slow aortic root dilatation and reduce the risk of aortic complications and should be considered before and after surgery. ${ }^{70-72}$ By analogy, while there are no studies that provide evidence, it is common clinical practice to advise beta-blocker or losartan therapy in patients with bicuspid aortic valve if the aortic root and/or ascending aorta is dilated.

Women with Marfan syndrome and an aortic diameter $>45 \mathrm{~mm}$ are strongly discouraged from becoming pregnant without prior repair because of the high risk of dissection. Although an aortic diameter $<40 \mathrm{~mm}$ is rarely associated with aortic dissection, a completely safe diameter does not exist. With an aorta between 40 and $45 \mathrm{~mm}$, previous aortic growth and family history are important for advising pregnancy with or without aortic repair. ${ }^{73}$ Although the actual risk of dissection is not well-documented in the setting of bicuspid valves, counselling against pregnancy is recommended in the setting of aortic diameters $>50 \mathrm{~mm}^{74}$

The level of physical and sports activity in the presence of a dilated aorta remains a matter of clinical judgement in the absence of evidence. Current guidelines are very restrictive, particularly regarding isometric exercise, to avoid a catastrophic event. ${ }^{75}$ This attitude is clearly justified in the presence of connective tissue disease.

Given the family risk of thoracic aortic aneurysms, screening and referral for genetic testing of the patient's first-degree relatives with appropriate imaging studies is indicated in patients with connective tissue disease. For patients with bicuspid valves it is appropriate to have an echocardiographic screening of first-degree relatives.

\subsection{Serial testing}

All asymptomatic patients with severe aortic regurgitation and normal LV function should be seen for follow-up at least every year. In patients with a first diagnosis, or if LV diameter and/or ejection fraction show significant changes or come close to thresholds for surgery, follow-up should be continued at 3-6-month intervals. In inconclusive cases, BNP may be helpful, as its elevation during followup has been related to deterioration of LV function. ${ }^{76}$ Patients with mild to moderate aortic regurgitation can be reviewed on a yearly basis and echocardiography performed every 2 years.

If the ascending aorta is dilated $(>40 \mathrm{~mm})$ it is recommended to perform CT or CMR. Follow-up assessment of the aortic dimension should be performed using echocardiography and/or CMR. Any increase $>3 \mathrm{~mm}$ should be validated by $\mathrm{CT}$ angiography/CMR and compared to baseline data.

\subsection{Special patient populations}

If aortic regurgitation requiring surgery is associated with severe mitral regurgitation, both should be addressed during the same operation.
In patients with moderate aortic regurgitation who undergo coronary artery bypass grafting (CABG) or mitral valve surgery, the decision to treat the aortic valve is controversial, as data show that progression of moderate aortic regurgitation is very slow in patients without aortic dilatation. ${ }^{77}$ The Heart Team should decide based on the aetiology of aortic regurgitation, other clinical factors, the life expectancy of the patient and the patient's operative risk.

\section{Key points}

- The evaluation of aortic regurgitation requires consideration of valve morphology and the mechanism and severity of regurgitation, including careful assessment of aortic dilatation.

- In asymptomatic patients with severe aortic regurgitation, careful follow-up of symptomatic status and LV size and function is mandatory.

- The strongest indication for valve surgery is the presence of symptoms (spontaneous or on exercise testing) and/or the documentation of LVEF $<50 \%$ and/or end-systolic diameter $>50 \mathrm{~mm}$.

- In patients with a dilated aorta, definition of the aortic pathology and accurate measurements of aortic diameters are crucial to guide the timing and type of surgery.

- Aortic valve repair and valve-sparing aortic surgery instead of aortic valve replacement should be considered in selected cases in experienced centres.

\section{Gaps in evidence}

- The impact of earlier markers of LV dysfunction on postoperative outcome requires further research.

- Criteria for the decision between valve replacement and valve repair must still be refined.

- Potential differences in the risk of aortic complications depending on subtypes of aortic aneurysms (site and morphology) should be studied.

- The effect of medical treatment on aortic enlargement in patients with bicuspid aortic valve needs to be studied.

\section{Aortic stenosis}

Aortic stenosis is the most common primary valve disease leading to surgery or catheter intervention in Europe and North America, with a growing prevalence due to the ageing population.

\subsection{Evaluation}

\subsubsection{Echocardiography}

Echocardiography is the key diagnostic tool. It confirms the presence of aortic stenosis; assesses the degree of valve calcification, LV function and wall thickness; detects the presence of other associated valve disease or aortic pathology and provides prognostic information. Doppler echocardiography is the preferred technique for assessing the severity of aortic stenosis. ${ }^{4}$

Figure 2 and Table 6 provide a practical stepwise approach for the assessment of aortic stenosis severity. Details can be found in a recent position paper from the European Association of Cardiovascular Imaging. ${ }^{4}$

Although valve area represents, from a theoretical perspective, the ideal measurement for assessing the severity of aortic stenosis, it has 


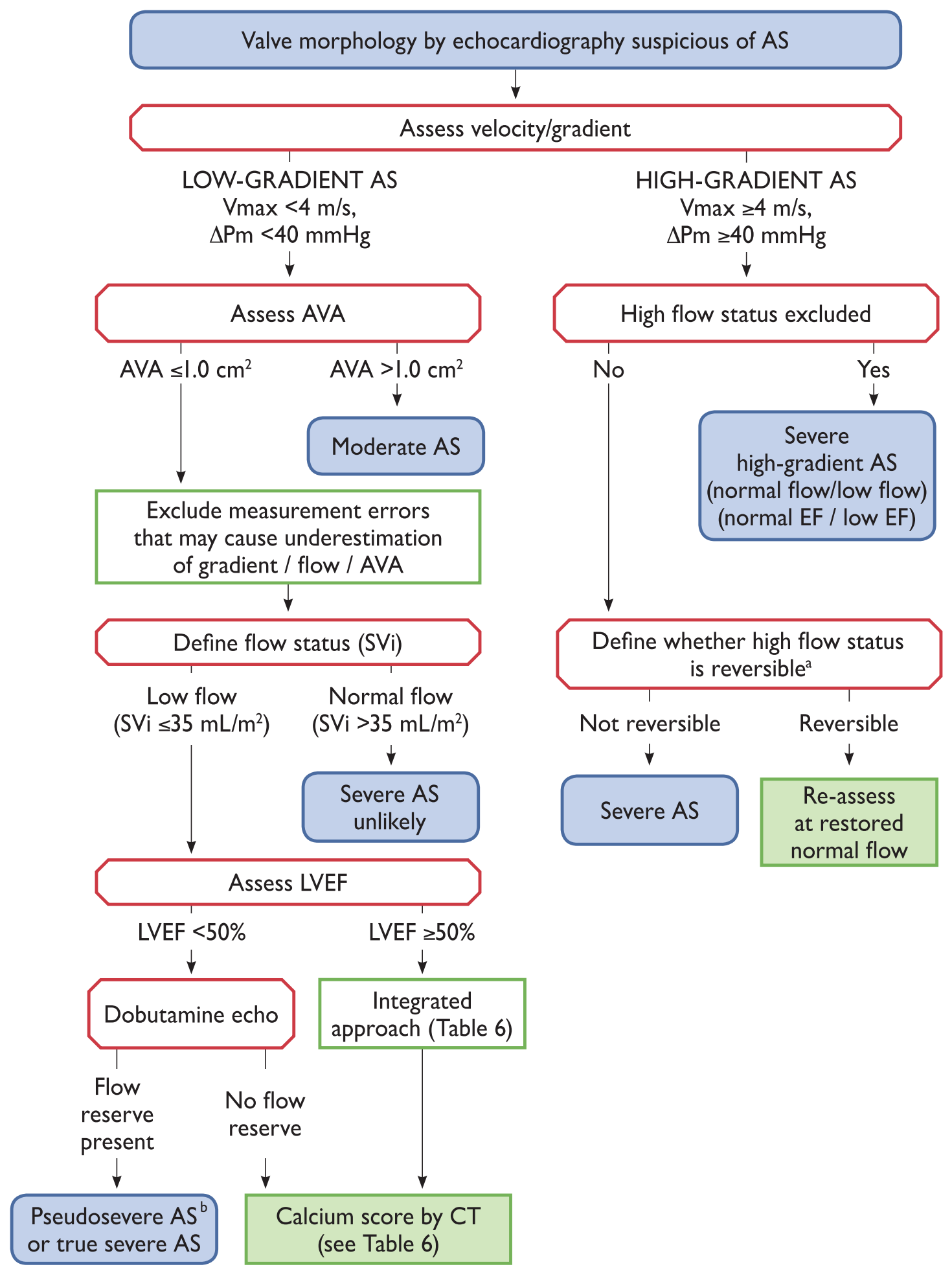

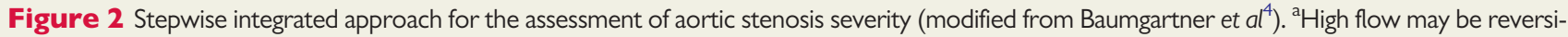

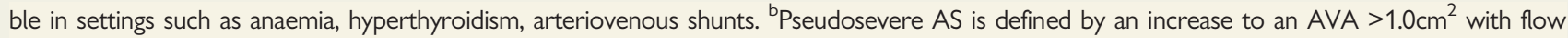
normalization.

$\Delta \mathrm{Pm}=$ mean transvalvular pressure gradient; $\mathrm{AS}=$ aortic stenosis; $\mathrm{AVA}=$ aortic valve area; $\mathrm{CT}=$ computed tomography; $\mathrm{EF}=$ ejection fraction; $\mathrm{LVEF}=$ left ventricular ejection fraction; $\mathrm{SVi}$ = stroke volume index; $V$ max = peak transvalvular velocity. 
Table 6 Criteria that increase the likelihood of severe aortic stenosis in patients with AVA $<1.0 \mathrm{~cm}^{2}$ and mean gradient $<40 \mathrm{mmHg}$ in the presence of preserved ejection fraction (modified from Baumgartner et al. ${ }^{4}$ )

\begin{tabular}{|c|c|}
\hline \multicolumn{2}{|l|}{ Criteria } \\
\hline Clinical criteria & $\begin{array}{l}\text { - Typical symptoms without other explanation } \\
\text { - Elderly patient ( }>70 \text { years) }\end{array}$ \\
\hline Qualitative imaging data & $\begin{array}{l}\text { - LV hypertrophy (additional history of hypertension to be considered) } \\
\text { - Reduced LV longitudinal function without other explanation }\end{array}$ \\
\hline \multirow[t]{3}{*}{ Quantitative imaging data } & - Mean gradient $30-40 \mathrm{mmHg}^{\mathrm{a}}$ \\
\hline & $\begin{array}{l}\text { - Low flow }\left(\mathrm{SVi}<35 \mathrm{~mL} / \mathrm{m}^{2}\right) \text { confirmed by techniques other than standard Doppler technique } \\
\text { (LVOT measurement by 3D TOE or MSCT; CMR, invasive data) }\end{array}$ \\
\hline & $\begin{array}{l}\text { - Calcium score by } \text { MSCT }^{\text {b }} \\
\text { Severe aortic stenosis very likely: men } \geq 3000 \text {; women } \geq 1600 \\
\text { Severe aortic stenosis likely: men } \geq 2000 \text {; women } \geq 1200 \\
\text { Severe aortic stenosis unlikely: men }<1600 \text {; women }<800\end{array}$ \\
\hline
\end{tabular}

$3 \mathrm{D}=$ three-dimensional; $\mathrm{AVA}=$ aortic valve area; $\mathrm{CMR}=$ cardiovascular magnetic resonance; $\mathrm{LV}=$ left ventricular; $\mathrm{LVOT}=$ left ventricular outflow tract; MSCT = multislice computed tomography; $\mathrm{SVi}$ = stroke volume index; $\mathrm{TOE}=$ transoesophageal echocardiography.

${ }^{a}$ Haemodynamics measured when the patient is normotensive.

bValues are given in arbitrary units using Agatston method for quantification of valve calcification.

technical limitations in clinical practice. It must, for clinical decision making, always be considered together with flow rate, mean pressure gradient (the most robust measurement), ventricular function, size and wall thickness, degree of valve calcification, blood pressure and functional status. Hypertensive patients should be reassessed when normotensive. ${ }^{4}$ Four categories of aortic stenosis can be defined:

- High-gradient aortic stenosis (valve area $<1 \mathrm{~cm}^{2}$, mean gradient $>40 \mathrm{mmHg}$ ). Severe aortic stenosis can be assumed irrespective of whether LVEF and flow are normal or reduced.

- Low-flow, low-gradient aortic stenosis with reduced ejection fraction [valve area $<1 \mathrm{~cm}^{2}$, mean gradient $<40 \mathrm{mmHg}$, ejection fraction $<50 \%$, stroke volume index $\left.(\mathrm{SVi}) \leq 35 \mathrm{~mL} / \mathrm{m}^{2}\right]$. Low-dose dobutamine echocardiography is recommended in this setting to distinguish truly severe aortic stenosis from pseudosevere aortic stenosis, which is defined by an increase to an aortic valve area (AVA) of $>1.0 \mathrm{~cm}^{2}$ with flow normalization. In addition, the presence of flow reserve (also termed contractile reserve; increase of stroke volume $>20 \%$ ) has prognostic implications because it is associated with better outcome. ${ }^{10,78}$

- Low-flow, low-gradient aortic stenosis with preserved ejection fraction (valve area $<1 \mathrm{~cm}^{2}$, mean gradient $<40 \mathrm{mmH}$, ejection fraction $\geq 50 \%, \mathrm{SVi} \leq 35 \mathrm{~mL} / \mathrm{m}^{2}$ ). This is typically encountered in the elderly and is associated with small ventricular size, marked LV hypertrophy and frequently a history of hypertension. ${ }^{79,80}$ The diagnosis of severe aortic stenosis in this setting remains challenging and requires careful exclusion of measurement errors and other reasons for such echocardiographic findings (Table 6). The degree of valve calcification by MSCT is related to aortic stenosis severity and outcome. ${ }^{13,14,81}$ Its assessment has therefore gained increasing importance in this setting.

- Normal-flow, low-gradient aortic stenosis with preserved ejection fraction (valve area $<1 \mathrm{~cm}^{2}$, mean gradient $<40 \mathrm{mmH}$, ejection fraction $\geq 50 \%, \mathrm{SVi}>35 \mathrm{~mL} / \mathrm{m}^{2}$ ). These patients will in general have only moderate aortic stenosis. ${ }^{14,82-84}$
5.1.2 Additional diagnostic aspects, including assessment of prognostic parameters

Exercise testing is recommended in physically active patients for unmasking symptoms and for risk stratification of asymptomatic patients with severe aortic stenosis. ${ }^{85}$

Exercise stress echocardiography may provide prognostic information in asymptomatic severe aortic stenosis by assessing the increase in mean pressure gradient and change in LV function during exercise. $^{86}$

TOE provides additional evaluation of concomitant mitral valve abnormalities. It has gained importance in the assessment before TAVI and after TAVI or surgical procedures. ${ }^{87}$

MSCT and CMR provide additional information on the dimensions and geometry of the aortic root and ascending aorta and the extent of calcification. It has become particularly important for the quantification of valve calcification when assessing aortic stenosis severity in low-gradient aortic stenosis. ${ }^{13,14,81}$ CMR may be useful for the detection and quantification of myocardial fibrosis, providing additional prognostic information regardless of the presence of CAD. ${ }^{88}$

Natriuretic peptides have been shown to predict symptom-free survival and outcome in normal and low-flow severe aortic stenosis $^{89,90}$ and may be useful in asymptomatic patients to determine optimal timing of intervention.

Retrograde LV catheterization to assess the severity of aortic stenosis is no longer routinely performed. Its use is restricted to patients with inconclusive non-invasive investigations.

\subsubsection{Diagnostic workup before transcatheter aortic valve implantation}

MSCT is the preferred imaging tool to assess the anatomy and dimensions of the aortic root, size and shape of the aortic valve annulus, its distance to the coronary ostia, the distribution of calcifications and 
the number of aortic valve cusps. It is essential to evaluate the feasibility of the various access routes, as this provides information on minimal luminal diameters, atherosclerotic plaque burden, the presence of aneurysms or thrombi, vessel tortuosity and thoracic and LV apex anatomy. CMR — as an alternative technique — is, in this context, inferior to MSCT with regards to assessment of inner vessel dimensions and calcifications. 3D TOE can be used to determine aortic annulus dimensions but remains more operator- and image quality-dependent than MSCT. However, TOE is an important tool for monitoring the procedure and evaluating the results, especially if complications occur.

\subsection{Indications for intervention}

The indications for aortic valve interventions are summarized on the next page (see table of indications for intervention in aortic stenosis and recommendations for the choice of intervention mode) and in Table 7 and are illustrated in Figure 3.

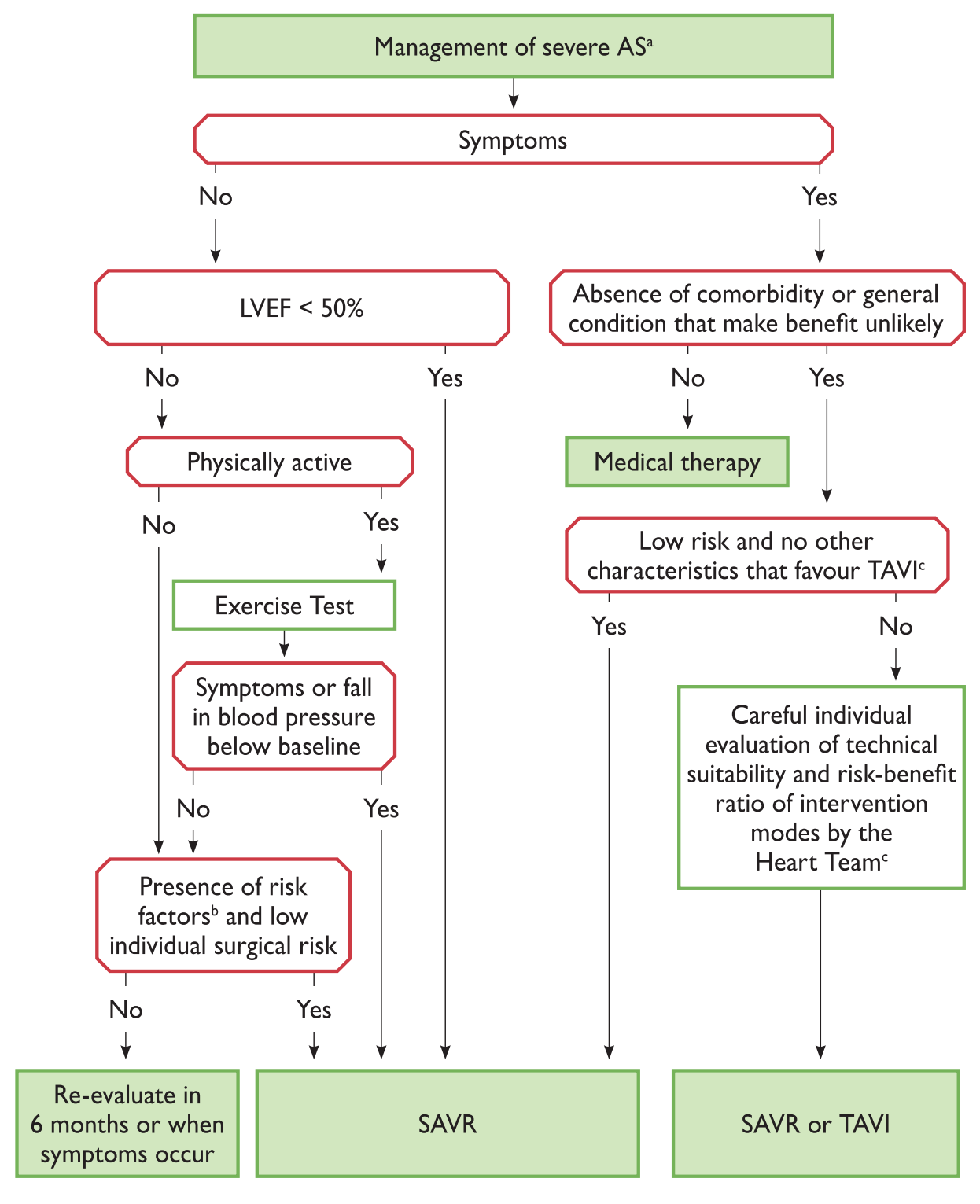

Figure 3 Management of severe aortic stenosis. AS = aortic stenosis; LVEF = left ventricular ejection fraction; SAVR = surgical aortic valve replacement; TAVI = transcatheter aortic valve implantation.

${ }^{a}$ See Figure 2 and Table 6 for the definition of severe AS.

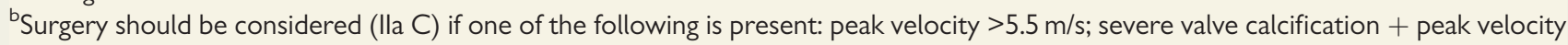
progression $\geq 0.3 \mathrm{~m} / \mathrm{s}$ per year; markedly elevated neurohormones (>threefold age- and sex-corrected normal range) without other explanation; severe pulmonary hypertension (systolic pulmonary artery pressure $>60 \mathrm{mmHg}$ ).

'See Table 7 and Table of Recommendations in section 5.2 Indications for interventions in aortic stenosis. 
Indications for intervention in aortic stenosis and recommendations for the choice of intervention mode

\begin{tabular}{|c|c|c|}
\hline A) Symptomatic aortic stenosis & Class $^{\mathbf{a}}$ & Level $^{\mathbf{b}}$ \\
\hline $\begin{array}{l}\text { Intervention is indicated in symptomatic patients with severe, high-gradient aortic stenosis (mean gradient } \geq 40 \text { mmHg or peak velocity } \\
\geq 4.0 \mathrm{~m} / \mathrm{s}){ }^{91-93}\end{array}$ & $\mathbf{I}$ & $\mathbf{B}$ \\
\hline $\begin{array}{l}\text { Intervention is indicated in symptomatic patients with severe low-flow, low-gradient }(<40 \mathrm{mmHg} \text { ) aortic stenosis with reduced ejection frac- } \\
\text { tion and evidence of flow (contractile) reserve excluding pseudosevere aortic stenosis. }\end{array}$ & $\mathbf{I}$ & C \\
\hline $\begin{array}{l}\text { Intervention should be considered in symptomatic patients with low-flow, low-gradient }(<40 \mathrm{mmHg} \text { ) aortic stenosis with normal ejection } \\
\left.\text { fraction after careful confirmation of severe aortic stenosis }{ }^{c} \text { (see Figure } 2 \text { and Table } 6\right) \text {. }\end{array}$ & Illa & C \\
\hline $\begin{array}{l}\text { Intervention should be considered in symptomatic patients with low-flow, low-gradient aortic stenosis and reduced ejection fraction without } \\
\text { flow (contractile) reserve, particularly when CT calcium scoring confirms severe aortic stenosis. }\end{array}$ & Ila & C \\
\hline $\begin{array}{l}\text { Intervention should not be performed in patients with severe comorbidities when the intervention is unlikely to improve quality of life or } \\
\text { survival. }\end{array}$ & III & C \\
\hline \multicolumn{3}{|l|}{ B) Choice of intervention in symptomatic aortic stenosis } \\
\hline $\begin{array}{l}\text { Aortic valve interventions should only be performed in centres with both departments of cardiology and cardiac surgery on site and with } \\
\text { structured collaboration between the two, including a Heart Team (heart valve centres). }\end{array}$ & $\mathbf{I}$ & C \\
\hline $\begin{array}{l}\text { The choice for intervention must be based on careful individual evaluation of technical suitability and weighing of risks and benefits of each } \\
\text { modality (aspects to be considered are listed in Table 7). In addition, the local expertise and outcomes data for the given intervention must } \\
\text { be taken into account. }\end{array}$ & $\mathbf{I}$ & C \\
\hline $\begin{array}{l}\text { SAVR is recommended in patients at low surgical risk (STS or EuroSCORE } \|<4 \% \text { or logistic EuroSCORE I }<10 \%{ }^{\mathrm{d}} \text { and no other risk factors } \\
\text { not included in these scores, such as frailty, porcelain aorta, sequelae of chest radiation). }{ }^{93}\end{array}$ & $\mathbf{I}$ & $\mathbf{B}$ \\
\hline TAVI is recommended in patients who are not suitable for SAVR as assessed by the Heart Team. ${ }^{91,94}$ & $\mathbf{I}$ & B \\
\hline $\begin{array}{l}\text { In patients who are at increased surgical risk (STS or EuroSCORE II } \geq 4 \% \text { or logistic EuroSCORE I } \geq 10 \%{ }^{\mathrm{d}} \text { or other risk factors not included } \\
\text { in these scores such as frailty, porcelain aorta, sequelae of chest radiation), the decision between SAVR and TAVI should be made by the } \\
\text { Heart Team according to the individual patient characteristics (see Table 7), with TAVI being favoured in elderly patients suitable for transfe- } \\
\text { moral access. }{ }^{91,94-102}\end{array}$ & $\mathbf{I}$ & $\mathbf{B}$ \\
\hline $\begin{array}{l}\text { Balloon aortic valvotomy may be considered as a bridge to SAVR or TAVI in haemodynamically unstable patients or in patients with sympto- } \\
\text { matic severe aortic stenosis who require urgent major non-cardiac surgery. }\end{array}$ & Illb & C \\
\hline $\begin{array}{l}\text { Balloon aortic valvotomy may be considered as a diagnostic means in patients with severe aortic stenosis or other potential causes for symp- } \\
\text { toms (i.e. lung disease) and in patients with severe myocardial dysfunction, pre-renal insufficiency or other organ dysfunction that may be } \\
\text { reversible with balloon aortic valvotomy when performed in centres that can escalate to TAVI. }\end{array}$ & Illb & C \\
\hline \multicolumn{3}{|l|}{ C) Asymptomatic patients with severe aortic stenosis (refers only to patients eligible for surgical valve replacement) } \\
\hline SAVR is indicated in asymptomatic patients with severe aortic stenosis and systolic LV dysfunction (LVEF $<50 \%)$ not due to another cause. & I & C \\
\hline $\begin{array}{l}\text { SAVR is indicated in asymptomatic patients with severe aortic stenosis and an abnormal exercise test showing symptoms on exercise clearly } \\
\text { related to aortic stenosis. }\end{array}$ & $\mathbf{I}$ & C \\
\hline $\begin{array}{l}\text { SAVR should be considered in asymptomatic patients with severe aortic stenosis and an abnormal exercise test showing a decrease in blood } \\
\text { pressure below baseline. }\end{array}$ & Ila & C \\
\hline $\begin{array}{l}\text { SAVR should be considered in asymptomatic patients with normal ejection fraction and none of the above-mentioned exercise test abnor- } \\
\text { malities if the surgical risk is low and one of the following findings is present: } \\
\text { - Very severe aortic stenosis defined by } a V_{\max }>5.5 \mathrm{~m} / \mathrm{s} \\
\text { - Severe valve calcification and a rate of } \bigvee_{\max } \text { progression } \geq 0.3 \mathrm{~m} / \mathrm{s} / \text { year } \\
\text { - Markedly elevated BNP levels (>threefold age- and sex-corrected normal range) confirmed by repeated measurements } \\
\text { without other explanations } \\
\text { - Severe pulmonary hypertension (systolic pulmonary artery pressure at rest }>60 \mathrm{mmHg} \text { confirmed by invasive measure- } \\
\text { ment) without other explanation. }\end{array}$ & Ila & C \\
\hline \multicolumn{3}{|l|}{ D) Concomitant aortic valve surgery at the time of other cardiac/ascending aorta surgery } \\
\hline SAVR is indicated in patients with severe aortic stenosis undergoing CABG or surgery of the ascending aorta or of another valve. & I & C \\
\hline
\end{tabular}


BNP = B-type natriuretic peptide; CABG, coronary artery bypass grafting; CT = computed tomography; EuroSCORE = European System for Cardiac Operative Risk Evaluation; LV = left ventricular; LVEF = left ventricular ejection fraction; SAVR = surgical aortic valve replacement; STS = Society of Thoracic Surgeons; TAVI = transcatheter aortic valve implantation; $V_{\max }=$ peak transvalvular velocity.

${ }^{a}$ Class of recommendation.

'Level of evidence.

In patients with a small valve area but low gradient despite preserved LVEF, explanations for this finding other than the presence of severe aortic stenosis are frequent and must be carefully excluded. See Figure 2 and Table 6.

dSTS score (calculator: http://riskcalc.sts.org/stswebriskcalc/\#/calculate); EuroSCORE II (calculator: http://www.euroscore.org/calc.html); logistic EuroSCORE I (calculator: http://www.euroscore.org/calcge.html); scores have major limitations for practical use in this setting by insufficiently considering disease severity and not including major risk factors such as frailty, porcelain aorta, chest radiation, etc. ${ }^{103}$ EuroSCORE I markedly overestimates 30-day mortality and should therefore be replaced by the better-performing EuroSCORE II with this regard; it is nevertheless provided here for comparison, as it has been used in many TAVI studies/registries and may still be useful to identify the subgroups of patients for decision between intervention modalities and to predict 1-year mortality.

eModerate aortic stenosis is defined as a valve area of $1.0-1.5 \mathrm{~cm}^{2}$ or a mean aortic gradient of $25-40 \mathrm{mmHg}$ in the presence of normal flow conditions. However, clinical judgement is required.

\subsubsection{Indications for intervention in symptomatic aortic stenosis}

Early therapy should be strongly recommended in all symptomatic patients with severe aortic stenosis because of their dismal spontaneous prognosis. The only exceptions are patients with severe comorbidities indicating a survival of $<1$ year and patients in whom severe comorbidities or their general condition at an advanced age make it unlikely that the intervention will improve quality of life or survival.

As long as the mean gradient remains $>40 \mathrm{mmHg}$, there is virtually no lower ejection fraction limit for intervention, whether surgery or TAVI. The management of patients with low-gradient aortic stenosis is more challenging:

- In patients with low-flow, low-gradient aortic stenosis and reduced ejection fraction in whom the depressed ejection fraction is predominantly caused by excessive afterload, LV function usually improves after intervention. ${ }^{10,104}$ Conversely, improvement in LV function after intervention is uncertain if the primary cause is scarring due to extensive myocardial infarction or cardiomyopathy. Intervention is definitely advised when severe aortic stenosis is confirmed at increasing flow (true severe aortic stenosis), ${ }^{10}$ while patients who are classified as having pseudosevere aortic stenosis at increasing flow should receive conventional treatment for heart failure. ${ }^{105}$ Although the outcome of patients without flow reserve is compromised by a higher operative mortality, SAVR (as well as TAVI) has also been shown to improve ejection fraction and clinical status in such patients. ${ }^{10,78,104}$ Decision making should take into account the clinical condition (in particular the comorbidities), the degree of valve calcification, the extent of coronary disease and the feasibility of concomitant or staged revascularization. The ability to identify patients with severe aortic stenosis in this subgroup by CT calcium scoring and the availability of TAVI have lowered the threshold to intervene.

- Patients with low-flow, low-gradient aortic stenosis and preserved ejection fraction are the most challenging subgroup. Data on their natural history and outcome after surgical or catheter intervention remain controversial. ${ }^{80,83,84}$ In such cases, intervention should only be performed when symptoms are present and if comprehensive evaluation suggests significant valve obstruction (see Figure 2 and Table 6).

- Patients with normal-flow, low-gradient aortic stenosis and preserved ejection fraction data should be re-evaluated. If normal flow and low gradient are confirmed, these patients will, in general, not have severe aortic stenosis and do not benefit from intervention. $^{82,83}$

\subsubsection{Choice of intervention mode in symptomatic aortic stenosis}

The choice of the intervention mode should take into account the cardiac and extracardiac characteristics of the patient, the individual risk of surgery, which is assessed by the judgement of the Heart Team in addition to scores, the feasibility of TAVI and the local experience and outcome data.

Data on TAVI are still very limited for patients $<75$ years of age and for surgical low-risk patients, in whom SAVR remains the reference method. It has to be emphasized that younger patients differ with regard to anatomy (more bicuspid valves), which affects the results of TAVI (bicuspid valves were also in general excluded in clinical trials), and that long-term durability data for TAVI prosthetic valves are still lacking.

Available data from randomized controlled trials and large registries in elderly patients at increased surgical risk show that TAVI is superior in terms of mortality to medical therapy in extreme-risk patients, ${ }^{91}$ non-inferior or superior to surgery in high-risk patients ${ }^{94-97}$ and noninferior to surgery and even superior when transfemoral access is possible in intermediate-risk patients. ${ }^{98-102}$ In the two large studies on intermediate risk, the mean ages of patients were 82 and 80 years, ${ }^{99,102}$ mean STS scores were $5.8 \%$ and $4.5 \%{ }^{99,102}$ and a high percentage were considered frail. Thus the results are valid only for comparable patient groups. Overall, rates of vascular complications, pacemaker implantation and paravalvular regurgitation were significantly higher for TAVI, while the degree of excess depended on the device used. ${ }^{101,102}$ On the other hand, severe bleeding, acute kidney injury and new-onset atrial fibrillation were significantly more frequent with surgery, whereas no difference was observed in the rate of cerebrovascular events. ${ }^{101,102}$ The favourable results of TAVI have been reproduced in multiple large-scale, nationwide registries supporting the generalizability of outcomes observed in randomized controlled trials. This favours the use of TAVI over surgery in elderly patients at increased surgical risk. However, the final decision between SAVR and TAVI (including the choice of access route) should be made by the Heart Team after careful individual evaluation. Table 7 provides aspects that should be considered for the individual decision. Balloon valvuloplasty may be considered as a bridge to surgery or TAVI, or diagnostically.

\subsubsection{Asymptomatic aortic stenosis}

Management of asymptomatic severe aortic stenosis remains controversial. The available studies do not provide convincing data to 
Table 7 Aspects to be considered by the Heart Team for the decision between SAVR and TAVI in patients at increased surgical risk (see Table of Recommendations in section 5.2.)

\begin{tabular}{|c|c|c|}
\hline & $\begin{array}{l}\text { Favours } \\
\text { TAVI }\end{array}$ & $\begin{array}{l}\text { Favours } \\
\text { SAVR }\end{array}$ \\
\hline \multicolumn{3}{|l|}{ Clinical characteristics } \\
\hline $\begin{array}{l}\text { STS/EuroSCORE II }<4 \% \\
{\text { (logistic EuroSCORE I }<10 \%)^{\text {a }}}\end{array}$ & & + \\
\hline $\begin{array}{l}\text { STS/EuroSCORE II } \geq 4 \% \\
{\text { (logistic EuroSCORE I } \geq 10 \%)^{\mathrm{a}}}\end{array}$ & + & \\
\hline $\begin{array}{l}\text { Presence of severe comorbidity } \\
\text { (not adequately reflected by scores) }\end{array}$ & + & \\
\hline Age $<75$ years & & + \\
\hline Age $\geq 75$ years & + & \\
\hline Previous cardiac surgery & + & \\
\hline Frailty $^{b}$ & + & \\
\hline $\begin{array}{l}\text { Restricted mobility and conditions that may } \\
\text { affect the rehabilitation process after the } \\
\text { procedure }\end{array}$ & + & \\
\hline Suspicion of endocarditis & & + \\
\hline \multicolumn{3}{|l|}{ Anatomical and technical aspects } \\
\hline Favourable access for transfemoral TAVI & + & \\
\hline Unfavourable access (any) for TAVI & & + \\
\hline Sequelae of chest radiation & + & \\
\hline Porcelain aorta & + & \\
\hline $\begin{array}{l}\text { Presence of intact coronary bypass grafts at } \\
\text { risk when sternotomy is performed }\end{array}$ & + & \\
\hline Expected patient-prosthesis mismatch & + & \\
\hline Severe chest deformation or scoliosis & + & \\
\hline $\begin{array}{l}\text { Short distance between coronary ostia and } \\
\text { aortic valve annulus }\end{array}$ & & + \\
\hline $\begin{array}{l}\text { Size of aortic valve annulus out of range for } \\
\text { TAVI }\end{array}$ & & + \\
\hline Aortic root morphology unfavourable for TAVI & & + \\
\hline $\begin{array}{l}\text { Valve morphology (bicuspid, degree } \\
\text { of calcification, calcification pattern) } \\
\text { unfavourable for TAVI }\end{array}$ & & + \\
\hline Presence of thrombi in aorta or LV & & + \\
\hline \multicolumn{3}{|c|}{$\begin{array}{l}\text { Cardiac conditions in addition to aortic stenosis that } \\
\text { require consideration for concomitant intervention }\end{array}$} \\
\hline $\begin{array}{l}\text { Severe } C A D \text { requiring revascularization by } \\
C A B G\end{array}$ & & + \\
\hline $\begin{array}{l}\text { Severe primary mitral valve disease, which } \\
\text { could be treated surgically }\end{array}$ & & + \\
\hline Severe tricuspid valve disease & & + \\
\hline Aneurysm of the ascending aorta & & + \\
\hline Septal hypertrophy requiring myectomy & & + \\
\hline
\end{tabular}

$C A B G=$ coronary artery bypass grafting; $C A D=$ coronary artery disease; EuroSCORE = European System for Cardiac Operative Risk Evaluation; $L V=$ left ventricle; SAVR = surgical aortic valve replacement; STS = Society of Thoracic Surgeons; $\mathrm{TAVI}$ = transcatheter aortic valve implantation.

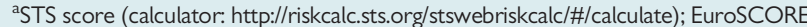
II (calculator: http://www.euroscore.org/calc.html); logistic EuroSCORE I (calculator: http://www.euroscore.org/calcge.html); scores have major limitations for practical use in this setting by insufficiently considering disease severity and not including major risk factors such as frailty, porcelain aorta, chest radiation etc. ${ }^{103}$ EuroSCORE I markedly overestimates 30-day mortality and should therefore be replaced by the better performing EuroSCORE II with this regard; it is nevertheless provided here for comparison as it has been used in many TAVI studies/registries and may still be useful to identify the subgroups of patients for decision between intervention modalities and to predict 1-year mortality.

bee section 3.3, general comments, for frailty assessment. support the general recommendation of early SAVR, even in patients with asymptomatic very severe aortic stenosis. ${ }^{92,106}$ The decision to operate on asymptomatic patients requires careful weighing of the benefits against the risks. This section refers only to patients who are candidates for SAVR, as TAVI is not recommended in asymptomatic patients. Early elective surgery is indicated in asymptomatic patients with depressed LV function not due to other causes and in patients who develop symptoms during exercise testing. ${ }^{85,107}$

Predictors of symptom development and adverse outcomes in asymptomatic patients include clinical characteristics (older age, presence of atherosclerotic risk factors), echocardiographic parameters (valve calcification, peak aortic jet velocity, ${ }^{92,108}$ LVEF, rate of haemodynamic progression, ${ }^{92}$ increase in mean gradient $>20 \mathrm{mmHg}$ with exercise, ${ }^{86}$ excessive LV hypertrophy, ${ }^{109}$ abnormal longitudinal LV function ${ }^{110}$ and pulmonary hypertension ${ }^{111}$ ) and biomarkers (elevated plasma levels of natriuretic peptides, although the precise cutoff values have not yet been well defined ${ }^{89,90}$ ). When early elective surgery is considered in patients with normal exercise performance because of the presence of such outcome predictors, the operative risk should be low (see table of recommendations in section 5.2 Indications for interventions in aortic stenosis). In patients without predictive factors, watchful waiting appears safe and early surgery is unlikely to be beneficial.

\subsection{Medical therapy}

No medical therapy for aortic stenosis can improve outcome compared with the natural history. Randomized trials have consistently shown that statins do not affect the progression of aortic stenosis. ${ }^{112}$ Patients with symptoms of heart failure who are unsuitable candidates for surgery or TAVI or who are currently awaiting surgical or catheter intervention should be medically treated according to the heart failure guidelines. ${ }^{113}$ Coexisting hypertension should be treated. Medical treatment should be carefully titrated to avoid hypotension and patients should be re-evaluated frequently. Maintenance of sinus rhythm is important.

\subsection{Serial testing}

In the asymptomatic patient, the wide variability in the rate of progression of aortic stenosis stresses the need for patients to be carefully educated about the importance of follow-up and reporting symptoms as soon as they develop. Stress tests should determine the recommended level of physical activity. Follow-up evaluation should focus on haemodynamic progression, LV function and hypertrophy and dimensions of the ascending aorta.

Asymptomatic severe aortic stenosis should be re-evaluated at least every 6 months for the occurrence of symptoms (change in exercise tolerance, ideally using exercise testing if symptoms are doubtful) and change in echocardiographic parameters. Measurement of natriuretic peptides should be considered.

In the presence of significant calcification, mild and moderate aortic stenosis should be re-evaluated yearly. In younger patients with mild aortic stenosis and no significant calcification, intervals may be extended to $2-3$ years. 


\subsection{Special patient populations}

Combined SAVR and CABG carry a higher risk than isolated SAVR. However, SAVR late after CABG is also associated with significantly increased risk. Data from retrospective analyses indicate that patients in whom CABG is indicated and who have moderate aortic stenosis will in general benefit from concomitant SAVR. It has also been suggested that if age is $<70$ years and, more importantly, an average rate of aortic stenosis progression of $5 \mathrm{mmHg} /$ year is documented, patients may benefit from valve replacement at the time of coronary surgery once the baseline peak gradient exceeds $30 \mathrm{mmHg}$. ${ }^{114}$ Individual judgement is recommended, taking into consideration BSA, haemodynamic data, leaflet calcification, aortic stenosis progression rate, patient life expectancy and associated comorbidities, as well as the individual risk of either concomitant valve replacement or late reoperation. ${ }^{93}$ Patients with severe symptomatic aortic stenosis and diffuse CAD that cannot be revascularized should not be denied SAVR or TAVI.

Combined $\mathrm{PCl}$ and TAVI has been shown to be feasible but requires more data before a firm recommendation can be made. The chronology of interventions should be the subject of individualized discussion based on the patient's clinical condition, extent of CAD and myocardium at risk.

When mitral regurgitation is associated with severe aortic stenosis, its severity may be overestimated in the presence of the high ventricular pressures and careful quantification is required. As long as there are no morphological leaflet abnormalities (flail or prolapse, postrheumatic changes or signs of infective endocarditis), mitral annulus dilatation or marked abnormalities of LV geometry, surgical intervention on the mitral valve is in general not necessary. Non-severe secondary mitral regurgitation mostly improves after the aortic valve is treated. In patients with severe mitral regurgitation, combined or sequential TAVI and percutaneous mitral edge-to-edge repair have been demonstrated to be feasible, but there is not enough experience to make recommendations.

Concomitant aneurysm/dilatation of the ascending aorta requires the same treatment as in aortic regurgitation (see section 4).

For congenital aortic stenosis, see the ESC guidelines on grown-up congenital heart disease. ${ }^{115}$

\section{Key points}

- The diagnosis of severe aortic stenosis requires consideration of AVA together with flow rate, pressure gradients (the most robust measurement), ventricular function, size and wall thickness, degree of valve calcification and blood pressure, as well as functional status.

- The assessment of the severity of aortic stenosis in patients with low gradient and preserved ejection fraction remains particularly challenging.

- The strongest indication for intervention remains symptoms of aortic stenosis (spontaneous or on exercise testing).

- The presence of predictors of rapid symptom development can justify early surgery in asymptomatic patients, particularly when surgical risk is low.

- Although current data favour TAVI in elderly patients who are at increased risk for surgery, particularly when a transfemoral access is possible, the decision between TAVI and SAVR should be made by the Heart Team after careful, comprehensive evaluation of the patient, weighing individually the risks and benefits.

\section{Gaps in evidence}

- The impact of earlier markers of LV dysfunction on postoperative outcome requires further research.

- The identification of patients with low-gradient aortic stenosis who have severe stenosis and would benefit from intervention requires improvement.

- The criteria for identification of patients who would benefit from early elective surgery in asymptomatic severe aortic stenosis requires further research.

- Long-term follow-up after TAVI is required; in particular, the long-term durability of the valves needs to be studied.

- Criteria for the decision between TAVI and SAVR in patients at increased operative risk who are eligible for both must be refined and must be studied in surgical low-risk patients.

- Criteria for when TAVI should no longer be performed since it would be futile need to be further defined.

\section{Mitral regurgitation}

Mitral regurgitation is the second-most frequent indication for valve surgery in Europe. ${ }^{47}$ It is essential to distinguish primary from secondary mitral regurgitation, particularly regarding surgical and transcatheter interventional management. ${ }^{116}$

\subsection{Primary mitral regurgitation}

In primary mitral regurgitation, one or several components of the mitral valve apparatus are directly affected. The most frequent aetiology is degenerative (prolapse, flail leaflet). Endocarditis as one of the causes of primary mitral regurgitation is discussed in specific ESC guidelines. $^{28}$

\subsubsection{Evaluation}

Echocardiography is the principal investigation used to assess the severity and mechanism of mitral regurgitation, its consequences for the LV (function and remodelling), left atrium (LA) and pulmonary circulation, as well as the likelihood of repair.

Quantification should be performed in an integrative way, including qualitative, semi-quantitative and quantitative parameters. The criteria for defining severe primary mitral regurgitation are summarized in Table 4. ${ }^{2,7}$

A precise anatomical description of the lesions, using the segmental and functional anatomy according to the Carpentier classification, ${ }^{2,7}$ should be performed to assess the feasibility of repair. TTE also assesses mitral annular dimensions and the presence of calcification.

TTE is diagnostic in most cases, but TOE is recommended, particularly in the presence of suboptimal image quality. ${ }^{117}$ Threedimensional echocardiography provides additional information for selecting the appropriate repair strategy.

The consequences of mitral regurgitation on ventricular function are assessed by measuring LV size and ejection fraction. LA volume, systolic pulmonary artery pressure, tricuspid regurgitation and annular size and RV function are important additional parameters.

Determination of functional capacity and symptoms assessed by cardiopulmonary exercise testing may be useful in asymptomatic patients. Exercise echocardiography is useful to quantify exerciseinduced changes in mitral regurgitation, ${ }^{118}$ in systolic pulmonary 
artery pressure and in LV function. It may be particularly helpful in patients with symptoms and uncertainty about the severity of mitral regurgitation based on measurements at rest. In asymptomatic patients, the significant increase of pulmonary artery pressure with exercise $(>60 \mathrm{mmHg})$ has been reported to be of prognostic value. ${ }^{119}$ The use of global longitudinal strain could be of potential interest for the detection of subclinical LV dysfunction but is limited by inconsistent algorithms used by different echocardiographic systems.

Neurohormonal activation is observed in mitral regurgitation, with a potential value of elevated BNP levels and a change in BNP as predictors of outcome (particularly of symptom onset). In particular, low plasma BNP has a high negative predictive value and may be helpful in the follow-up of asymptomatic patients. ${ }^{120}$

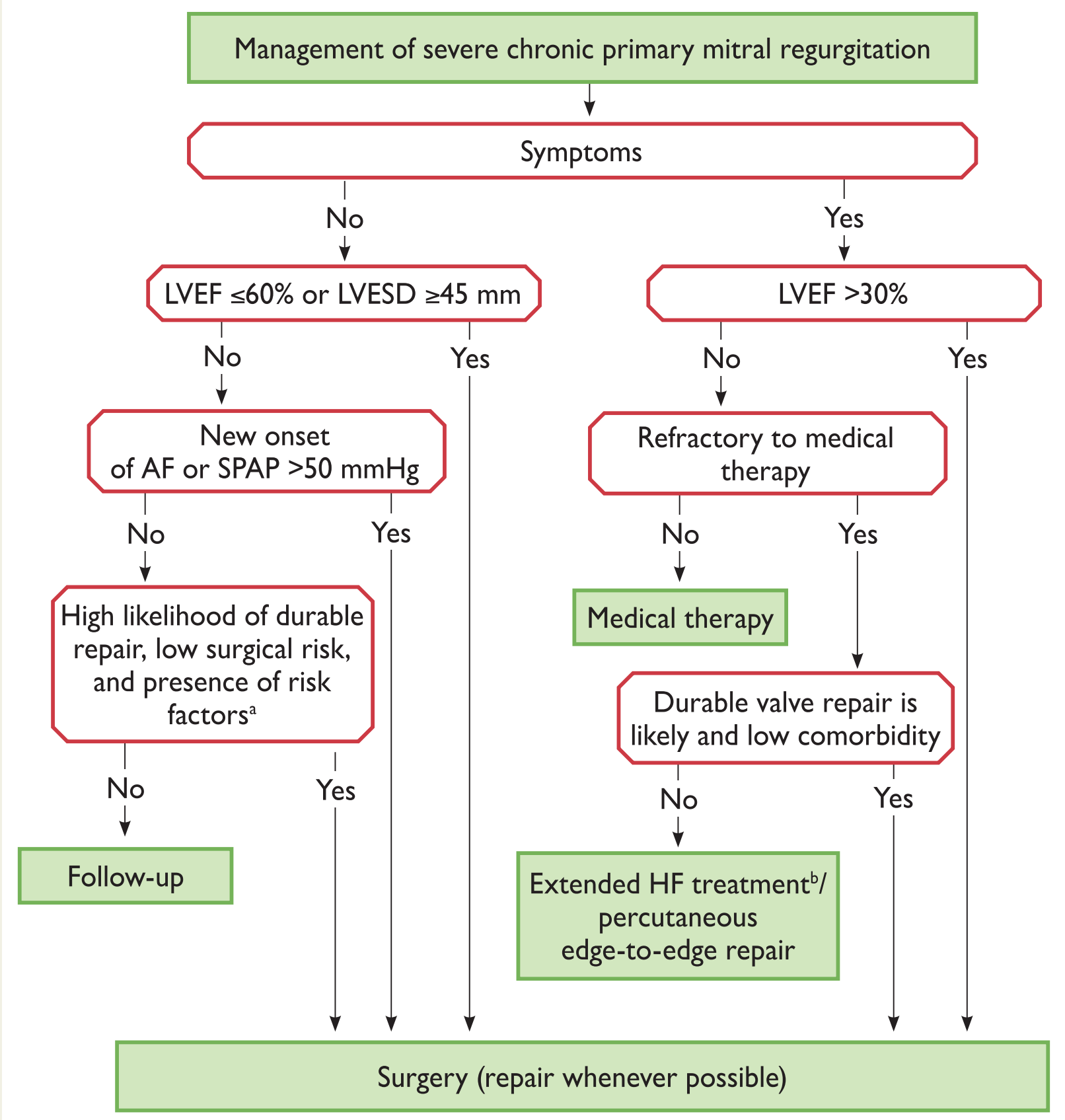

Figure 4 Management of severe chronic primary mitral regurgitation. AF = atrial fibrillation; BSA = body surface area; $C R T=$ cardiac resynchronization therapy; HF = heart failure; LA = left atrial; LVEF = left ventricular ejection fraction; LVESD = left ventricular end-systolic diameter; SPAP = systolic pulmonary arterial pressure.

a When there is a high likelihood of durable valve repair at a low-risk, valve repair should be considered (Ila C) in patients with LVESD $\geq 40 \mathrm{~mm}$ and one of the following is present: flail leaflet or LA volume $\geq 60 \mathrm{~mL} / \mathrm{m}^{2}$ BSA at sinus rhythm.

${ }^{\mathrm{b}}$ Extended HF management includes the following: CRT; ventricular assist devices; cardiac restraint devices; heart transplantation. 
As echocardiographic measures of pulmonary pressure may show disagreement with invasive measures, the measurement should be invasively confirmed by right-heart catheterization if this is the only indication for surgery.

\subsubsection{Indications for intervention}

Urgent surgery is indicated in patients with acute severe mitral regurgitation. In the case of papillary muscle rupture as the underlying disease, valve replacement is in general required.

Indications for surgery in severe chronic primary mitral regurgitation are shown in the following table of recommendations (indications for intervention in severe primary mitral regurgitation) and in Figure 4. Surgery is obviously indicated in symptomatic patients with severe primary mitral regurgitation. ${ }^{121}$ An LVEF $\leq 60 \%$ or LVESD $\geq 45 \mathrm{~mm}^{122}$ atrial fibrillation ${ }^{123}$ and a systolic pulmonary pressure $\geq 50 \mathrm{mmHg}^{124}$ predict a worse postoperative outcome independent of the symptomatic status and have therefore become triggers for surgery in asymptomatic patients. In patients with flail leaflet, an LVESD of 40-44 mm has been reported to predict a worse outcome compared with LVESD $<40 \mathrm{~mm}{ }^{125}$ Significant LA dilatation despite sinus rhythm has also been found to be a predictor of outcome. ${ }^{124}$ In the presence of these two latter triggers, surgery should only be considered in heart valve centres and if surgical risk is low. An increase in systolic pulmonary artery pressure $>60 \mathrm{mmHg}$ on exercise echocardiography has also been proposed for risk stratification. ${ }^{119}$ However, criteria that may indicate surgery have not been sufficiently well defined to be included in the current recommendations.

Watchful waiting is a safe strategy in asymptomatic patients with severe primary mitral regurgitation and none of the above indications for surgery, ${ }^{126}$ and ideally patients are followed in the setting of a heart valve centre. ${ }^{32}$

Despite the absence of a randomized comparison between the results of valve replacement and repair, it is widely accepted that, when feasible, valve repair is the preferred treatment. Achieving a durable valve repair is essential. Degenerative mitral regurgitation due to segmental valve prolapse can be repaired with a low risk of mitral regurgitation recurrence and reoperation. The reparability of rheumatic lesions, extensive valve prolapse and-even more somitral regurgitation with leaflet calcification or extensive annular calcification is more challenging. Patients with a predictably complex repair should undergo surgery in experienced repair centres with high repair rates, low operative mortality and a record of durable results. ${ }^{127,128}$ When repair is not feasible, mitral valve replacement with preservation of the subvalvular apparatus is favoured. Additional tricuspid valve repair should be performed as indicated in section 8.2 (see table of recommendations on indications for tricuspid valve surgery).

Transcatheter mitral valve interventions have been developed to correct primary mitral regurgitation either through a transseptal or a transapical approach. Among the transcatheter procedures, currently only the edge-to-edge mitral repair is widely adopted. ${ }^{129}$ Experience with transcatheter annuloplasty, transapical chordal implantation or valve replacement is still limited and general recommendations cannot yet be made. Transcatheter mitral valve treatment should be discussed by the Heart Team in symptomatic patients who are at high surgical risk or are inoperable. Percutaneous edge-to-edge repair is generally safe and can improve symptoms and provide reverse LV remodelling. However, the rate of residual mitral regurgitation up to 5 years is higher than with surgical repair. ${ }^{130}$

\section{Indications for intervention in severe primary mitral regurgitation}

\begin{tabular}{|c|c|c|}
\hline Recommendations & Class $^{a}$ & Level $^{b}$ \\
\hline $\begin{array}{l}\text { Mitral valve repair should be the preferred } \\
\text { technique when the results are expected to be } \\
\text { durable. }\end{array}$ & I & C \\
\hline $\begin{array}{l}\text { Surgery is indicated in symptomatic patients } \\
\text { with LVEF }>30 \% .{ }^{121,131,132}\end{array}$ & I & B \\
\hline $\begin{array}{l}\text { Surgery is indicated in asymptomatic patients } \\
\text { with LV dysfunction (LVESD } \geq 45 \mathrm{~mm}^{\mathrm{c}} \text { and/or } \\
\text { LVEF } \leq 60 \%){ }^{122,131}\end{array}$ & I & B \\
\hline 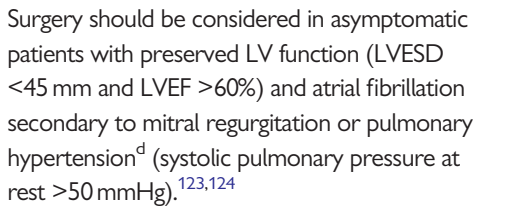 & Ila & B \\
\hline $\begin{array}{l}\text { Surgery should be considered in asymptomatic } \\
\text { patients with preserved LVEF ( }>60 \% \text { ) and LVESD } \\
40-44 \mathrm{~mm}^{\mathrm{c}} \text { when a durable repair is likely, surgi- } \\
\text { cal risk is low, the repair is performed in a heart } \\
\text { valve centre and at least one of the following find- } \\
\text { ings is present: } \\
\text { - flail leaflet or } \\
\text { - presence of significant LA dilatation (vol- } \\
\text { ume index } \geq 60 \mathrm{~mL} / \mathrm{m}^{2} \mathrm{BSA} \text { ) in sinus } \\
\text { rhythm. }\end{array}$ & Ila & C \\
\hline $\begin{array}{l}\text { Mitral valve repair should be considered in } \\
\text { symptomatic patients with severe LV dysfunc- } \\
\text { tion (LVEF }<30 \% \text { and/or LVESD }>55 \mathrm{~mm} \text { ) } \\
\text { refractory to medical therapy when the likeli- } \\
\text { hood of successful repair is high and comorbid- } \\
\text { ity low. }\end{array}$ & Ila & C \\
\hline $\begin{array}{l}\text { Mitral valve replacement may be considered in } \\
\text { symptomatic patients with severe LV dysfunc- } \\
\text { tion (LVEF }<30 \% \text { and/or LVESD }>55 \mathrm{~mm} \text { ) } \\
\text { refractory to medical therapy when the likeli- } \\
\text { hood of successful repair is low and comorbid- } \\
\text { ity low. }\end{array}$ & Ilb & C \\
\hline $\begin{array}{l}\text { Percutaneous edge-to-edge procedure may be } \\
\text { considered in patients with symptomatic } \\
\text { severe primary mitral regurgitation who fulfil } \\
\text { the echocardiographic criteria of eligibility and } \\
\text { are judged inoperable or at high surgical risk by } \\
\text { the Heart Team, avoiding futility. }\end{array}$ & Ilb & C \\
\hline
\end{tabular}

$B S A=$ body surface area; $L A=$ left atrial; $L V=$ left ventricular; $L V E F=$ left ventricular ejection fraction; LVESD = left ventricular end-systolic diameter; SPAP = systolic pulmonary artery pressure.

${ }^{a}$ Class of recommendation.

bLevel of evidence.

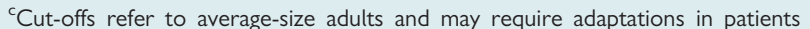
with unusually small or large stature.

dIf an elevated SPAP is the only indication for surgery, the value should be confirmed by invasive measurement. 


\subsubsection{Medical therapy}

In acute mitral regurgitation, nitrates and diuretics are used to reduce filling pressures. Sodium nitroprusside reduces afterload and regurgitant fraction. Inotropic agents and an intra-aortic balloon pump are of use in hypotension and haemodynamic instability.

In chronic mitral regurgitation with good ventricular function, there is no evidence to support the prophylactic use of vasodilators, including ACE inhibitors. However, ACE inhibitors should be considered when heart failure has developed in patients who are not suitable for surgery or when symptoms persist after surgery. Betablockers and spironolactone (or eplerenone) should also be considered as appropriate.

\subsubsection{Serial testing}

Asymptomatic patients with severe mitral regurgitation and LVEF $>60 \%$ should be followed clinically and echocardiographically every 6 months, ideally in the setting of a heart valve centre. Closer followup is indicated if no previous evaluation is available and when measured variables show significant dynamic changes or are close to the thresholds. When guideline indications for surgery are reached, early surgery - within 2 months - is associated with better outcomes. ${ }^{133}$ Asymptomatic patients with moderate mitral regurgitation and preserved LV function can be followed on a yearly basis and echocardiography should be performed every $1-2$ years.

\subsection{Secondary mitral regurgitation}

In secondary mitral regurgitation (previously also referred to as 'functional mitral regurgitation'), the valve leaflets and chordae are structurally normal and mitral regurgitation results from an imbalance between closing and tethering forces on the valve secondary to alterations in LV geometry. ${ }^{134}$ It is most commonly seen in dilated or ischaemic cardiomyopathies. Annular dilatation in patients with chronic atrial fibrillation and LA enlargement can also be an underlying mechanism.

\subsubsection{Evaluation}

Echocardiography is essential to establish the diagnosis of secondary mitral regurgitation. In secondary mitral regurgitation, lower thresholds have been proposed to define severe mitral regurgitation compared with primary mitral regurgitation $\left[20 \mathrm{~mm}^{2}\right.$ for effective regurgitant orifice area (EROA) and $30 \mathrm{~mL}$ for regurgitant volume], owing to their association with prognosis. ${ }^{135}$ However, it is unclear if prognosis is independently affected by mitral regurgitation compared with LV dysfunction. So far, no survival benefit has been confirmed for reduction of secondary mitral regurgitation.

For isolated mitral valve treatment (surgery or percutaneous edge-to-edge repair) in secondary mitral regurgitation, thresholds of severity of mitral regurgitation for intervention still need to be validated in clinical trials. The severity of secondary mitral regurgitation should be reassessed after optimized medical treatment. The severity of tricuspid regurgitation and RV size and function should also be evaluated.

Secondary mitral regurgitation is a dynamic condition; echocardiographic quantification of mitral regurgitation during exercise may provide prognostic information of dynamic characteristics. Myocardial viability testing may be useful in patients with ischaemic secondary mitral regurgitation who are candidates for revascularization.

\subsubsection{Indications for intervention}

The presence of chronic secondary mitral regurgitation is associated with impaired prognosis. ${ }^{135}$ However, in contrast to primary mitral regurgitation, there is currently no evidence that a reduction of secondary mitral regurgitation improves survival. The limited data regarding secondary mitral regurgitation result in a lower level of evidence for treatment recommendations (see table of recommendations on indications for mitral valve intervention in chronic secondary mitral regurgitation) and highlight the importance of decision making by the Heart Team. Heart failure and electrophysiology specialists should be involved.

In patients with CAD undergoing revascularization, the evaluation and decision to treat (or not to treat) ischaemic mitral regurgitation should be made before surgery, as general anaesthesia may significantly reduce the severity of regurgitation. When mitral regurgitation severity is assessed intraoperatively, the use of acute volume challenge and an increase in afterload may be helpful.

The optimal surgical approach remains controversial. ${ }^{136}$ While mitral valve repair with an undersized complete ring to restore leaflet coaptation and valve competence is the preferred technique, valve replacement should be considered in patients with echocardiographic risk factors for residual or recurrent mitral regurgitation. ${ }^{2}$

Indications for surgery in secondary mitral regurgitation are particularly restrictive when concomitant revascularization is not an option, owing to significant operative mortality, high rates of recurrent mitral regurgitation and the absence of a proven survival benefit. $^{137,138}$

Percutaneous edge-to-edge repair for secondary mitral regurgitation is a low-risk option, but its efficacy to reduce mitral regurgitation remains inferior to surgery. ${ }^{139}$ It can improve symptoms, functional capacity and quality of life and may induce reverse LV remodelling. ${ }^{140}$ Similar to surgery, a survival benefit compared with 'optimal' medical therapy according to current guidelines ${ }^{113}$ has not yet been proven.

In patients with markedly reduced LV function (ejection fraction $\leq 30 \%$ ) and no option for revascularization who remain symptomatic despite optimal heart-failure treatment [including cardiac resynchronization therapy (CRT) when indicated], the decision between palliative mitral regurgitation treatment-catheter-based or surgical, ventricular assist devices, heart transplantation - and continued conservative therapy should be made by the Heart Team after careful individual evaluation of the patient. Valve intervention is generally not an option when the ejection fraction is $<15 \%$.

There is continuing debate regarding the management of moderate ischaemic mitral regurgitation in patients undergoing $C A B G$. A recent randomized controlled trial could not show a benefit of concomitant valve surgery. ${ }^{141}$ Surgery is more likely to be considered if myocardial viability is present and if comorbidity is low. In patients capable of exercising, exercise-induced dyspnoea and a large increase in mitral regurgitation severity and systolic pulmonary artery pressure favour combined surgery. 


\section{Indications for mitral valve intervention in chronic sec- ondary mitral regurgitation ${ }^{\mathrm{a}}$}

\begin{tabular}{|c|c|c|}
\hline Recommendations & Class $^{b}$ & Level $^{c}$ \\
\hline $\begin{array}{l}\text { Surgery is indicated in patients with severe } \\
\text { secondary mitral regurgitation undergoing } \\
\text { CABG and LVEF }>30 \% \text {. }\end{array}$ & I & C \\
\hline $\begin{array}{l}\text { Surgery should be considered in sympto- } \\
\text { matic patients with severe secondary mitral } \\
\text { regurgitation, LVEF < } 30 \% \text { but with an } \\
\text { option for revascularization and evidence of } \\
\text { myocardial viability. }\end{array}$ & IIa & C \\
\hline $\begin{array}{l}\text { When revascularization is not indicated, } \\
\text { surgery may be considered in patients with } \\
\text { severe secondary mitral regurgitation and } \\
\text { LVEF }>30 \% \text { who remain symptomatic } \\
\text { despite optimal medical management } \\
\text { (including CRT if indicated) and have a low } \\
\text { surgical risk. }\end{array}$ & Ilb & C \\
\hline $\begin{array}{l}\text { When revascularization is not indicated and } \\
\text { surgical risk is not low, a percutaneous } \\
\text { edge-to-edge procedure may be considered } \\
\text { in patients with severe secondary mitral } \\
\text { regurgitation and LVEF }>30 \% \text { who remain } \\
\text { symptomatic despite optimal medical man- } \\
\text { agement (including CRT if indicated) and } \\
\text { who have a suitable valve morphology by } \\
\text { echocardiography, avoiding futility. }\end{array}$ & Ilb & C \\
\hline $\begin{array}{l}\text { In patients with severe secondary mitral } \\
\text { regurgitation and LVEF }<30 \% \text { who remain } \\
\text { symptomatic despite optimal medical } \\
\text { management (including CRT if indicated) } \\
\text { and who have no option for revasculariza- } \\
\text { tion, the Heart Team may consider a percu- } \\
\text { taneous edge-to-edge procedure or valve } \\
\text { surgery after careful evaluation for a ventric- } \\
\text { ular assist device or heart transplant accord- } \\
\text { ing to individual patient characteristics. }\end{array}$ & Ilb & C \\
\hline
\end{tabular}

$C A B G=$ coronary artery bypass grafting; $C R T=$ cardiac resynchronization therapy; $\mathrm{LVEF}=$ left ventricular ejection fraction.

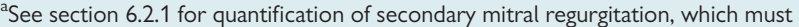
always be performed under optimal treatment.

${ }^{b}$ Class of recommendation.

'Level of evidence.

\subsubsection{Medical therapy}

Optimal medical therapy in line with the guidelines for the management of heart failure ${ }^{113}$ should be the first step in the management of all patients with secondary mitral regurgitation. Indications for CRT should be evaluated in accordance with related guidelines. ${ }^{113}$ If symptoms persist after optimization of conventional heart failure therapy, options for mitral valve intervention should be evaluated.

\section{Key points}

- Echocardiography is essential to assess the aetiology of mitral regurgitation, as well as valve anatomy and function. An integrative approach is needed to assess the severity of mitral regurgitation.

- Indication for intervention in primary mitral regurgitation is guided by symptoms and risk stratification that includes the assessment of ventricular function and size, atrial fibrillation, systolic pulmonary pressure and LA size.

- In secondary mitral regurgitation, there is no conclusive evidence for a survival benefit after mitral valve intervention. Mitral surgery is recommended concomitantly in patients with an indication for CABG and may be considered in patients who are symptomatic despite optimal medical therapy (including CRT if indicated) or who have a low surgical risk when revascularization is not indicated.

- Mitral valve repair is the preferred method, but mitral valve replacement should be considered in patients with unfavourable morphological characteristics.

- Outcomes of mitral valve repair depend on surgeon experience and centre-related volume.

- Percutaneous edge-to-edge repair may be considered in patients at high surgical risk, avoiding futility.

\section{Gaps in evidence}

- The potential role of elective mitral valve surgery in asymptomatic patients with severe primary mitral regurgitation with preserved ventricular size and function who are in sinus rhythm and have not developed a high pulmonary artery pressure requires investigation in a randomized controlled trial.

- The impact of earlier markers of LV dysfunction on postoperative outcome requires further research.

- The thresholds to define severe secondary mitral regurgitation are controversial and need to be evaluated with regards to their impact on prognosis after mitral valve intervention.

- The potential impact of mitral valve intervention (surgery and catheter intervention) on survival in patients with secondary mitral regurgitation needs to be evaluated.

- The new percutaneous valve repair and valve implantation techniques require further evaluation.

\section{Mitral stenosis}

The incidence of rheumatic mitral stenosis has greatly decreased in industrialized countries. ${ }^{142}$ Degenerative calcific mitral valve disease is now encountered mainly in elderly patients. ${ }^{143}$ Percutaneous mitral commissurotomy (PMC) has had a significant impact on the management of rheumatic mitral stenosis.

\subsection{Evaluation}

Echocardiography is the preferred method for diagnosing mitral stenosis and for assessing its severity and haemodynamic consequences. However, several specific issues should be considered. Valve area using planimetry is the reference measurement of mitral stenosis severity, whereas mean transvalvular gradient and pulmonary pressures reflect its consequences and have a prognostic value. ${ }^{3}$ TTE usually provides sufficient information for routine management. 


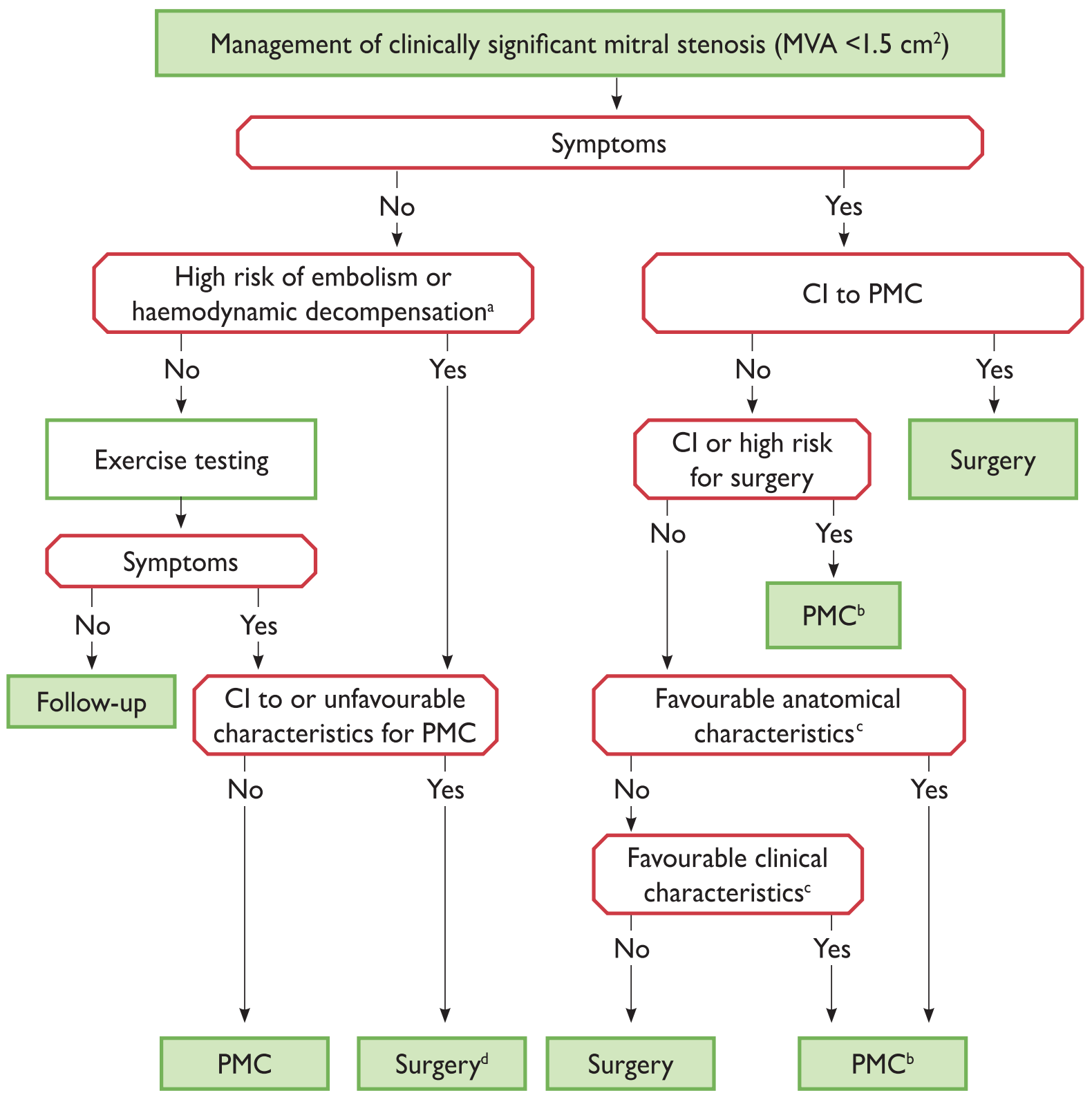

Figure 5 Management of clinically significant mitral stenosis. $\mathrm{Cl}=$ contra-indication; $\mathrm{MS}=$ mitral stenosis; $\mathrm{PMC}=$ percutaneous mitral commissurotomy.

${ }^{a}$ High thromboembolic risk: history of systemic embolism, dense spontaneous contrast in the left atrium, new-onset atrial fibrillation. High-risk of haemodynamic decompensation: systolic pulmonary pressure $>50 \mathrm{mmHg}$ at rest, need for major non-cardiac surgery, desire for pregnancy. 'burgical commissurotomy may be considered by experienced surgical teams or in patients with contra-indications to PMC. ${ }^{\mathrm{C}} \mathrm{See}$ table of recommendations on indications for PMC and mitral valve surgery in clinically significant mitral stenosis in section 7.2. dSurgery if symptoms occur for a low level of exercise and operative risk is low. 
Scoring systems have been developed to help assess suitability for PMC. ${ }^{144-146}$ TOE should be performed to exclude LA thrombus before PMC or after an embolic episode. Echocardiography also plays an important role in monitoring the results of PMC during the procedure. Stress testing is indicated in patients with no symptoms or symptoms equivocal or discordant with the severity of mitral stenosis. Exercise echocardiography may provide additional objective information by assessing changes in mitral gradient and pulmonary artery pressure.

\subsection{Indications for intervention}

The type of treatment, as well as its timing, should be decided on the basis of clinical characteristics, valve anatomy and local expertise. In general, indication for intervention should be limited to patients with clinically significant (moderate to severe) mitral stenosis (valve area $<1.5 \mathrm{~cm}^{2}$ ). However, PMC may be considered in symptomatic patients with a valve area $>1.5 \mathrm{~cm}^{2}$ if symptoms cannot be explained by another cause and if the anatomy is favourable.

The management of clinically significant mitral stenosis is summarized in Figure 5 and the indications and contraindications for PMC are provided in the table of recommendations on indications for PMC and mitral valve surgery in clinically significant mitral stenosis and in Table 8. Intervention should be performed in symptomatic patients. Most patients with favourable valve anatomy currently undergo PMC, however, open commissurotomy may be preferred by experienced surgeons in young patients with mild to moderate mitral regurgitation.

In patients with unfavourable anatomy, decision making as to the type of intervention is still a matter of debate and must take into account the multifactorial nature of predicting the results of PMC. ${ }^{147-149}$ PMC should be considered as an initial treatment for selected patients with mild to moderate calcification or impaired subvalvular apparatus who have otherwise favourable clinical characteristics. Surgery, which is mostly valve replacement, is indicated in the other patients.

Owing to the small but definite risk inherent to PMC, truly asymptomatic patients, as assessed using stress testing, are usually not candidates for the procedure, except in cases where there is increased risk of systemic embolism or haemodynamic decompensation. In such patients, PMC should only be performed if they have favourable characteristics and if it is undertaken by experienced operators.

In asymptomatic patients with mitral stenosis, surgery is limited to those rare patients at high risk of cardiac complications who have contraindications for PMC and are at low risk for surgery.

The most important contraindication to PMC is LA thrombus (Table 8). However, when the thrombus is located in the LA appendage, PMC may be considered in patients without urgent need for intervention, provided repeat TOE shows the thrombus has disappeared after 1-3 months of oral anticoagulation. Surgery is indicated if the thrombus persists.
Indications for PMC and mitral valve surgery in clinically significant (moderate or severe) mitral stenosis (valve area $\leq 1.5 \mathrm{~cm}^{2}$ )

\begin{tabular}{|l|c|c|}
\hline Recommendations & Class $^{\mathbf{a}}$ & Level $^{\mathbf{b}}$ \\
\hline $\begin{array}{l}\text { PMC is indicated in symptomatic patients } \\
\text { without unfavourable characteristics }\end{array}$ for & I & B \\
PMC. $^{144,146,148}$ & & \\
\hline $\begin{array}{l}\text { PMC is indicated in any symptomatic } \\
\text { patients with a contraindication or a high } \\
\text { risk for surgery. }\end{array}$ & I & C \\
\hline $\begin{array}{l}\text { Mitral valve surgery is indicated in sympto- } \\
\text { matic patients who are not suitable for }\end{array}$ & I & C \\
PMC. & & \\
\hline $\begin{array}{l}\text { PMC should be considered as initial treat- } \\
\text { ment in symptomatic patients with subopti- } \\
\text { mal anatomy but no unfavourable clinical } \\
\text { characteristics for PMC. }\end{array}$ & Ila & C \\
\hline $\begin{array}{l}\text { PMC should be considered in asymptomatic } \\
\text { patients without unfavourable clinical and } \\
\text { anatomical characteristics }{ }^{c} \text { for PMC and: } \\
\text { - high thromboembolic risk (history of } \\
\text { systemic embolism, dense spontaneous } \\
\text { contrast in the LA, new-onset or parox- } \\
\begin{array}{l}\text { ysmal atrial fibrillation), and/or } \\
\text { high risk of haemodynamic decompensa- } \\
\text { tion (systolic pulmonary pressure } \\
>50 \text { mmHg at rest, need for major non- } \\
\text { cardiac surgery, desire for pregnancy). }\end{array}\end{array}$ & Ila & C \\
\hline
\end{tabular}

$\mathrm{LA}=$ left atrium; $\mathrm{PMC}=$ percutaneous mitral commissurotomy

${ }^{\mathrm{a}}$ Class of recommendation.

'Level of evidence.

'Unfavourable characteristics for PMC can be defined by the presence of several of the following characteristics. Clinical characteristics: old age, history of commissurotomy, New York Heart Association class IV, permanent atrial fibrillation, severe pulmonary hypertension. Anatomical characteristics: echocardiographic score $>8$, Cormier score 3 (calcification of mitral valve of any extent as assessed by fluoroscopy), very small mitral valve area, severe tricuspid regurgitation. For the definition of scores see Table 9.

\subsection{Medical therapy}

Diuretics, beta-blockers, digoxin or heart rate-regulating calcium channel blockers can transiently improve symptoms. Anticoagulation with a target international normalized ratio (INR) between 2 and 3 is indicated in patients with either new-onset or paroxysmal atrial fibrillation.

In patients in sinus rhythm, oral anticoagulation is indicated when there has been a history of systemic embolism or a thrombus is present in the LA (recommendation class I, level of evidence C) and should also be considered when TOE shows dense spontaneous echocardiographic contrast or an enlarged LA (M-mode diameter $>50 \mathrm{~mm}$ or LA volume $>60 \mathrm{~mL} / \mathrm{m}^{2}$ ) (recommendation class lla, level of evidence $\mathrm{C}$ ). Patients with moderate to severe mitral stenosis and 


\section{Table 8 Contra-indications for percutaneous mitral commissurotomy (PMC) ${ }^{\mathrm{a}}$}

\begin{tabular}{|l|}
\hline Contra-indications \\
\hline Mitral valve area $>1.5 \mathrm{~cm}^{2 \mathrm{a}}$ \\
\hline Left atrial thrombus \\
\hline More than mild mitral regurgitation \\
\hline Severe or bi-commissural calcification \\
\hline Absence of commissural fusion \\
\hline $\begin{array}{l}\text { Severe concomitant aortic valve disease, or severe combined } \\
\text { tricuspid stenosis and regurgitation requiring surgery }\end{array}$ \\
\hline Concomitant CAD requiring bypass surgery \\
\hline
\end{tabular}

$C A D=$ coronary artery disease

${ }^{\text {a }} \mathrm{PMC}$ may be considered in patients with valve area $>1.5 \mathrm{~cm}^{2}$ with symptoms that cannot be explained by another cause and if the anatomy is favourable. persistent atrial fibrillation should be kept on vitamin $\mathrm{K}$ antagonist (VKA) treatment and not receive NOACs.

Cardioversion is not indicated before intervention in patients with severe mitral stenosis, as it does not durably restore sinus rhythm. If atrial fibrillation is of recent onset and the LA is only moderately enlarged, cardioversion should be performed soon after successful intervention.

\subsection{Serial testing}

Asymptomatic patients with clinically significant mitral stenosis who have not undergone intervention should be followed up yearly by means of clinical and echocardiographic examinations and at longer intervals (2-3 years) in case of moderate stenosis.

Management of patients after successful PMC is similar to that of asymptomatic patients. Follow-up should be more frequent if asymptomatic restenosis occurs. When PMC is not successful, surgery should be considered early unless there are definite contraindications.

\section{Table 9 Echo scores: Wilkins score, ${ }^{145}$ Cormier score, ${ }^{150}$ and Echo Score "Revisited" for immediate outcome prediction $^{146}$}

\begin{tabular}{|c|c|c|c|c|c|}
\hline Grade & Mobility & \multicolumn{2}{|l|}{ Thickening } & Calcification & Subvalvular thickening \\
\hline I & $\begin{array}{l}\text { Highly mobile valve with only } \\
\text { leaflet tips restricted }\end{array}$ & \multicolumn{2}{|c|}{$\begin{array}{l}\text { Leaflets near normal in thickness } \\
(4-5 \mathrm{~mm})\end{array}$} & $\begin{array}{l}\text { A single area of increased echo } \\
\text { brightness }\end{array}$ & $\begin{array}{l}\text { Minimal thickening just below the } \\
\text { mitral leaflets }\end{array}$ \\
\hline 2 & $\begin{array}{l}\text { Leaflet mid and base portions have } \\
\text { normal mobility }\end{array}$ & \multicolumn{2}{|c|}{$\begin{array}{l}\text { Mid leaflets normal, considerable } \\
\text { thickening of margins }(5-8 \mathrm{~mm})\end{array}$} & $\begin{array}{l}\text { Scattered areas of brightness } \\
\text { confined to leaflet margins }\end{array}$ & $\begin{array}{l}\text { Thickening of chordal structures } \\
\text { extending to one third of the } \\
\text { chordal length }\end{array}$ \\
\hline 3 & $\begin{array}{l}\text { Valve continues to move forward } \\
\text { in diastole, mainly from the base }\end{array}$ & \multicolumn{2}{|c|}{$\begin{array}{l}\text { Thickening extending through the } \\
\text { entire leaflet }(5-8 \mathrm{~mm})\end{array}$} & $\begin{array}{l}\text { Brightness extending into the } \\
\text { mid portions of the leaflets }\end{array}$ & $\begin{array}{l}\text { Thickening extended to distal third } \\
\text { of the chords }\end{array}$ \\
\hline 4 & $\begin{array}{l}\text { No or minimal forward movement } \\
\text { of the leaflets in diastole }\end{array}$ & \multicolumn{2}{|l|}{$\begin{array}{l}\text { Considerable thickening of all } \\
\text { leaflet tissue }(>8-10 \mathrm{~mm})\end{array}$} & $\begin{array}{l}\text { Extensive brightness throughout } \\
\text { much of the leaflet tissue }\end{array}$ & $\begin{array}{l}\text { Extensive thickening and shortening } \\
\text { of all chordal structures extending } \\
\text { down to the papillary muscles }\end{array}$ \\
\hline \multicolumn{6}{|c|}{ The total score is the sum of the four items and ranges between 4 and 16.} \\
\hline \multicolumn{3}{|c|}{ Echocardiographic group } & \multicolumn{3}{|c|}{ Mitral valve anatomy } \\
\hline \multicolumn{3}{|l|}{ Group I } & \multicolumn{3}{|c|}{$\begin{array}{l}\text { Pliable non-calcified anterior mitral leaflet and mild subvalvular disease } \\
\text { (i.e., thin chordae } \geq 10 \mathrm{~mm} \text { long) }\end{array}$} \\
\hline \multicolumn{3}{|l|}{ Group 2} & \multicolumn{3}{|c|}{$\begin{array}{l}\text { Pliable non-calcified anterior mitral leaflet and severe subvalvular disease } \\
\text { (i.e., thickened chordae }<10 \mathrm{~mm} \text { long) }\end{array}$} \\
\hline \multicolumn{2}{|l|}{ Group 3} & & \multicolumn{3}{|c|}{$\begin{array}{l}\text { Calcification of mitral valve of any extent, as assessed by fluoroscopy, } \\
\text { whatever the state of subvalvular apparatus }\end{array}$} \\
\hline \multicolumn{6}{|c|}{ Echo Score "Revisited" for immediate outcome prediction"146 } \\
\hline \multicolumn{3}{|c|}{ Echocardiographic variables } & \multicolumn{3}{|c|}{ Points for score (0 to II) } \\
\hline \multicolumn{3}{|c|}{ Mitral valve area $\leq \mathrm{lcm}^{2}$} & \multicolumn{3}{|l|}{2} \\
\hline \multicolumn{3}{|c|}{ Maximum leaflet displacement $\leq 12 \mathrm{~mm}$} & \multicolumn{3}{|l|}{3} \\
\hline \multicolumn{3}{|c|}{ Commissural area ratio $\geq 1.25$} & \multicolumn{3}{|l|}{3} \\
\hline
\end{tabular}

Risk groups for Echo score "Revisited": low (score 0 - 3); intermediate (score 4 - 5); high (score 6 - 11) 


\subsection{Special patient populations}

When restenosis with symptoms occurs after surgical commissurotomy or PMC, reintervention in most cases requires valve replacement, but PMC can be proposed in selected candidates with favourable characteristics if the predominant mechanism is commissural refusion. ${ }^{151}$

In the elderly population with rheumatic mitral stenosis when surgery is high risk, PMC is a useful option, even if only palliative. In other elderly patients, surgery is preferable. ${ }^{146,148,149}$ However, in elderly patients with degenerative mitral stenosis with severely calcified mitral annulus, surgery is very high risk. As there is no commissural fusion in these cases, degenerative mitral stenosis is not amenable to PMC. ${ }^{143}$ If degenerative mitral stenosis is severe, very preliminary experience has suggested that transcatheter valve implantation of a TAVI bioprosthesis in the mitral position is feasible in symptomatic elderly patients who are inoperable if the anatomy is suitable. ${ }^{152}$

In patients with severe mitral stenosis combined with severe aortic valve disease, surgery is preferable when it is not contraindicated. The management of patients in whom surgery is contraindicated is difficult and requires a comprehensive and individualized evaluation by the Heart Team.

In cases with severe mitral stenosis with moderate aortic valve disease, PMC can be performed to postpone the surgical treatment of both valves.

In patients with severe tricuspid regurgitation, PMC may be considered in selected patients with sinus rhythm, moderate atrial enlargement and functional tricuspid regurgitation secondary to pulmonary hypertension. In other cases, surgery on both valves is preferred. ${ }^{153}$

Valve replacement is the only option for the treatment of rare cases of severe mitral stenosis of non-rheumatic origin where commissural fusion is absent.

\section{Key points}

- Most patients with severe mitral stenosis and favourable valve anatomy currently undergo PMC.

- Decision making as to the type of intervention in patients with unfavourable anatomy is still a matter of debate and must take into account the multifactorial nature of predicting the results of PMC.

\section{Gaps in evidence}

- The scores predicting the results and complications of PMC, particularly those of severe mitral regurgitation, must be refined.

- The potential role of transcatheter mitral valve implantation in high-risk patients is to be determined, particularly those with severe degenerative mitral stenosis.

\section{Tricuspid regurgitation}

Pathological tricuspid regurgitation is more often secondary, due to RV dysfunction following pressure and/or volume overload in the presence of structurally normal leaflets. ${ }^{2}$ Possible causes of primary tricuspid regurgitation are infective endocarditis (especially in intravenous drug addicts), ${ }^{154}$ rheumatic heart disease, carcinoid syndrome, myxomatous disease, endomyocardial fibrosis, Ebstein's anomaly and congenitally dysplastic valves, drug-induced valve diseases, thoracic trauma and iatrogenic valve damage.

\subsection{Evaluation}

Echocardiography is the ideal technique to evaluate tricuspid regurgitation. In primary tricuspid regurgitation, the aetiology can usually be identified from specific abnormalities of the valve structure. ${ }^{28,115}$ In secondary tricuspid regurgitation, the degree of dilatation of the annulus, the RV dimension and function and the degree of tricuspid valve deformation should be measured. ${ }^{2}$ Evaluation of tricuspid regurgitation severity (integration of multiple qualitative and quantitative parameters) and pulmonary systolic pressure should be carried out as currently recommended (Table 4). ${ }^{2}$ It has to be noted that the problem of elevated pulmonary vascular resistance may be disguised in the presence of severe tricuspid regurgitation since its velocity may be lower than expected in the case of pulmonary hypertension.

Evaluations of RV dimensions and function should be conducted despite the existing limitations of current indices of RV function. ${ }^{53}$ The presence of associated lesions (looking carefully at the associated valve lesions, particularly on the left side) and LV function should be assessed.

In experienced laboratories, 3D measurements of $R V$ volumes can be considered, which may be similar to those obtained by CMR. ${ }^{155}$ However, when available, CMR is the preferred method for evaluating RV size and function and represents the gold standard for assessing RV volumes and function. ${ }^{155}$

Cardiac catheterization is not needed to diagnose tricuspid regurgitation or estimate its severity but should be obtained in patients in whom isolated tricuspid valve surgery is contemplated for secondary tricuspid regurgitation to evaluate haemodynamics, in particular pulmonary vascular resistance.

\subsection{Indications for intervention}

The timing of surgical intervention remains controversial, mostly due to the limited data available and their heterogeneous nature (see table of recommendations for indications for tricuspid valve surgery and Figure 6). ${ }^{156-160}$ Surgery should be carried out sufficiently early to avoid irreversible RV dysfunction.

In severe primary tricuspid regurgitation, surgery is not only recommended in symptomatic patients but should also be considered in asymptomatic patients when progressive RV dilatation or decline of $\mathrm{RV}$ function is observed. Although these patients respond well to diuretic therapy, delaying surgery is likely to result in irreversible RV damage, organ failure and poor results of late surgical intervention.

In secondary tricuspid regurgitation, adding a tricuspid repair, if indicated, during left-sided surgery does not increase operative risk and has been demonstrated to provide reverse remodelling of the $\mathrm{RV}$ and improvement of functional status even in the absence of substantial tricuspid regurgitation when annulus dilatation is present. ${ }^{156,157,160}$ It should therefore be performed liberally. Reoperation on the tricuspid valve in cases of persistent tricuspid regurgitation after mitral valve surgery carries a high risk, mostly due to the late referral and the consequently poor clinical condition of patients. To improve the prognosis of patients in this challenging scenario, the treatment of severe late tricuspid regurgitation following left-sided valve surgery should be considered earlier, even in asymptomatic patients, if there are signs of progressive RV dilatation or 


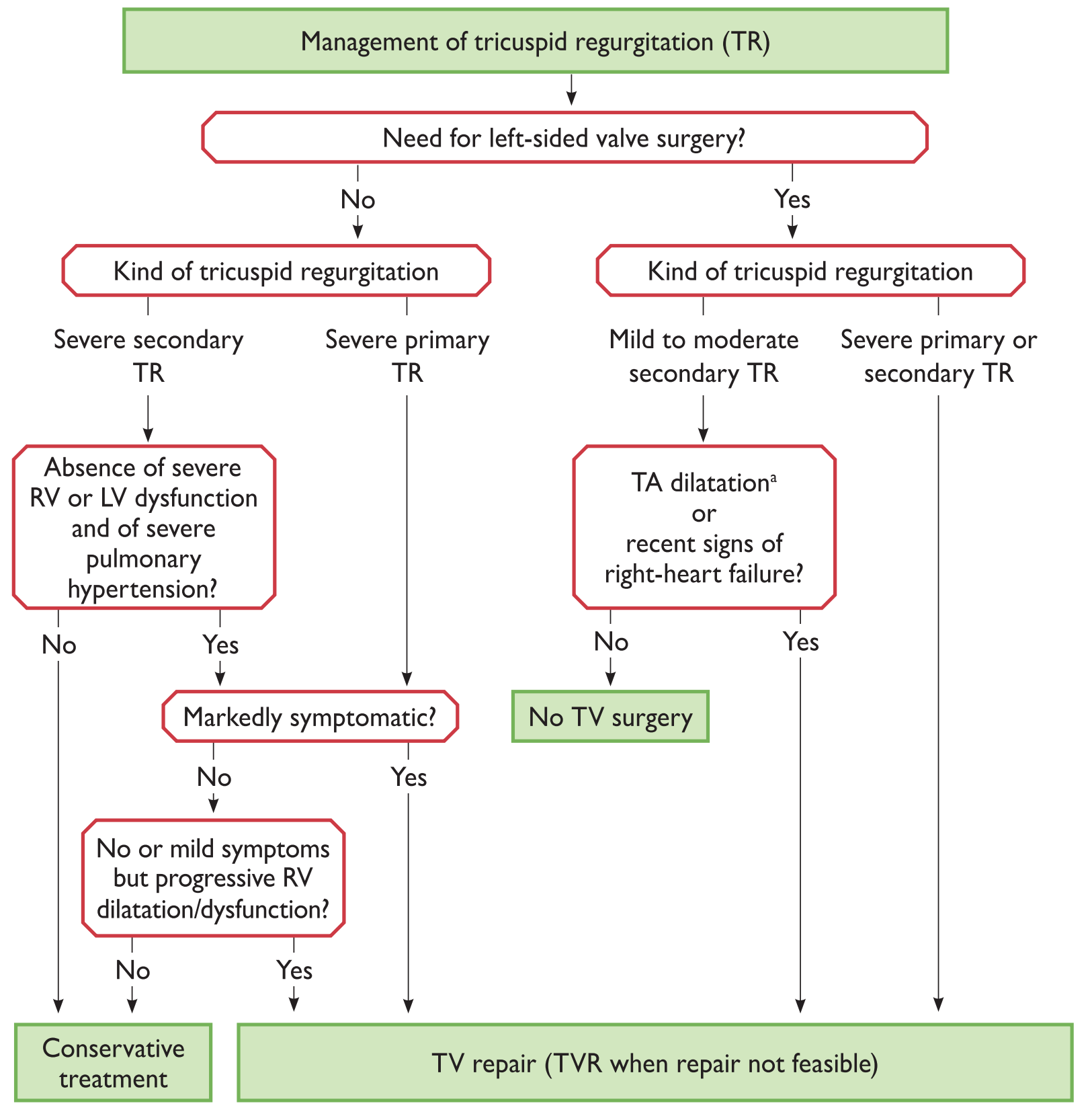

Figure 6 Indications for surgery in tricuspid regurgitation. $L V=$ left ventricular; $R V=$ right ventricular; $T A=$ tricuspid annulus; $T R=$ tricuspid regurgitation; TV = tricuspid valve; TVR = tricuspid valve replacement.

${ }^{\mathrm{a}} \mathrm{TA} \geq 40 \mathrm{~mm}$ or $>21 \mathrm{~mm} / \mathrm{m}^{2}$.

decline in RV function and in the absence of left-sided valve dysfunction, severe RV or LV dysfunction and severe pulmonary vascular disease/hypertension.

If possible, valve repair is preferable to valve replacement. Ring annuloplasty, preferably with prosthetic rings, is key to surgery for secondary tricuspid regurgitation. ${ }^{156,161}$ Valve replacement should be considered when the tricuspid valve leaflets are significantly tethered and the annulus is severely dilated. In the presence of transtricuspid pacemaker leads, the technique used should be adapted to the patient's condition and the surgeon's experience. Percutaneous repair techniques are in their infancy and must be further evaluated before any recommendations can be made. 


\section{Indications for tricuspid valve surgery}

\begin{tabular}{|c|c|c|}
\hline Recommendations & Class $^{\mathrm{a}}$ & Level $^{\mathrm{b}}$ \\
\hline \multicolumn{3}{|l|}{ Recommendations on tricuspid stenosis } \\
\hline $\begin{array}{l}\text { Surgery is indicated in symptomatic patients } \\
\text { with severe tricuspid stenosis. }\end{array}$ & I & c \\
\hline $\begin{array}{l}\text { Surgery is indicated in patients with severe } \\
\text { tricuspid stenosis undergoing left-sided } \\
\text { valve intervention. }{ }^{d}\end{array}$ & I & C \\
\hline \multicolumn{3}{|c|}{ Recommendations on primary tricuspid regurgitation } \\
\hline $\begin{array}{l}\text { Surgery is indicated in patients with severe } \\
\text { primary tricuspid regurgitation undergoing } \\
\text { left-sided valve surgery. }\end{array}$ & $\mathbf{I}$ & c \\
\hline $\begin{array}{l}\text { Surgery is indicated in symptomatic patients } \\
\text { with severe isolated primary tricuspid regur- } \\
\text { gitation without severe RV dysfunction. }\end{array}$ & I & c \\
\hline $\begin{array}{l}\text { Surgery should be considered in patients } \\
\text { with moderate primary tricuspid regurgita- } \\
\text { tion undergoing left-sided valve surgery. }\end{array}$ & Ila & C \\
\hline $\begin{array}{l}\text { Surgery should be considered in asympto- } \\
\text { matic or mildly symptomatic patients with } \\
\text { severe isolated primary tricuspid regurgita- } \\
\text { tion and progressive RV dilatation or deteri- } \\
\text { oration of RV function. }\end{array}$ & Ila & c \\
\hline \multicolumn{3}{|c|}{ Recommendations on secondary tricuspid regurgitation } \\
\hline $\begin{array}{l}\text { Surgery is indicated in patients with severe } \\
\text { secondary tricuspid regurgitation under- } \\
\text { going left-sided valve surgery. }\end{array}$ & I & c \\
\hline $\begin{array}{l}\text { Surgery should be considered in patients } \\
\text { with mild or moderate secondary tricuspid } \\
\text { regurgitation with a dilated annulus } \\
\text { ( } \geq 40 \mathrm{~mm} \text { or }>21 \mathrm{~mm} / \mathrm{m}^{2} \text { by } 2 \mathrm{D} \text { echocar- } \\
\text { diography) undergoing left-sided valve } \\
\text { surgery. }\end{array}$ & Ila & C \\
\hline $\begin{array}{l}\text { Surgery may be considered in patients } \\
\text { undergoing left-sided valve surgery with } \\
\text { mild or moderate secondary tricuspid } \\
\text { regurgitation even in the absence of annular } \\
\text { dilatation when previous recent right-heart } \\
\text { failure has been documented. }\end{array}$ & llb & c \\
\hline $\begin{array}{l}\text { After previous left-sided surgery and in } \\
\text { absence of recurrent left-sided valve dysfunc- } \\
\text { tion, surgery should be considered in patients } \\
\text { with severe tricuspid regurgitation who are } \\
\text { symptomatic or have progressive RV dilata- } \\
\text { tion/dysfunction, in the absence of severe RV } \\
\text { or LV dysfunction and severe pulmonary vas- } \\
\text { cular disease/hypertension. }\end{array}$ & Ila & C \\
\hline
\end{tabular}

$2 \mathrm{D}=$ two-dimensional; $\mathrm{LV}=$ left ventricular; $\mathrm{PMC}=$ percutaneous mitral commissurotomy; RV = right ventricular.

${ }^{\mathrm{a} C}$ Class of recommendation.

bLevel of evidence.

'Percutaneous balloon valvuloplasty can be attempted as a first approach if tricuspid stenosis is isolated.

dPercutaneous balloon valvuloplasty can be attempted if PMC can be performed on the mitral valve.

\section{Tricuspid stenosis}

Tricuspid stenosis is often combined with tricuspid regurgitation, most frequently of rheumatic origin. It is therefore almost always associated with left-sided valve lesions, particularly mitral stenosis, that usually dominate the clinical presentation. Other causes are rare, including congenital, drug-induced valve diseases, Whipple's disease, endocarditis and large right atrial tumour.

\subsection{Evaluation}

Echocardiography provides the most useful information. Tricuspid stenosis is often overlooked and requires careful evaluation. Echocardiographic evaluation of the anatomy of the valve and its subvalvular apparatus is important to assess valve reparability. No generally accepted grading of tricuspid stenosis severity exists, but a mean gradient $\geq 5 \mathrm{mmHg}$ at normal heart rate is considered indicative of clinically significant tricuspid stenosis. ${ }^{3}$ Catheterization is no longer used for evaluating the severity of tricuspid stenosis.

\subsection{Indications for intervention}

The lack of pliable leaflet tissue is the main limitation for valve repair. Even though this is still a matter of debate, biological prostheses for valve replacement are usually preferred over mechanical ones because of the high risk of thrombosis carried by the latter and the satisfactory long-term durability of the former in the tricuspid position. $^{162}$

Percutaneous balloon tricuspid dilatation has been performed in a limited number of cases, either alone or alongside PMC, but frequently induces significant regurgitation. There is a lack of data on long-term results. ${ }^{163}$

Intervention on the tricuspid valve is usually carried out at the time of intervention on the other valves in patients who are symptomatic despite medical therapy. The choice between repair or valve replacement depends on valve anatomy and surgical expertise. Balloon commissurotomy can be considered in the rare cases with anatomically suitable valves when tricuspid stenosis is isolated, or additional mitral stenosis can also be treated interventionally (see table of recommendations in section 7.2 listing indications for PMC and mitral valve surgery in clinically significant mitral stenosis).

\subsection{Medical therapy}

Diuretics are useful in the presence of heart failure but are of limited long-term efficacy.

\section{Key points}

- Tricuspid stenosis is a rare condition, whereas tricuspid regurgitation is more common, especially in its secondary form.

- For appropriate management, secondary tricuspid regurgitation has to be clearly distinguished from primary tricuspid regurgitation. 
- Similar to mitral regurgitation, primary tricuspid regurgitation requires intervention sufficiently early to avoid secondary damage of the RV, which is associated with poor outcome.

- Secondary tricuspid regurgitation should be liberally treated at the time of left-sided valve surgery.

- Consideration of isolated surgery of secondary tricuspid regurgitation after previous left-sided valve surgery requires comprehensive assessment of the underlying disease, pulmonary haemodynamics and RV function.

\section{Gaps in evidence}

- Criteria for optimal timing of surgery in primary tricuspid regurgitation require refinement.

- Criteria for concomitant tricuspid valve surgery at the time of left-sided surgery in patients without severe tricuspid valve disease require refinement.

- The potential role of transcatheter tricuspid valve treatment in high-risk patients needs to be determined.

\section{Combined and multiple-valve diseases}

Significant stenosis and regurgitation can be found on the same valve. Disease of multiple valves may be encountered in several conditions, particularly in rheumatic and congenital heart disease, but also less frequently in degenerative valve disease. There is a lack of data on combined or multiple-valve diseases. This does not allow for evidence-based recommendations. ${ }^{164}$ The general principles for the management of combined or multiple-valve disease are as follows:

- When either stenosis or regurgitation is predominant, management follows the recommendations concerning the predominant $\mathrm{VHD}$. When the severity of both stenosis and regurgitation is balanced, indications for interventions should be based on symptoms and objective consequences rather than on the indices of severity of stenosis or regurgitation. In this setting, consideration of the pressure gradient that reflects the haemodynamic burden of the valve lesion becomes more important than valve area and measures of the regurgitation for the assessment of disease severity.

- Besides the separate assessment of each valve lesion, it is necessary to take into account the interaction between the different valve lesions. As an illustration, associated mitral regurgitation may lead to underestimation of the severity of aortic stenosis, as decreased stroke volume due to mitral regurgitation lowers the flow across the aortic valve and hence the aortic gradient. This underlines the need to combine different measurements, including assessment of valve areas, if possible using methods that are less dependent on loading conditions, such as planimetry.

- Indications for intervention are based on global assessment of the consequences of the different valve lesions (i.e. symptoms or presence of LV dilatation or dysfunction). Intervention can be considered for non-severe multiple lesions associated with symptoms or leading to LV impairment.

- The decision to intervene on multiple valves should take into account the extra surgical risk of combined procedures.

- The choice of surgical technique should take into account the presence of the other VHD; repair remains the ideal option.

The management of specific associations of VHD is detailed in the individual sections of this document.

\section{Key points}

- In combined VHD, pathology is considered severe even if both stenosis and regurgitation are only of moderate severity and pressure gradients become of major importance for assessment.

- Management of multiple valve disease is dictated by the predominant VHD.

\section{Gaps in evidence}

- More data on the natural history and the impact of intervention on outcome are required to better define the indications for intervention.

\section{Prosthetic valves}

Every valve prosthesis introduces a new disease process. In practice, the choice is between a mechanical and a biological prosthesis. Randomized trials comparing both prostheses consistently found similar survival, no significant difference in rates of valve thrombosis and thromboembolism, higher rates of bleeding with mechanical prostheses and higher rates of reintervention with bioprostheses. $^{165-167}$

\subsection{Choice of prosthetic valve}

The choice between a mechanical and a biological valve in adults is determined mainly by estimating the risk of anticoagulation-related bleeding and thromboembolism with a mechanical valve versus the risk of structural valve deterioration with a bioprosthesis and by considering the patient's lifestyle and preferences. Rather than setting arbitrary age limits, prosthesis choice should be discussed in detail with the informed patient, cardiologists and surgeons, taking into account the factors detailed below (see tables of recommendations in section 11.1). Bioprostheses should be considered in patients whose life expectancy is lower than the presumed durability of the bioprosthesis, particularly if comorbidities may necessitate further surgical procedures, and in those with increased bleeding risk. In women who wish to become pregnant, the high risk of thromboembolic complications with a mechanical prosthesis during pregnancy and the low risk of elective reoperation are incentives to consider a bioprosthesis, despite the rapid occurrence of structural valve deterioration in this age group. 


\section{Choice of the aortic/mitral prosthesis in favour of a mechanical prosthesis; the decision is based on the integration of} several of the following factors

\begin{tabular}{|c|c|c|}
\hline Recommendations & Class $^{\mathbf{a}}$ & Level $^{\mathbf{b}}$ \\
\hline $\begin{array}{l}\text { A mechanical prosthesis is recommended according to the desire of the informed patient and if there are no contraindi- } \\
\text { cations to long-term anticoagulation. }{ }^{c}\end{array}$ & $\mathbf{I}$ & c \\
\hline A mechanical prosthesis is recommended in patients at risk of accelerated structural valve deterioration. ${ }^{d}$ & $\mathbf{I}$ & c \\
\hline $\begin{array}{l}\text { A mechanical prosthesis should be considered in patients already on anticoagulation because of a mechanical prosthesis } \\
\text { in another valve position. }\end{array}$ & Ila & C \\
\hline $\begin{array}{l}\text { A mechanical prosthesis should be considered in patients }<60 \text { years of age for prostheses in the aortic position and } \\
<65 \text { years of age for prostheses in the mitral position. }\end{array}$ & Ila & C \\
\hline $\begin{array}{l}\text { A mechanical prosthesis should be considered in patients with a reasonable life expectancy }{ }^{f} \text { for whom future redo valve } \\
\text { surgery would be at high risk. }\end{array}$ & Ila & C \\
\hline $\begin{array}{l}\text { A mechanical prosthesis may be considered in patients already on long-term anticoagulation due to the high risk for } \\
\text { thromboembolism. }\end{array}$ & Illb & c \\
\hline
\end{tabular}

$\mathrm{LV}=$ left ventricular.

${ }^{\mathrm{a}}$ Class of recommendation.

'Level of evidence.

Increased bleeding risk because of comorbidities, compliance concerns or geographic, lifestyle or occupational conditions.

'Young age (<40 years), hyperparathyroidism.

en patients 60-65 years of age who should receive an aortic prosthesis and those between 65 and 70 years of age in the case of mitral prosthesis, both valves are acceptable and the choice requires careful analysis of factors other than age.

${ }_{\mathrm{f}}^{\mathrm{L}}$ ife expectancy should be estimated at $>10$ years according to age, sex, comorbidities and country-specific life expectancy.

${ }^{g}$ Risk factors for thromboembolism are atrial fibrillation, previous thromboembolism, hypercoagulable state and severe LV systolic dysfunction.

\section{Choice of the aortic/mitral prosthesis in favour of a bioprosthesis; the decision is based on the integration of several of the following factors}

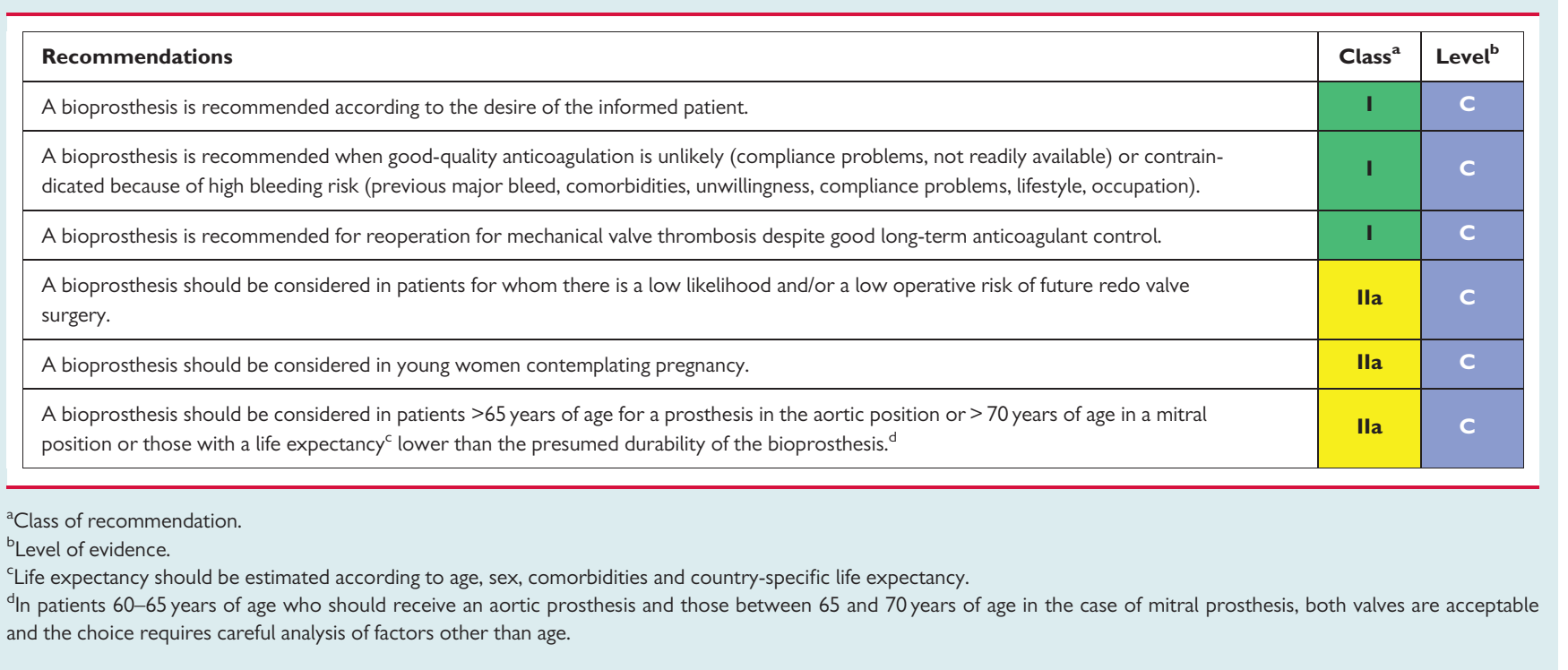

\subsection{Management after valve intervention}

Thromboembolism and anticoagulant-related bleeding present the majority of complications experienced by prosthetic valve recipients. Endocarditis prophylaxis and management of prosthetic valve endocarditis are detailed in a separate ESC guideline. ${ }^{28}$

\subsubsection{Baseline assessment and modalities of follow-up} All patients require lifelong follow-up by a cardiologist after valve surgery to detect early deterioration in prosthetic function or ventricular function or progressive disease of another heart valve. Clinical assessment should be performed yearly or as soon as possible if new cardiac symptoms occur. TTE should be performed if any new symptoms 
occur after valve replacement or if complications are suspected. After transcatheter as well as surgical implantation of a bioprosthetic valve, echocardiography, including the measurement of transprosthetic gradients, should be performed within 30 days (preferably $\sim 30$ days for surgery) after valve implantation (i.e. baseline imaging), at 1 year after implantation and annually thereafter. ${ }^{168}$ TOE should be considered if TTE is of poor quality and in all cases of suspected prosthetic dysfunction or endocarditis. ${ }^{169,170}$ Cinefluoroscopy for mechanical valves and MSCT scanning provide useful additional information if valve thrombus or pannus are suspected to impair valve function. ${ }^{170}$

\subsubsection{Antithrombotic management}

\subsubsection{General management}

Antithrombotic management should address effective control of modifiable risk factors for thromboembolism in addition to the prescription of antithrombotic drugs. ${ }^{171}$ Indications for antithrombotic therapy after valve repair or replacement are summarized in the table of recommendations for indications for antithrombotic therapy after valvular surgery.

In patients with surgical aortic bioprostheses, the use of low-dose aspirin is now favoured as an alternative to postoperative anticoagulant therapy, although this relies on low-level evidence. ${ }^{42,172,173}$

When postoperative anticoagulant therapy is indicated, oral anticoagulation should be started during the first postoperative days. Intravenous unfractionated heparin (UFH), monitored to an activated partial thromboplastin time of 1.5-2.0 times the control value, enables rapid anticoagulation to be obtained before the INR rises. ${ }^{42}$ Low-molecular-weight heparin (LMWH) seems to offer effective and stable anticoagulation and has been used in observational series mostly using enoxaparin. ${ }^{174,175}$ This is off-label use.

The first postoperative month is a high-risk period for thromboembolism. The addition of aspirin to anticoagulant therapy decreases postoperative thromboembolic risk but increases bleeding risk and cannot be recommended routinely. ${ }^{176}$

VKAs should be favoured when long-term anticoagulant therapy is needed in patients with a bioprosthesis. Despite the absence of data from clinical trials, NOACs can be used in patients who have atrial fibrillation associated with a bioprosthesis after the third postoperative month. ${ }^{43}$ There is no evidence to support the use of antiplatelet agents beyond 3 months in patients with surgical bioprostheses who do not have an indication other than the presence of the bioprosthesis itself.

A combination of low-dose aspirin and a thienopyridine is commonly used early after TAVI, followed by aspirin or a thienopyridine alone in patients who have no other indication for oral anticoagulation. Recent data suggest that single antiplatelet therapy may have a better safety profile than dual antiplatelet therapy after TAVI. ${ }^{177}$ Observational findings suggest that anticoagulant therapy reduces the incidence of subclinical thrombosis compared with dual antiplatelet therapy. ${ }^{178}$ The results of ongoing large-scale, dedicated trials are needed to improve evidence in this field.

\subsubsection{Target international normalized ratio}

Target INR should be adapted to patient risk factors and the thrombogenicity of the prosthesis (Table 10). ${ }^{171}$ Recent randomized trials supported lower target INRs for aortic prostheses. ${ }^{186-188}$ However, limited statistical power, certain methodological concerns and the
Table 10 Target INR for mechanical prostheses

\begin{tabular}{|c|c|c|}
\hline \multirow{2}{*}{$\begin{array}{l}\text { Prosthesis } \\
\text { thrombogenicity }\end{array}$} & \multicolumn{2}{|c|}{ Patient-related risk factors ${ }^{a}$} \\
\hline & None & $\geq \mathrm{l}$ risk factor \\
\hline Low $^{b}$ & 2.5 & 3.0 \\
\hline Medium ${ }^{c}$ & 3.0 & 3.5 \\
\hline High $^{d}$ & 3.5 & 4.0 \\
\hline \multicolumn{3}{|c|}{ 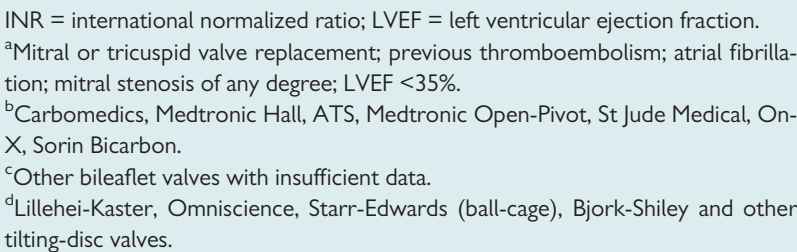 } \\
\hline
\end{tabular}

restriction to certain prostheses and/or to the use of INR selfmanagement led the Task Force not to change recommendations for target INR.

We recommend a median INR value rather than a range to avoid considering extreme values in the target range as a valid target INR. High variability of the INR is a strong independent predictor of reduced survival after valve replacement. There is now evidence that INR self-management reduces INR variability and clinical events, including patients with heart valve prosthesis ${ }^{181}$; however, appropriate training and regular quality control are required. However, monitoring by an anticoagulant clinic should be considered for patients with unstable INR or anticoagulant-related complications. Systematic genotyping of patients on VKA treatment is not recommended in the absence of convincing clinical benefit and concerns regarding costeffectiveness. $^{189}$

\subsubsection{Management of vitamin K antagonist overdose and bleeding}

The risk of major bleeding rises considerably when the INR exceeds 4.5 and increases exponentially above an INR of 6.0. An INR $\geq 6.0$ therefore requires rapid reversal of anticoagulation because of the risk of subsequent bleeding.

In the absence of bleeding, management depends on the target INR, the actual INR and the half-life of the VKA used. It is possible to stop oral anticoagulation and to allow the INR to fall gradually or to give oral vitamin $\mathrm{K}$ in increments of 1 or $2 \mathrm{mg} .{ }^{190}$ Immediate reversal of anticoagulation using intravenous prothrombin complex concentrate and vitamin $\mathrm{K}$ is required only for severe bleeding, defined as not amenable to local control, threatening life or important organ function (e.g. intracranial bleeding), causing haemodynamic instability or requiring an emergency surgical procedure or transfusion. ${ }^{190}$ There are no data suggesting that the risk of thromboembolism due to transient reversal of anticoagulation outweighs the consequences of severe bleeding in patients with mechanical prostheses. The optimal time to restart anticoagulant therapy should be discussed in relation to the location of the bleeding event, its evolution and interventions performed to stop bleeding and/or to treat an underlying cause. $^{191}$ 


\section{Indications for antithrombotic therapy in patients with a prosthetic heart valve or valve repair}

\begin{tabular}{|c|c|c|}
\hline Recommendations & Class $^{\mathrm{a}}$ & Level $^{\mathrm{b}}$ \\
\hline \multicolumn{3}{|l|}{ Mechanical prostheses } \\
\hline Oral anticoagulation using a VKA is recommended lifelong for all patients. ${ }^{179,180}$ & I & B \\
\hline $\begin{array}{l}\text { Bridging using therapeutic doses of UFH or LMWH is recommended when VKA treatment should be } \\
\text { interrupted. }\end{array}$ & $\mathbf{I}$ & C \\
\hline $\begin{array}{l}\text { The addition of low-dose aspirin ( } 75-100 \mathrm{mg} / \text { day) to VKA should be considered after thromboembolism } \\
\text { despite an adequate INR. }\end{array}$ & Ila & C \\
\hline $\begin{array}{l}\text { The addition of low-dose aspirin ( } 75-100 \mathrm{mg} / \text { day) to VKA may be considered in the case of concomitant } \\
\text { atherosclerotic disease. }\end{array}$ & Illb & C \\
\hline INR self-management is recommended provided appropriate training and quality control are performed. ${ }^{181}$ & I & B \\
\hline $\begin{array}{l}\text { In patients treated with coronary stent implantation, triple therapy with aspirin }(75-100 \mathrm{mg} / \text { day }) \text {, clopidogrel } \\
(75 \mathrm{mg} / \text { day) and VKA should be considered for } 1 \text { month, irrespective of the type of stent used and the clinical } \\
\text { presentation (i.e. ACS or stable CAD). }{ }^{182}\end{array}$ & Ila & $\mathbf{B}$ \\
\hline $\begin{array}{l}\text { Triple therapy comprising aspirin }(75-100 \mathrm{mg} / \text { day }) \text {, clopidogrel }(75 \mathrm{mg} / \text { day }) \text { and VKA for }>1 \mathrm{month} \text { and up to } \\
6 \text { months should be considered in patients with high ischaemic risk due to ACS or other anatomical/procedural } \\
\text { characteristics that outweighs the bleeding risk. }{ }^{182}\end{array}$ & Ila & $\mathbf{B}$ \\
\hline $\begin{array}{l}\text { Dual therapy comprising VKA and clopidogrel }(75 \mathrm{mg} / \text { day) should be considered as an alternative to } 1 \text {-month } \\
\text { triple antithrombotic therapy in patients in whom the bleeding risk outweighs the ischaemic risk. }{ }^{183,184}\end{array}$ & Ila & $\mathbf{A}$ \\
\hline $\begin{array}{l}\text { In patients who have undergone } \mathrm{PCl} \text {, discontinuation of antiplatelet treatment should be } \\
\text { considered at } 12 \text { months. }{ }^{185}\end{array}$ & Ila & B \\
\hline $\begin{array}{l}\text { In patients requiring aspirin and/or clopidogrel in addition to VKA, the dose intensity of VKA should be care- } \\
\text { fully regulated with a target INR in the lower part of the recommended target range and a time in the thera- } \\
\text { peutic range }>65-70 \% .{ }^{182,184}\end{array}$ & Ila & $\mathbf{B}$ \\
\hline The use of NOACs is contraindicated. ${ }^{45}$ & III & B \\
\hline \multicolumn{3}{|l|}{ Bioprostheses } \\
\hline $\begin{array}{l}\text { Oral anticoagulation is recommended lifelong for patients with surgical or transcatheter implanted biopros- } \\
\text { theses who have other indications for anticoagulation. }{ }^{c}\end{array}$ & I & C \\
\hline $\begin{array}{l}\text { Oral anticoagulation using a VKA should be considered for the first } 3 \text { months after surgical implantation of a } \\
\text { mitral or tricuspid bioprosthesis. }\end{array}$ & Ila & C \\
\hline $\begin{array}{l}\text { Oral anticoagulation using a VKA should be considered for the first } 3 \text { months after surgical mitral or tricuspid } \\
\text { valve repair. }\end{array}$ & Ila & C \\
\hline $\begin{array}{l}\text { Low-dose aspirin ( } 75 \text { - } 100 \mathrm{mg} / \text { day) should be considered for the first } 3 \text { months after surgical implantation of } \\
\text { an aortic bioprosthesis or valve-sparing aortic surgery. }\end{array}$ & Ila & C \\
\hline $\begin{array}{l}\text { Dual antiplatelet therapy should be considered for the first 3-6 months after TAVI, followed by lifelong single } \\
\text { antiplatelet therapy in patients who do not need oral anticoagulation for other reasons. }\end{array}$ & Ila & C \\
\hline Single antiplatelet therapy may be considered after TAVI in the case of high bleeding risk. & Illb & C \\
\hline $\begin{array}{l}\text { Oral anticoagulation may be considered for the first } 3 \text { months after surgical implantation of an aortic } \\
\text { bioprosthesis. }\end{array}$ & Ilb & C \\
\hline
\end{tabular}

$\mathrm{ACS}=$ acute coronary syndrome; CAD = coronary artery disease; INR = international normalized ratio; LMWH = low-molecular-weight heparin; LV = left ventricular; PCI = percutaneous coronary intervention; $\mathrm{NOAC}=$ non-vitamin $\mathrm{K}$ antagonist oral anticoagulant; $\mathrm{TAVI}=$ transcatheter aortic valve implantation; $\mathrm{UFH}=$ unfractionated heparin; $\mathrm{VKA}=$ vitamin $\mathrm{K}$ antagonist.

${ }^{\mathrm{a}}$ Class of recommendation.

bevel of evidence.

'Atrial fibrillation, venous thromboembolism, hypercoagulable state or, with a lesser degree of evidence, severely impaired LV dysfunction (ejection fraction <35\%). 


\section{Patients with a mechanical valve undergoing $\mathrm{PCl}$}

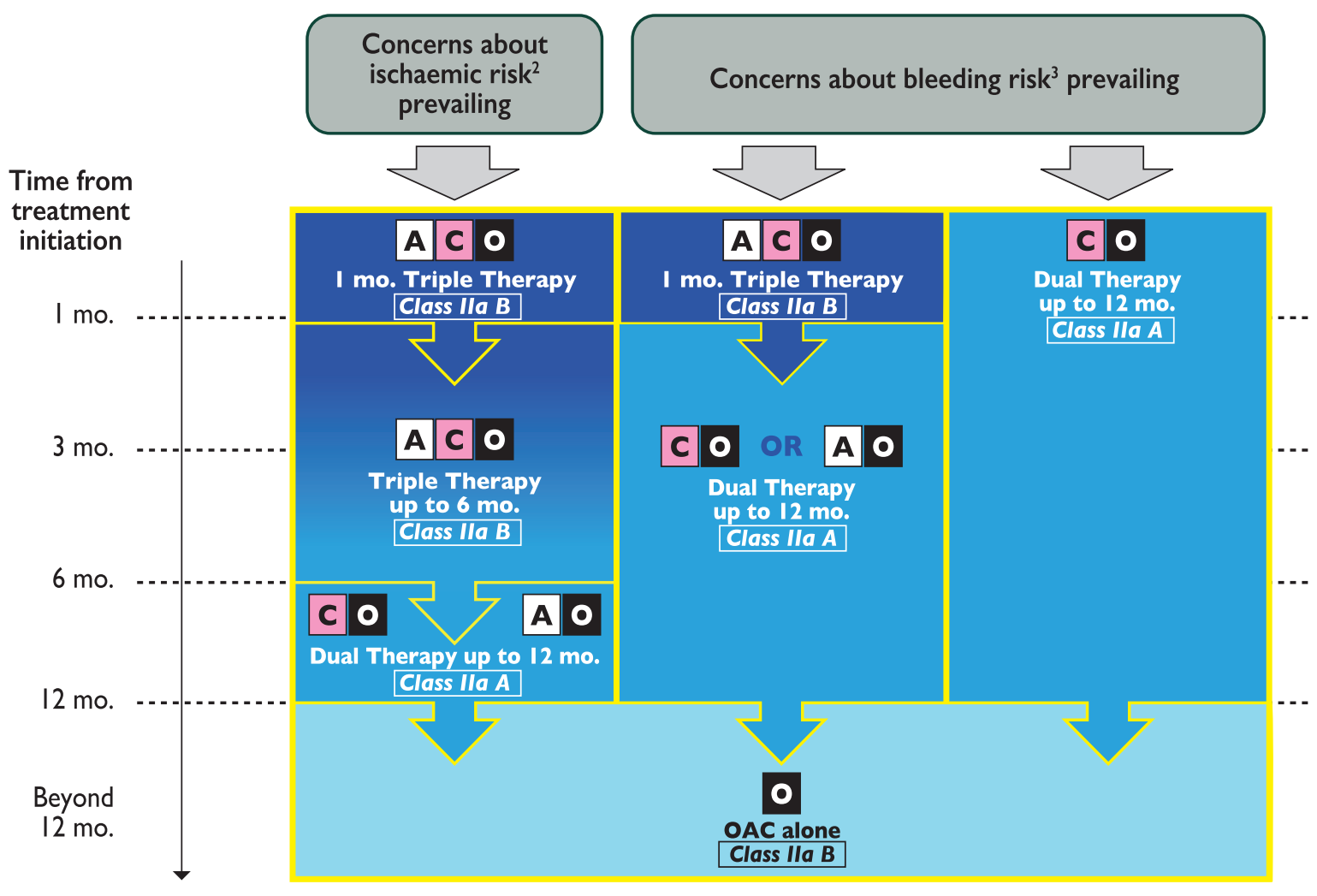
$\mathbf{A}=$ Aspirin
C $=$ Clopidogrel
- = Oral anticoagulation

1. Periprocedural administration of aspirin and clopidogrel during $\mathrm{PCl}$ is recommended irrespective of the treatment strategy.

2. High ischaemic risk is considered as an acute clinical presentation or

anatomical/procedural features which might increase the risk for myocardial infarction.

3. Bleeding risk can be estimated by HAS-BLED or ABC score

Figure 7 Antithrombotic therapy in patients with mechanical valve prosthesis undergoing PCl (adapted from the 2017 ESC Focused Update on Dual Antiplatelet Therapy ${ }^{195}$ ). $A=$ aspirin; $A B C=$ age, biomarkers, clinical history; $A C S=$ acute coronary syndrome; $C=$ clopidogrel; mo. $=$ month(s); $\mathrm{O}=$ oral anticoagulation with a vitamin $\mathrm{K}$ antagonist; $\mathrm{PCl}=$ percutaneous coronary intervention. For more details regarding estimation of bleeding risk (HAS-BLED and ABC score) see the 2017 ESC Focused Update on Dual Antiplatelet Therapy. ${ }^{195}$

11.2.2.4 Combination of oral anticoagulants with antiplatelet drugs The addition of aspirin with contemporary target INRs has not been studied in patients without vascular disease. ${ }^{42}$ Underlying uncertainties on the risk-benefit ratio of the combination of VKA with aspirin account for discrepancies between different recommendations. ${ }^{192,193}$ When added to anticoagulation, antiplatelet agents decrease thromboembolic risk but increase the risk of major bleeding. ${ }^{194}$ Therefore they should not be prescribed to all patients with prosthetic valves but should be reserved for specific indications according to the analysis of benefit and increased risk of major bleeding. If used, the lower recommended dose should be prescribed (e.g. aspirin 75 - $100 \mathrm{mg} /$ day).

Indications for the addition of an antiplatelet agent to oral anticoagulants are detailed in section 11.2.2.1 (see table of recommendations for indications for antithrombotic therapy in patients with a prosthetic heart valve or valve repair) and in Figure 7. The use of prasugrel or ticagrelor as part of triple therapy should be avoided. ${ }^{37}$ During triple antithrombotic therapy, close monitoring of INR is advised and INR should be kept in the low target range. 


\subsubsection{Interruption of anticoagulant therapy for planned invasive} procedures

Anticoagulation during non-cardiac surgery requires careful management based on risk assessment. ${ }^{196}$ It is recommended not to interrupt oral anticoagulation for most minor surgical procedures (including dental extraction, cataract removal) and those procedures where bleeding is easily controlled. ${ }^{197}$ Major surgical procedures require an INR $<1.5$. In patients with a mechanical prosthesis, oral anticoagulant therapy should be stopped before surgery and bridging using heparin is recommended. ${ }^{196}$ UFH remains the only approved heparin treatment in patients with mechanical prostheses; intravenous administration should be favoured over the subcutaneous route. The use of subcutaneous LMWH, although off-label, is an alternative to UFH for bridging. When LMWHs are used they should be administered twice a day using therapeutic doses, adapted to body weight and renal function and, if possible, with monitoring of anti-Xa activity with a target of $0.5-1.0 \mathrm{U} / \mathrm{mL}$. Fondaparinux should not be used for bridging in patients with mechanical prosthesis. Practical modalities of anticoagulation bridging are detailed in Figure 8.

If required, after a careful risk-benefit assessment, combined aspirin therapy should be discontinued 1 week before a non-cardiac procedure.

Oral anticoagulation can be continued at modified doses in the majority of patients who undergo cardiac catheterization, in particular using the radial approach. In patients who require transseptal catheterization for valvular interventions, direct LV puncture or pericardial drainage, oral anticoagulants should be stopped and bridging anticoagulation administered. ${ }^{171}$

In patients who have a subtherapeutic INR during routine monitoring, bridging with UFH or preferably LMWH in an outpatient setting is indicated until a therapeutic INR value is reached.

\subsubsection{Management of valve thrombosis}

Obstructive valve thrombosis should be suspected promptly in any patient with any type of prosthetic valve who presents with recent dyspnoea or an embolic event. The diagnosis should be confirmed by TTE and TOE, cinefluoroscopy or CT scan if promptly available. ${ }^{169,170}$

The management of mechanical prosthetic valve thrombosis is high risk, whatever the option taken. Surgery is high risk because it is most often performed under emergency conditions and is a reintervention. On the other hand, fibrinolysis carries risks of bleeding, systemic embolism and recurrent thrombosis that are higher than after surgery. ${ }^{198}$

Emergency valve replacement is recommended for obstructive prosthetic valve thrombosis in critically ill patients without a contraindication to surgery (see table of recommendations in section 11.2.3 for management of prosthetic dysfunction and Figure 9).

Management of non-obstructive mechanical prosthetic valve thrombosis depends mainly on the occurrence of a thromboembolic event and the size of the thrombus (Figure 10). Surgery should be considered for a large $(>10 \mathrm{~mm})$ non-obstructive prosthetic valve thrombus complicated by embolism or which persists despite optimal anticoagulation. ${ }^{199}$ Fibrinolysis may be considered if surgery is at high risk but carries a risk of bleeding and thromboembolism.

Valve thrombosis occurs mainly in mechanical prostheses. However, cases of thrombosis of bioprostheses have been reported after surgery or transcatheter valve implantation. ${ }^{200,201}$ Subclinical thrombosis of bioprostheses may be more frequent when assessed by cardiac $\mathrm{CT},{ }^{202}$ and subclinical thrombosis of TAVI prostheses can be associated with a moderate increase in transprosthetic gradients, but the clinical consequences are unknown. ${ }^{203}$

Anticoagulation using a VKA and/or UFH is the first-line treatment of bioprosthetic valve thrombosis.

\section{Management of prosthetic valve dysfunction}

\begin{tabular}{|c|c|c|}
\hline Recommendations & Class $^{a}$ & Level $^{\mathbf{b}}$ \\
\hline \multicolumn{3}{|l|}{ Mechanical prosthetic thrombosis } \\
\hline $\begin{array}{l}\text { Urgent or emergency valve replacement is recom- } \\
\text { mended for obstructive thrombosis in critically ill } \\
\text { patients without serious comorbidity. }\end{array}$ & $\mathbf{I}$ & C \\
\hline $\begin{array}{l}\text { Fibrinolysis (using recombinant tissue plasminogen } \\
\text { activator } 10 \mathrm{mg} \text { bolus }+90 \mathrm{mg} \text { in } 90 \text { min with UFH or } \\
\text { streptokinase } 1500000 \mathrm{U} \text { in } 60 \mathrm{~min} \text { without UFH) } \\
\text { should be considered when surgery is not available or } \\
\text { is very high risk or for thrombosis of right-sided } \\
\text { prostheses. }\end{array}$ & Illa & C \\
\hline $\begin{array}{l}\text { Surgery should be considered for large ( }>10 \mathrm{~mm}) \\
\text { non-obstructive prosthetic thrombus complicated by } \\
\text { embolism. }\end{array}$ & Illa & C \\
\hline \multicolumn{3}{|l|}{ Bioprosthetic thrombosis } \\
\hline $\begin{array}{l}\text { Anticoagulation using a VKA and/or UFH is recom- } \\
\text { mended in bioprosthetic valve thrombosis before con- } \\
\text { sidering reintervention. }\end{array}$ & $\mathbf{I}$ & C \\
\hline \multicolumn{3}{|l|}{ Haemolysis and paravalvular leak } \\
\hline $\begin{array}{l}\text { Reoperation is recommended if paravalvular leak is } \\
\text { related to endocarditis or causes haemolysis requiring } \\
\text { repeated blood transfusions or leading to severe } \\
\text { symptoms. }\end{array}$ & $\mathbf{I}$ & C \\
\hline $\begin{array}{l}\text { Transcatheter closure may be considered for para- } \\
\text { valvular leaks with clinically significant regurgitation in } \\
\text { surgical high-risk patients (Heart Team decision). }\end{array}$ & Illb & C \\
\hline \multicolumn{3}{|l|}{ Bioprosthetic failure } \\
\hline $\begin{array}{l}\text { Reoperation is recommended in symptomatic patients } \\
\text { with a significant increase in transprosthetic gradient } \\
\text { (after exclusion of valve thrombosis) or severe } \\
\text { regurgitation. }\end{array}$ & $\mathbf{I}$ & C \\
\hline $\begin{array}{l}\text { Reoperation should be considered in asymptomatic } \\
\text { patients with significant prosthetic dysfunction if reop- } \\
\text { eration is at low risk. }\end{array}$ & IIa & C \\
\hline $\begin{array}{l}\text { Transcatheter valve-in-valve implantation in the aortic } \\
\text { position should be considered by the Heart Team } \\
\text { depending on the risk of reoperation and the type and } \\
\text { size of prosthesis. }\end{array}$ & Ila & C \\
\hline
\end{tabular}

$\mathrm{UFH}=$ unfractionated heparin; VKA = vitamin $\mathrm{K}$ antagonist.

${ }^{\mathrm{a}}$ Class of recommendation.

${ }^{\mathrm{b}}$ Level of evidence. 


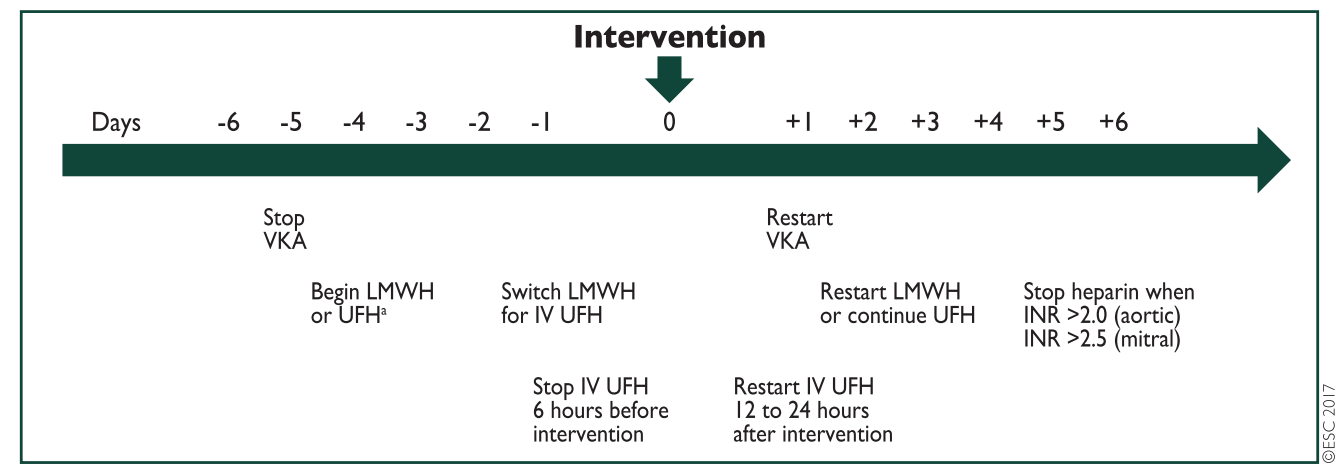

Figure 8 Main bridging steps for an intervention requiring interruption of oral anticoagulation in a patient with a mechanical prosthesis. Timing should be individualized according to patient characteristics, actual INR, and the type of intervention (reproduced with permission from lung and Rodes-Cabau ${ }^{42}$ ). INR = international normalized ratio; IV = intravenous; LMWH = low-molecular-weight heparin; UFH = unfractionated heparin; $\mathrm{VKA}=$ vitamin $\mathrm{K}$ antagonist.

aV UFH may be favoured in patients at high thrombotic risk.

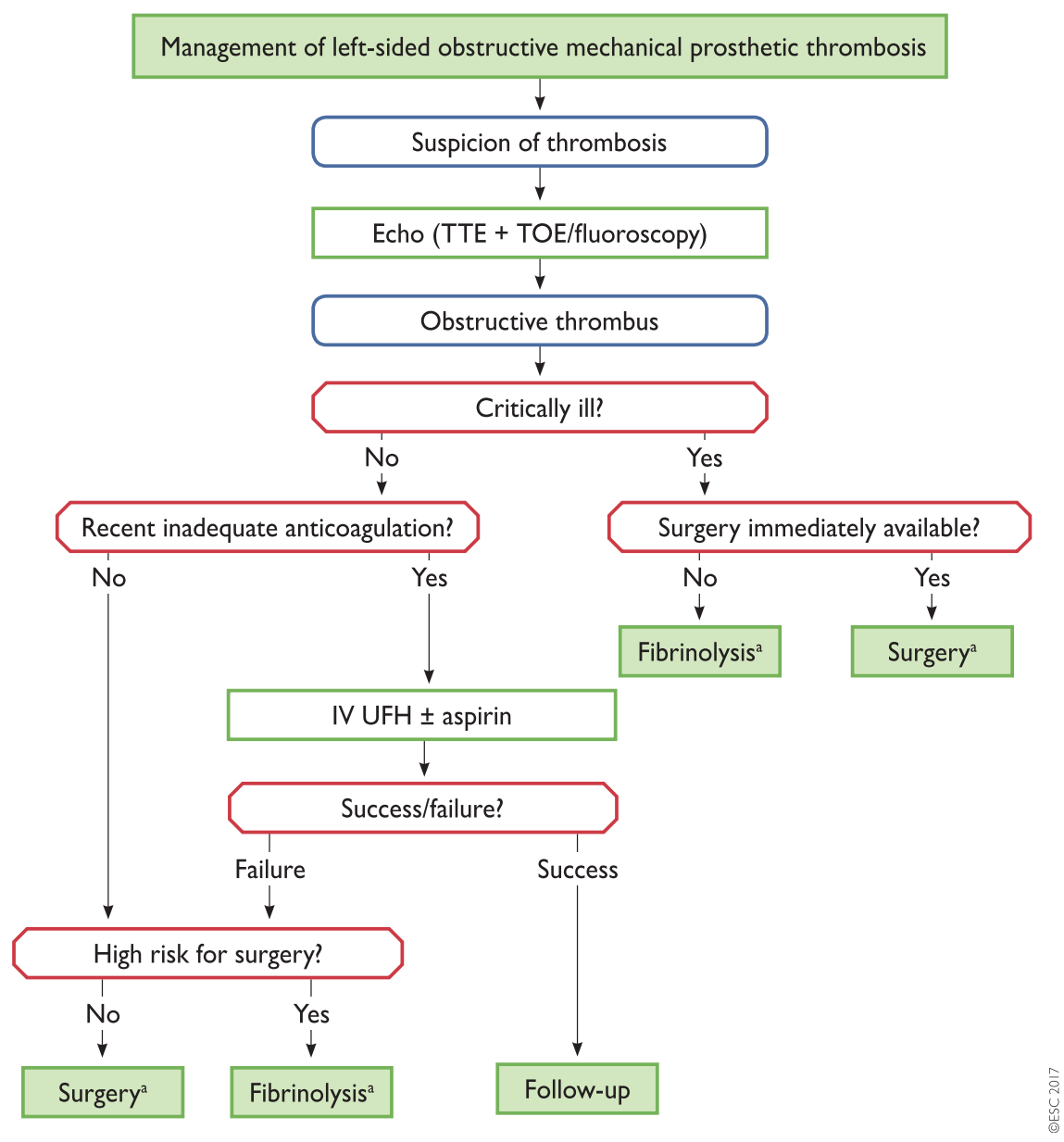

Figure 9 Management of left-sided obstructive mechanical prosthetic thrombosis. IV = intravenous; TOE = transoesophageal echocardiography; TTE = transthoracic echocardiography; UFH = unfractionated heparin.

${ }^{a}$ Risk and benefits of both treatments should be individualized. The presence of a first-generation prosthesis is an incentive to surgery. 


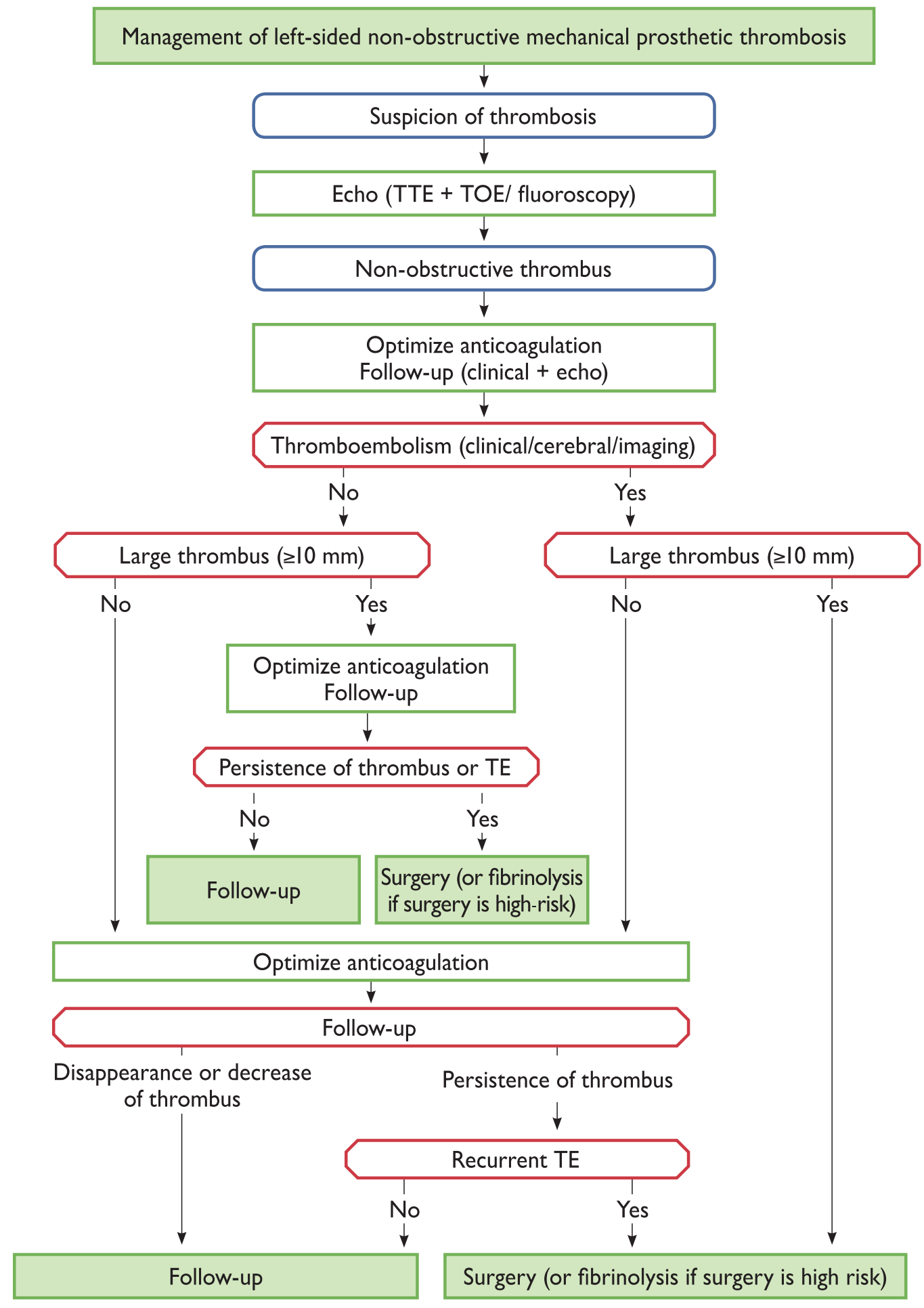

Figure IO Management of left-sided non-obstructive mechanical prosthetic thrombosis. TE = thromboembolism; TOE = transoesophageal echocardiography; TTE = transthoracic echocardiography. 


\subsubsection{Management of thromboembolism}

Thromboembolism after valve surgery is multifactorial in origin. ${ }^{171}$ Thorough investigation of each episode of thromboembolism is therefore essential (including cardiac and non-cardiac imaging) (Figure 10) rather than simply increasing the target INR or adding an antiplatelet agent. Prevention of further thromboembolic events involves the treatment of risk factors, optimization of anticoagulation control and the addition of low-dose aspirin ( $\leq 100 \mathrm{mg}$ daily) after careful analysis of the risk-benefit ratio.

\subsubsection{Management of haemolysis and paravalvular leak}

Blood tests for haemolysis should be part of routine follow-up after valve replacement. Lactate dehydrogenase, although non-specific, is related to the severity of haemolysis. The diagnosis of haemolytic anaemia requires TOE to detect a paravalvular leak if TTE is not contributory. Reoperation is recommended if the paravalvular leak is related to endocarditis or causes haemolysis requiring repeated blood transfusions or leading to severe symptoms (see table of recommendations in section 11.2.3 for management of prosthetic dysfunction). Medical therapy, including iron supplementation, betablockers and erythropoietin, is indicated in patients with severe haemolytic anaemia when contraindications to surgery are present. Transcatheter closure of a paravalvular leak is feasible, but experience is limited and there is presently no conclusive evidence to show a consistent efficiency. ${ }^{204}$

\subsubsection{Management of bioprosthetic valve failure}

After transcatheter as well as surgical implantation of a bioprosthetic valve, echocardiography including the measurement of transprosthetic gradients should be performed within 30 days (preferably 30 days for surgery) after valve implantation (i.e. baseline imaging), at 1 year after implantation and annually thereafter. ${ }^{168}$ The definitions of structural valve deterioration and bioprosthetic valve failure have recently been standardized in a consensus publication. ${ }^{168}$

Indications for reintervention are detailed in the table of recommendations for management of prosthetic dysfunction (section 11.2.3.).

Percutaneous balloon interventions should be avoided in the treatment of stenotic left-sided bioprostheses.

Transcatheter valve-in-valve implantation is now an option for treating degenerated bioprostheses in patients with increased surgical risk. Experience is mostly for bioprostheses in the aortic position and remains limited in the mitral position and even more so in the tricuspid position. ${ }^{205,206}$ Valve-in-valve and valve-in-ring procedures may be reasonable alternatives if the patient is at increased surgical risk, but it is necessary that the multidisciplinary Heart Team discusses every patient and chooses the best individualized approach.

\subsubsection{Heart failure}

Heart failure after valve surgery should lead to a search for prosthetic dysfunction or prosthesis-patient mismatch, deterioration of repair, LV dysfunction or progression of another valve disease. Non-valvular-related causes such as CAD, hypertension or sustained arrhythmias should also be considered. The management of patients with heart failure should follow the relevant guidelines. $^{113}$

\section{Key points}

- The choice between a mechanical prosthesis and a bioprosthesis should not overstress the role of age and should take into account the wishes of the informed patient.

- Patients with a mechanical prosthesis require lifelong treatment using VKA with a target INR adapted to the prosthesis and patient characteristics.

- Low-dose aspirin should be added to VKA only in selected patients with a mechanical prosthesis who have atherosclerosis or recurrent embolism.

- The risk of thromboembolism and bleeding is higher during the postoperative period and requires increased awareness of the monitoring of anticoagulant therapy.

- The management of anticoagulant therapy during non-cardiac surgery should be adapted to the type of surgery. Minor surgical procedures generally do not require interruption of anticoagulation.

Gaps of evidence

- The safety and efficacy of very-low-target INRs (median <2.5) in patients with a mechanical prosthesis in the aortic position should be further studied.

- The safety and efficacy of NOACs in patients with a mechanical prosthesis require further research.

- The safety and efficacy of low-dose aspirin associated with contemporary target INRs in patients with a mechanical prosthesis, according to the presence or absence of atherosclerosis, require further evaluation.

- Optimal early antithrombotic therapy after implantation of surgical and transcatheter aortic bioprostheses needs to be better defined.

- Long-term outcome data of transcatheter valve-in-valve and valve-in-ring procedures are required.

\section{Management during non- cardiac surgery}

Cardiovascular morbidity and mortality are increased in patients with VHD who undergo non-cardiac surgery. Symptomatic severe aortic stenosis or mitral stenosis may require valve replacement or percutaneous intervention before non-cardiac surgery. A detailed description of these recommendations is available. ${ }^{196}$

\subsection{Preoperative evaluation}

Echocardiography should be performed in any patient with VHD. Determination of functional capacity is a pivotal step in preoperative risk assessment, measured either by exercise test or ability to perform activities in daily life. The decision for management should be 


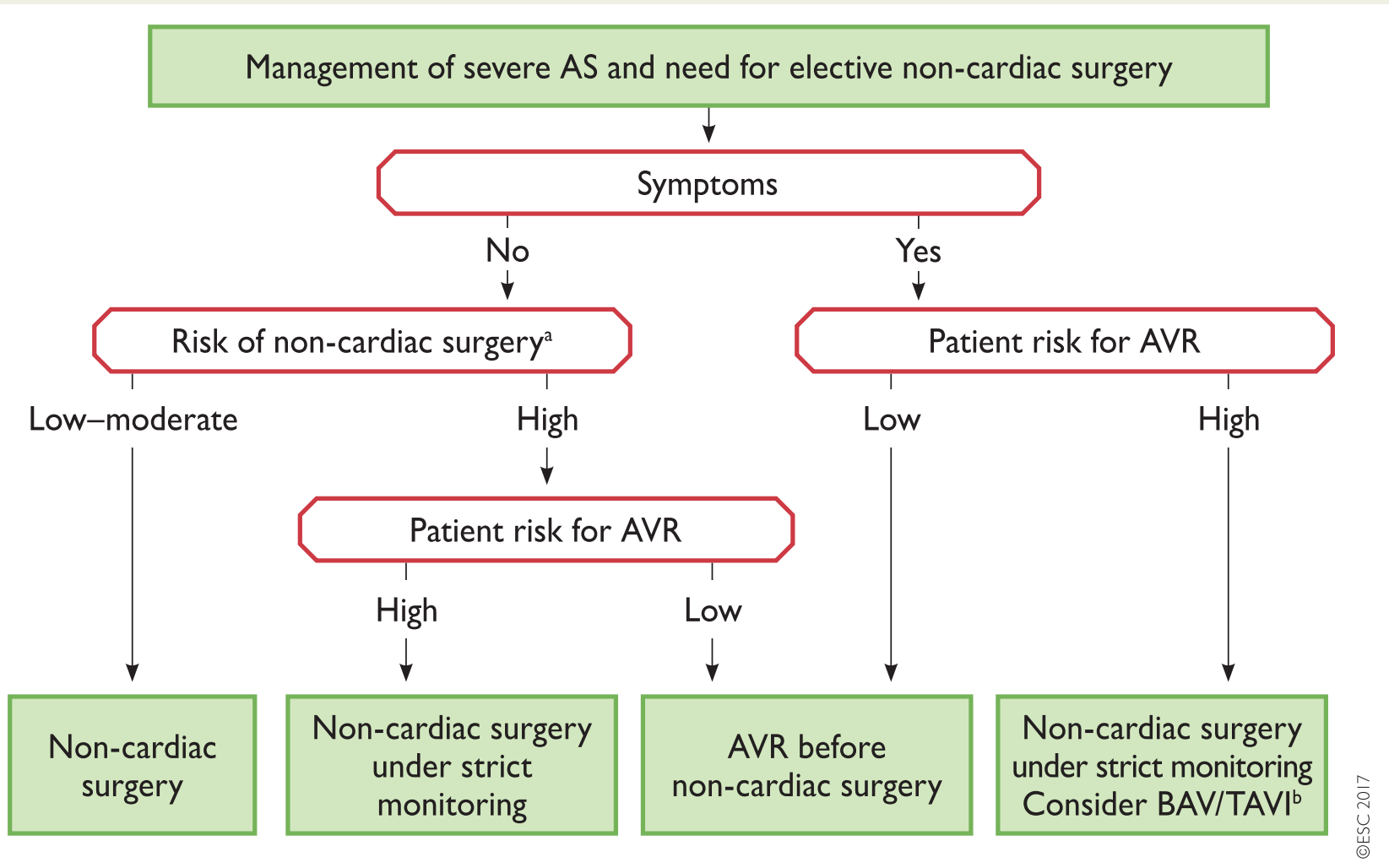

Figure I I Management of severe aortic stenosis and elective non-cardiac surgery according to patient characteristics and type of surgery. AS = aortic stenosis; $\mathrm{AVR}=$ aortic valve replacement; $\mathrm{BAV}=$ balloon aortic valvuloplasty; $\mathrm{TAVI}=$ transcatheter aortic valve implantation.

${ }^{a}$ Classification into three groups according to the risk of cardiac complications (30-day death and myocardial infarction) for non-cardiac surgery (high-risk $>5 \%$; intermediate risk $1-5 \%$; low risk $<1 \%$ ). ${ }^{196}$

${ }^{b}$ Non-cardiac surgery performed only if strictly needed. The choice between percutaneous aortic valvuloplasty and TAVI should take into account patient life expectancy.

taken after multidisciplinary discussion involving cardiologists, surgeons and anaesthesiologists.

\subsection{Specific valve lesions}

\subsubsection{Aortic stenosis}

In patients with severe aortic stenosis, urgent non-cardiac surgery should be performed under careful haemodynamic monitoring.

The management related to elective non-cardiac surgery depends on the presence of symptoms and the type of surgery. ${ }^{196,207,208}$ In symptomatic patients, aortic valve replacement should be considered before non-cardiac surgery. In patients at increased surgical risk, TAVI is a therapeutic option. In asymptomatic patients, elective non-cardiac surgery can be performed safely, albeit with a risk of worsening heart failure. ${ }^{207,208}$ If noncardiac surgery implies large volume shifts, aortic valve replacement should be considered first (Figure 11).

\subsubsection{Mitral stenosis}

Non-cardiac surgery can be performed safely in patients with nonsignificant mitral stenosis (valve area $>1.5 \mathrm{~cm}^{2}$ ) and in asymptomatic patients with significant mitral stenosis and a systolic pulmonary artery pressure $<50 \mathrm{mmHg}$.

In symptomatic patients or in patients with systolic pulmonary artery pressure $>50 \mathrm{mmHg}$, correction of mitral stenosis, by means of PMC whenever possible, should be attempted before non-cardiac surgery if it is high risk.

\subsubsection{Aortic and mitral regurgitation}

Non-cardiac surgery can be performed safely in asymptomatic patients with severe mitral regurgitation or aortic regurgitation and preserved LV function. The presence of symptoms or LV dysfunction should lead to consideration of valvular surgery, but this is seldom needed before non-cardiac surgery. If LV dysfunction is severe (ejection fraction $<30 \%$ ), non-cardiac surgery should be performed only if strictly necessary, after optimization of medical therapy for heart failure. 


\subsection{Perioperative monitoring}

Heart rate control (particularly in mitral stenosis) and careful fluid management (particularly in aortic stenosis) are needed. TOE monitoring may be considered.

\section{Key points}

- In symptomatic patients with severe aortic stenosis, aortic valve replacement or TAVI should be considered before non-cardiac surgery.

- In patients with severe mitral stenosis and symptoms or pulmonary artery pressure $>50 \mathrm{mmHg}$, PMC should be attempted before non-cardiac surgery.

\section{Management during pregnancy}

Detailed guidelines on the management of cardiovascular disease during pregnancy are available in another document. ${ }^{209}$

The decision for management during pregnancy should be taken after multidisciplinary discussion involving cardiologists, obstetricians and anaesthesiologists. ${ }^{209}$ Valve disease should be evaluated before pregnancy and treated if necessary. Pregnancy should be discouraged in severe mitral stenosis, severe symptomatic aortic stenosis and with an aortic diameter $>45 \mathrm{~mm}$ in Marfan syndrome or $>27.5 \mathrm{~mm} / \mathrm{m}^{2}$ in Turner syndrome.

Caesarean section is recommended for patients with severe mitral or aortic stenosis, ascending aortic diameter $>45 \mathrm{~mm}$ or severe pulmonary hypertension, as well as women on oral anticoagulants in preterm labour.

\subsection{Native valve disease}

Moderate or severe mitral stenosis with a valve area $<1.5 \mathrm{~cm}^{2}$ in pregnant women is usually poorly tolerated. PMC should be considered in severely symptomatic patients (NYHA class III-IV) and/or those with systolic pulmonary artery pressure $>50 \mathrm{mmHg}$ despite optimal therapy. PMC should be performed after the 20th week of pregnancy in experienced centres. ${ }^{209}$

Complications of severe aortic stenosis occur mainly in patients who were symptomatic before pregnancy and among those with impaired LV function. Evaluation with an exercise test is recommended before pregnancy.

Chronic mitral regurgitation and aortic regurgitation are well tolerated, even when severe, provided LV systolic function is preserved.

Surgery under cardiopulmonary bypass is associated with a foetal mortality rate of $15-30 \%{ }^{210}$ and should be restricted to the rare conditions that threaten the mother's life.

\subsection{Prosthetic valves}

Maternal mortality is estimated at $1-4 \%$ and serious events occur in up to $40 \%$ of women with mechanical valves. ${ }^{211}$

Therapeutic anticoagulation is extremely important to avoid complications. In patients requiring $\leq 5 \mathrm{mg}$ warfarin, oral anticoagulants throughout pregnancy and a change to UFH before delivery is favoured. In patients requiring higher doses, switching to LMWH during the first trimester with strict anti-Xa monitoring (therapeutic range $0.8-1.2 \mathrm{IU} / \mathrm{mL}$ ) and the use of oral anticoagulants afterwards is favoured. $^{209}$

\section{Key points}

- Pregnancy should be discouraged in women with severe mitral stenosis and severe symptomatic aortic stenosis.

- Pregnancy in women with a mechanical valve, especially in the mitral position, is associated with a high risk for maternal and foetal complications. Therapeutic anticoagulation during pregnancy is of utmost importance in these patients.

\section{Gaps in evidence}

The optimal management of pregnant women with mechanical heart valves with regards to the antithrombotic regimen needs to be better defined. 


\section{To do and not to do messages from the Guidelines}

\begin{tabular}{|c|c|c|}
\hline Recommendations & Class $^{\mathrm{a}}$ & Level $^{\mathrm{b}}$ \\
\hline \multicolumn{3}{|l|}{ Management of CAD in patients with VHD (adapted from Windecker et al. ${ }^{16}$ ) } \\
\hline $\begin{array}{l}\text { Coronary angiographyc is recommended before valve surgery in patients with severe VHD and any of the } \\
\text { following: } \\
\text { - history of cardiovascular disease } \\
\text { - suspected myocardial ischaemiad } \\
\text { - LV systolic dysfunction } \\
\text { - in men aged over } 40 \text { years and postmenopausal women } \\
\text { - one or more cardiovascular risk factors. }\end{array}$ & 1 & C \\
\hline $\begin{array}{l}\text { Coronary angiography is recommended in the evaluation of moderate to severe secondary mitral } \\
\text { regurgitation. }\end{array}$ & 1 & c \\
\hline $\begin{array}{l}\text { CABG is recommended in patients with a primary indication for aortic/mitral valve surgery and coronary artery } \\
\text { diameter stenosis } \geq 70 \% \text {. }\end{array}$ & I & C \\
\hline \multicolumn{3}{|l|}{ Management of atrial fibrillation in patients with VHD } \\
\hline $\begin{array}{l}\text { The use of NOACs is not recommended in patients with atrial fibrillation and moderate to severe mitral } \\
\text { stenosis. }\end{array}$ & III & C \\
\hline NOACS are contraindicated in patients with a mechanical valve. ${ }^{45}$ & III & B \\
\hline \multicolumn{3}{|l|}{ Indications for surgery } \\
\hline \multicolumn{3}{|l|}{ (A) Severe aortic regurgitation } \\
\hline Surgery is indicated in symptomatic patients. ${ }^{57,58,66,67}$ & 1 & B \\
\hline Surgery is indicated in asymptomatic patients with resting LVEF $\leq 50 \% .57,58$ & $\mathbf{I}$ & B \\
\hline Surgery is indicated in patients undergoing CABG or surgery of the ascending aorta, or of another valve. & 1 & C \\
\hline $\begin{array}{l}\text { Heart Team discussion is recommended in selected patientsc in whom aortic valve repair may be a feasible } \\
\text { alternative to valve replacement. }\end{array}$ & 1 & C \\
\hline \multicolumn{3}{|l|}{ (B) Aortic root disease (irrespective of the severity of aortic regurgitation) } \\
\hline $\begin{array}{l}\text { Aortic valve repair, using the reimplantation or remodelling with aortic annuloplasty technique, is } \\
\text { recommended in young patients with aortic root dilation and tricuspid aortic valves, when performed by } \\
\text { experienced surgeons. }\end{array}$ & I & C \\
\hline $\begin{array}{l}\text { Surgery is indicated in patients with Marfan syndrome, who have aortic root disease with a maximal ascending } \\
\text { aortic diameter } \geq 50 \mathrm{~mm} \text {. }\end{array}$ & 1 & C \\
\hline \multicolumn{3}{|l|}{ Indications for intervention in aortic stenosis and recommendations for the choice of intervention mode } \\
\hline $\begin{array}{l}\text { Intervention is indicated in symptomatic patients with severe, high-gradient aortic stenosis (mean gradient } \\
\geq 40 \mathrm{mmHg} \text { or peak velocity } \geq 4.0 \mathrm{~m} / \mathrm{s} \text { ). }{ }^{91-93}\end{array}$ & 1 & B \\
\hline $\begin{array}{l}\text { Intervention is indicated in symptomatic patients with severe low-flow, low-gradient ( }<40 \mathrm{mmHg} \text { ) aortic } \\
\text { stenosis with reduced ejection fraction, and evidence of flow (contractile) reserve excluding pseudosevere } \\
\text { aortic stenosis. }\end{array}$ & I & C \\
\hline $\begin{array}{l}\text { Intervention should not be performed in patients with severe comorbidities when the intervention is unlikely } \\
\text { to improve quality of life or survival. }\end{array}$ & III & C \\
\hline $\begin{array}{l}\text { Aortic valve interventions should only be performed in centres with both departments of cardiology and } \\
\text { cardiac surgery on-site, and with structured collaboration between the two, including a Heart Team (heart } \\
\text { valve centres). }\end{array}$ & 1 & C \\
\hline $\begin{array}{l}\text { The choice for intervention must be based on careful individual evaluation of technical suitability and } \\
\text { weighing of risks and benefits of each modality (aspects to be considered are listed in Table 7). In addition, } \\
\text { the local expertise and outcomes data for the given intervention must be taken into account. }\end{array}$ & 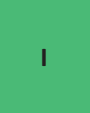 & C \\
\hline $\begin{array}{l}\text { SAVR is recommended in patients at low surgical risk (STS or EuroSCORE II }<4 \% \text { or logistic EuroSCORE I }<10 \% \\
\text { and no other risk factors not included in these scores, such as frailty, porcelain aorta, sequelae of } \\
\text { chest radiation). }\end{array}$ & 1 & B \\
\hline TAVI is recommended in patients who are not suitable for SAVR as assessed by the Heart Team. ${ }^{91,94}$ & I & B \\
\hline $\begin{array}{l}\text { In patients who are at increased surgical risk (STS or EuroSCORE II } \geq 4 \% \text { or logistic EuroSCORE I } \geq 10 \% \text {, or other } \\
\text { risk factors not included in these scores such as frailty, porcelain aorta, sequelae of chest radiation), } \\
\text { the decision between SAVR and TAVI should be made by the Heart Team according to the individual } \\
\text { patient characteristics (see Table 7), with TAVI being favoured in elderly patients suitable for } \\
\text { transfemoral access. }{ }^{91,94-102}\end{array}$ & I & B \\
\hline $\begin{array}{l}\text { SAVR is indicated in asymptomatic patients with severe aortic stenosis and systolic LV dysfunction (LVEF }<50 \% \text { ) } \\
\text { not due to another cause. }\end{array}$ & I & C \\
\hline $\begin{array}{l}\text { SAVR is indicated in asymptomatic patients with severe aortic stenosis and abnormal exercise test showing } \\
\text { symptoms on exercise clearly related to aortic stenosis. }\end{array}$ & $\mathbf{I}$ & C \\
\hline $\begin{array}{l}\text { SAVR is indicated in patients with severe aortic stenosis undergoing CABG, or surgery of the ascending aorta } \\
\text { or of another valve. }\end{array}$ & I & c \\
\hline
\end{tabular}


Indications for intervention in severe primary mitral regurgitation

Mitral valve repair should be the preferred technique when the results are expected to be durable.

Surgery is indicated in symptomatic patients with LVEF $>30 \%$. $^{121,131,132}$

Surgery is indicated in asymptomatic patients with LV dysfunction (LVESD $\geq 45 \mathrm{~mm}$ and/or LVEF $\leq 60 \%)^{122,131}$

\begin{tabular}{|c|c|}
\hline I & C \\
\hline I & B \\
\hline I & B \\
\hline
\end{tabular}

Indications for mitral valve intervention in chronic secondary mitral regurgitation

Surgery is indicated in patients with severe secondary mitral regurgitation undergoing CABG and LVEF $>30 \%$.

Indications for PMC and mitral valve surgery in clinically significant (moderate or severe) mitral stenosis (valve area $\leq 1.5 \mathrm{~cm}^{2}$ )

PMC is indicated in symptomatic patients without unfavourable characteristics for PMC. ${ }^{144,146,148}$

PMC is indicated in any symptomatic patients with a contraindication or a high risk for surgery.

Mitral valve surgery is indicated in symptomatic patients who are not suitable for PMC.

Indications for tricuspid valve surgery

Surgery is indicated in symptomatic patients with severe tricuspid stenosis.

Surgery is indicated in patients with severe tricuspid stenosis undergoing left-sided valve intervention.

Surgery is indicated in patients with severe primary tricuspid regurgitation undergoing left-sided valve surgery.

Surgery is indicated in symptomatic patients with severe isolated primary tricuspid regurgitation without severe right-ventricular dysfunction.

Surgery is indicated in patients with severe secondary tricuspid regurgitation undergoing left-sided valve surgery.

Choice of the aortic/mitral prosthesis - in favour of a mechanical prosthesis; the decision is based on the integration of several of the following factors

A mechanical prosthesis is recommended according to the desire of the informed patient and if there are no contraindications to long-term anticoagulation.

A mechanical prosthesis is recommended in patients at risk of accelerated structural valve deterioration.

\begin{tabular}{l|l|l|l}
\hline & C
\end{tabular}

\begin{tabular}{|l|l|l|}
\hline I & B \\
\hline I & C \\
\hline I & C \\
\hline
\end{tabular}

Choice of the aortic/mitral prosthesis - in favour of a bioprosthesis; the decision is based on the integration of several of the following factors

A bioprosthesis is recommended according to the desire of the informed patient.

A bioprosthesis is recommended when good-quality anticoagulation is unlikely (compliance problems, not readily available) or contraindicated because of high bleeding risk (previous major bleed, comorbidities, unwillingness, compliance problems, lifestyle, occupation).

A bioprosthesis is recommended for reoperation for mechanical valve thrombosis despite good long-term anticoagulant control.

Indications for antithrombotic therapy in patients with mechanical prostheses and bioprostheses

Mechanical prostheses

Oral anticoagulation using a VKA is recommended lifelong for all patients. ${ }^{179,180}$

Bridging using therapeutic doses of UFH or LMWH is recommended when VKA treatment should be interrupted.

INR self-management is recommended provided appropriate training and quality control are performed. ${ }^{181}$

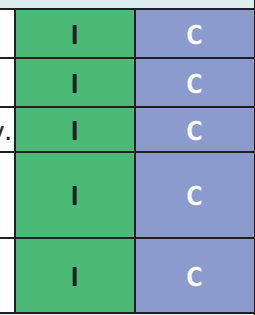

The use of NOACs is contraindicated. ${ }^{45}$

Bioprostheses

Oral anticoagulation is recommended lifelong for patients with surgical or transcatheter implanted bioprostheses who have other indications for anticoagulation.

Management of prosthetic valve dysfunction

Urgent or emergency valve replacement is recommended for obstructive thrombosis in critically ill patients without serious comorbidity.

Anticoagulation using a VKA and/or UFH is recommended in bioprosthetic valve thrombosis before considering reintervention.

Reoperation is recommended if paravalvular leak is related to endocarditis or causes haemolysis requiring repeated blood transfusions or leading to severe symptoms.

Reoperation is recommended in symptomatic patients with a significant increase in transprosthetic gradient (after exclusion of valve thrombosis) or severe regurgitation.

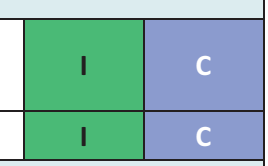




\section{What is new in the 2017 Valvular Heart Disease Guidelines?}

What is new in the 2017 Valvular Heart Disease Guidelines?

\begin{tabular}{|c|c|}
\hline \multicolumn{2}{|c|}{ Changes in recommendations } \\
\hline 2012 & 2017 \\
\hline \multicolumn{2}{|l|}{ Indications for intervention in symptomatic aortic stenosis } \\
\hline $\begin{array}{l}\text { IIb C } \\
\text { Intervention may be considered in symptomatic patients with } \\
\text { low-flow, low-gradient aortic stenosis and reduced ejection } \\
\text { fraction without flow (contractile) reserve. }\end{array}$ & $\begin{array}{l}\text { Ila C } \\
\text { Intervention should be considered in symptomatic patients with low-flow, low- } \\
\text { gradient aortic stenosis and reduced ejection fraction without flow (contractile) } \\
\text { reserve, particularly when CT calcium scoring confirms severe aortic stenosis. }\end{array}$ \\
\hline \multicolumn{2}{|l|}{ Choice of intervention in symptomatic aortic stenosis } \\
\hline $\begin{array}{l}\text { Recommendations for the use of TAVI } \\
\text { (Tables on "Contra-indications for TAVI" and Table on } \\
\text { "Recommendations for the use of TAVI") }\end{array}$ & $\begin{array}{l}\text { Replaced by recommendations for the choice of intervention } \\
\text { See Section b in Table "Indications for intervention in aortic stenosis and } \\
\text { recommendations for the choice of intervention" (Section 5.2), and Table } 7 \\
\text { "Aspects to be considered by the heart team for the decision between SAVR and } \\
\text { TAVI in patients at increased surgical risk" }\end{array}$ \\
\hline \multicolumn{2}{|l|}{ Indications for surgery in asymptomatic aortic stenosis } \\
\hline $\begin{array}{l}\text { IIb C } \\
\text { Markedly elevated BNP levels. }\end{array}$ & $\begin{array}{l}\text { Ila C } \\
\text { Markedly elevated BNP levels (>threefold age- and sex-corrected normal range) } \\
\text { confirmed by repeated measurements without other explanations. }\end{array}$ \\
\hline $\begin{array}{l}\text { IIlb C } \\
\text { Increase of mean pressure gradient with exercise by }>20 \\
\mathrm{mmHg} \text {. }\end{array}$ & Taken out \\
\hline $\begin{array}{l}\text { IIb C } \\
\text { Excessive LV hypertrophy in the absence of hypertension. }\end{array}$ & Taken out \\
\hline \multicolumn{2}{|c|}{ Indications for intervention in asymptomatic severe primary mitral regurgitation } \\
\hline $\begin{array}{l}\text { IIb C } \\
\text { Surgery may be considered in asymptomatic patients with } \\
\text { preserved LV function, high likelihood of durable repair, low } \\
\text { surgical risk, and: } \\
\text { - Left atrial dilatation (volume index } \geq 60 \mathrm{~mL} / \mathrm{m}^{2} \mathrm{BSA} \text { ) and } \\
\text { sinus rhythm }\end{array}$ & $\begin{array}{l}\text { IIa C (modified!) } \\
\text { Surgery should be considered in asymptomatic patients with preserved LVEF } \\
(>60 \% \text { ) and LVESD } 40-44 \mathrm{~mm} \text { when a durable repair is likely, surgical risk is low, } \\
\text { the repair is performed in heart valve centres, and the following finding is present: } \\
\text { presence of significant LA dilatation (volume index } 260 \mathrm{~mL} / \mathrm{m}^{2} \mathrm{BSA} \text { ) in sinus } \\
\text { rhythm. }\end{array}$ \\
\hline $\begin{array}{l}\text { Pulmonary hypertension on exercise (SPAP } \geq 60 \mathrm{mmHg} \text { at } \\
\text { exercise) }\end{array}$ & Taken out \\
\hline \multicolumn{2}{|c|}{ Indications for mitral valve intervention in secondary mitral regurgitation } \\
\hline $\begin{array}{l}\text { Illa C } \\
\text { Surgery should be considered in patients with moderate } \\
\text { secondary mitral regurgitation undergoing CABG }\end{array}$ & raken out \\
\hline \multirow[t]{4}{*}{$\begin{array}{l}\text { IIlb C } \\
\text { When revascularization is not indicated, surgery may } \\
\text { be considered in patients with severe secondary mitral } \\
\text { regurgitation and LVEF }>30 \% \text {, } \\
\text { who remain symptomatic despite optimal medical management } \\
\text { (including CRT if indicated). }\end{array}$} & $\begin{array}{l}\text { Illb C (modified) } \\
\text { When revascularization is not indicated, surgery may be considered in patients with } \\
\text { severe secondary mitral regurgitation and LVEF }>30 \% \text {, who remain symptomatic } \\
\text { despite optimal medical management (including CRT if indicated) and have a low } \\
\text { surgical risk. }\end{array}$ \\
\hline & $\begin{array}{l}\text { When revascularization is not indicated and surgical risk is not low, a percutaneous } \\
\text { edge-to-edge procedure may be considered in patients with severe secondary mitral } \\
\text { regurgitation and LVEF }>30 \% \text {, who remain symptomatic despite optimal medical } \\
\text { management (including CRT if indicated) and who have a suitable valve morphology by } \\
\text { echocardiography, avoiding futility. }\end{array}$ \\
\hline & $\begin{array}{l}\text { In patients with severe secondary mitral regurgitation and LVEF }<30 \% \text { who remain } \\
\text { symptomatic despite optimal medical management (including CRT if indicated) and } \\
\text { who have no option for revascularization, the Heart Team may consider percutaneous } \\
\text { edge-to-edge procedure or valve surgery after careful evaluation for ventricular assist } \\
\text { device or heart transplant according to individual patient characteristics. }\end{array}$ \\
\hline & $\begin{array}{l}\text { Additional statement: } \\
\text { The lower thresholds defining severe MR compared to primary MR are based on their } \\
\text { association with prognosis. However, it is unclear if prognosis is independently affected } \\
\text { by MR compared to LV dysfunction. For isolated mitral valve treatment in secondary } \\
\text { MR, thresholds of severity of MR for intervention still need to be validated in clinical } \\
\text { trials. So far, no survival benefit has been confirmed for reduction of secondary MR. }\end{array}$ \\
\hline \multicolumn{2}{|c|}{ Indications for antithrombotic therapy in patients with a prosthetic heart valve or valve repair } \\
\hline & \\
\hline $\begin{array}{l}\text { The addition of low-dose aspirin }(75-100 \mathrm{mg} / \mathrm{day}) \text { to VKA should } \\
\text { be considered in the case of concomitant atherosclerotic disease. }\end{array}$ & $\begin{array}{l}\text { The addition of low-dose aspirin }(75-100 \mathrm{mg} / \text { day }) \text { to VKA may be considered in } \\
\text { the case of concomitant atherosclerotic disease. }\end{array}$ \\
\hline
\end{tabular}


What is new in the 2017 Valvular Heart Disease Guidelines? (continued)

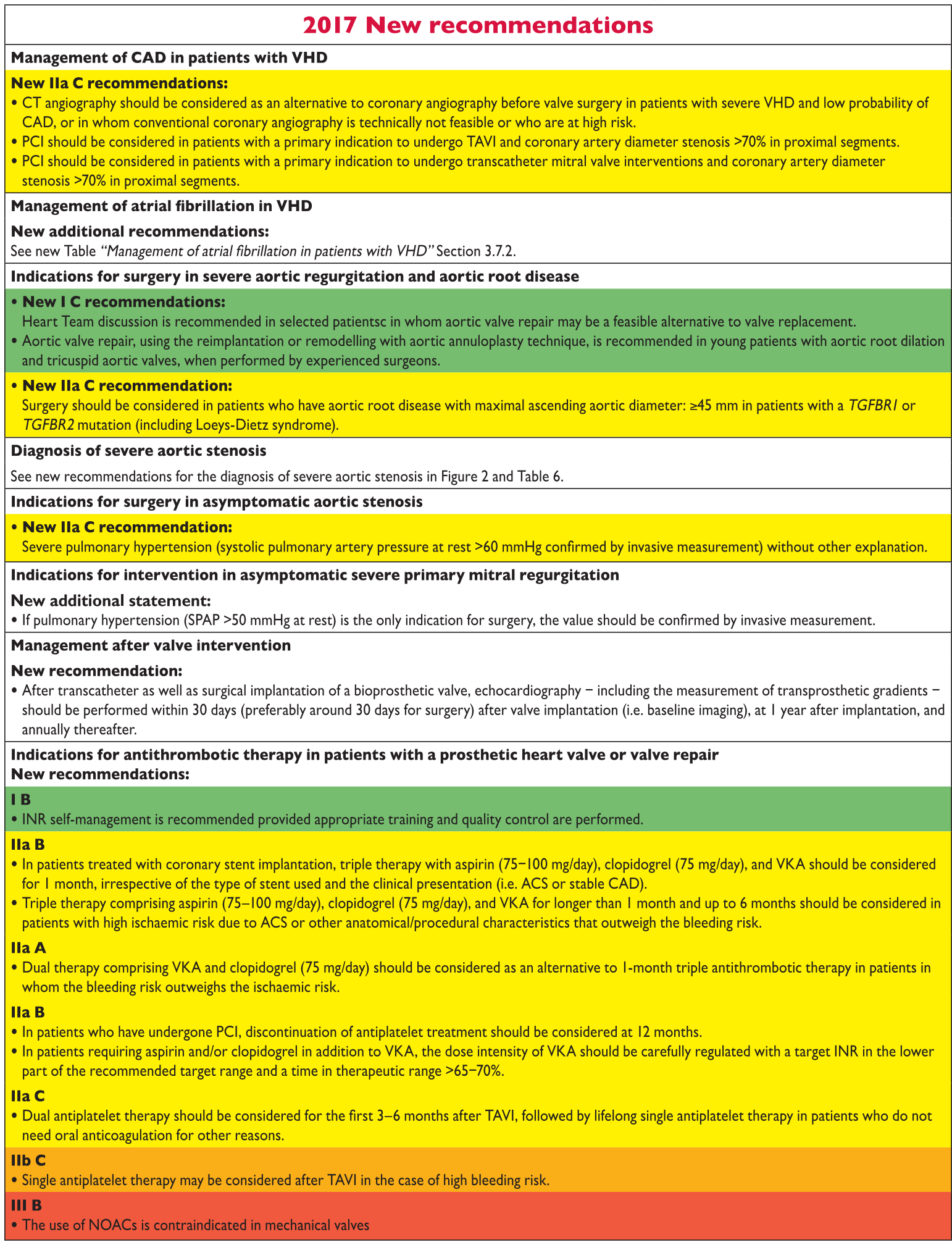


What is new in the 2017 Valvular Heart Disease Guidelines? (continued)

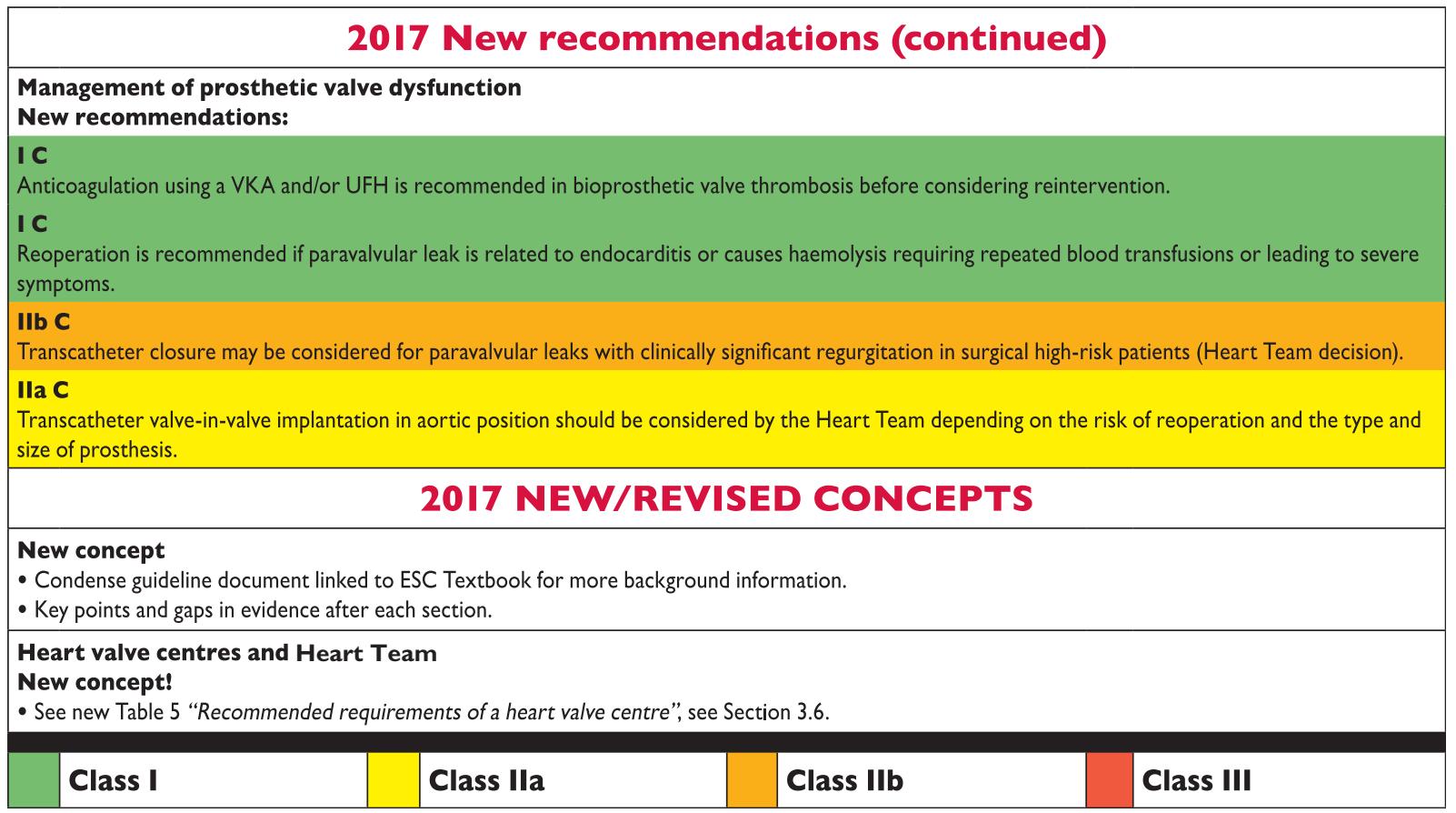

\section{Appendix}

ESC Committee for Practice Guidelines (CPG): Stephan Windecker (Chairperson) (Switzerland), Victor Aboyans (France), Stefan Agewall (Norway), Emanuele Barbato (Italy), Héctor Bueno (Spain), Antonio Coca (Spain), Jean-Philippe Collet (France), loan Mircea Coman (Romania), Veronica Dean (France), Victoria Delgado (The Netherlands), Donna Fitzsimons (UK), Oliver Gaemperli (Switzerland), Gerhard Hindricks (Germany), Bernard lung (France), Peter Jüni (Canada), Hugo A. Katus (Germany), Juhani Knuuti (Finland), Patrizio Lancellotti (Belgium), Christophe Leclercq (France), Theresa McDonagh (UK), Massimo Francesco Piepoli (Italy), Piotr Ponikowski (Poland), Dimitrios J. Richter (Greece), Marco Roffi (Switzerland), Evgeny Shlyakhto (Russia), lain A. Simpson (UK), Jose Luis Zamorano (Spain).

ESC National Cardiac Societies actively involved in the review process of the 2017 ESC/EACTS Guidelines for the management of valvular heart disease:

Armenia: Armenian Cardiologists Association, Hovhannes K. Kzhdryan; Austria: Austrian Society of Cardiology, Julia Mascherbauer; Azerbaijan: Azerbaijan Society of Cardiology, Fuad Samadov; Belarus: Belorussian Scientific Society of Cardiologists, Vadim Shumavets; Belgium: Belgian Society of Cardiology, Guy Van Camp; Bosnia and Herzegovina: Association of Cardiologists of Bosnia and Herzegovina, Daniela Lončar; Croatia: Croatian Cardiac Society, Daniel Lovric; Cyprus: Cyprus Society of Cardiology, Georgios M. Georgiou; Czech Republic: Czech Society of Cardiology,
Katerina Linhartova; Denmark: Danish Society of Cardiology, Nikolaj Ihlemann; Egypt: Egyptian Society of Cardiology, Magdy Abdelhamid; Estonia: Estonian Society of Cardiology, Teele Pern; Finland: Finnish Cardiac Society, Anu Turpeinen; Former Yugoslav Republic of Macedonia: Macedonian FYR Society of Cardiology, Elizabeta Srbinovska-Kostovska; France: French Society of Cardiology, Ariel Cohen; Georgia: Georgian Society of Cardiology, Zviad Bakhutashvili; Germany: German Cardiac Society, Hüseyin Ince; Greece: Hellenic Society of Cardiology, Manolis Vavuranakis; Hungary: Hungarian Society of Cardiology, András Temesvári; Iceland: Icelandic Society of Cardiology, Thorarinn Gudnason; Ireland: Irish Cardiac Society, Darren Mylotte; Israel: Israel Heart Society, Rafael Kuperstein; Italy: Italian Federation of Cardiology, Ciro Indolfi; Kazakhstan: Association of Cardiologists of Kazakhstan, Yury Pya; Kosovo: Kosovo Society of Cardiology, Gani Bajraktari; Kyrgyzstan: Kyrgyz Society of Cardiology, Alina Kerimkulova; Latvia: Latvian Society of Cardiology, Ainars Rudzitis; Lithuania: Lithuanian Society of Cardiology, Vaida Mizariene; Luxembourg: Luxembourg Society of Cardiology, Frédéric Lebrun; Malta: Maltese Cardiac Society, Daniela Cassar Demarco; Morocco: Moroccan Society of Cardiology, Latifa Oukerraj; The Netherlands: Netherlands Society of Cardiology, Berto J. Bouma; Norway: Norwegian Society of Cardiology, Terje Kristian Steigen; Poland: Polish Cardiac Society, Monika Komar; Portugal: Portuguese Society of Cardiology, Luisa Maria De Moura Branco; Romania: Romanian Society of Cardiology, Bogdan A. Popescu; Russian Federation: Russian Society of Cardiology, 
Vladimir Uspenskiy; San Marino: San Marino Society of Cardiology, Marina Foscoli; Serbia: Cardiology Society of Serbia, Ljiljana Jovovic; Slovakia: Slovak Society of Cardiology, Iveta Simkova; Slovenia: Slovenian Society of Cardiology, Matjaz Bunc; Spain: Spanish Society of Cardiology, José Antonio Vázquez de Prada; Sweden: Swedish Society of Cardiology, Martin Stagmo; Switzerland: Swiss Society of Cardiology, Beat Andreas Kaufmann; Tunisia: Tunisian Society of Cardiology and Cardio-Vascular Surgery, Abdallah Mahdhaoui; Turkey: Turkish Society of Cardiology, Engin Bozkurt; Ukraine: Ukrainian Association of Cardiology, Elena Nesukay; United Kingdom: British Cardiovascular Society, Stephen J. D. Brecker.

We wish to thank Professor A. Pieter Kappetein for his advice and contribution to these guidelines.

\section{References}

1. Popescu BA, Andrade MJ, Badano LP, Fox KF, Flachskampf FA, Lancellotti P, Varga A, Sicari R, Evangelista A, Nihoyannopoulos P, Zamorano JL, Derumeaux G, Kasprzak JD, Roelandt JR. European Association of Echocardiography recommendations for training, competence, and quality improvement in echocardiography. Eur J Echocardiogr 2009;10:893-905.

2. Lancellotti P, Tribouilloy C, Hagendorff A, Popescu BA, Edvardsen T, Pierard LA, Badano L, Zamorano JL, Scientific Document Committee of the European Association of Cardiovascular Imaging. Recommendations for the echocardiographic assessment of native valvular regurgitation: an executive summary from the European Association of Cardiovascular Imaging. Eur Heart J Cardiovasc Imaging 2013;14:611-644.

3. Baumgartner H, Hung J, Bermejo J, Chambers JB, Evangelista A, Griffin BP, lung B, Otto CM, Pellikka PA, Quinones M, EAE/ASE. Echocardiographic assessment of valve stenosis: EAE/ASE recommendations for clinical practice. Eur J Echocardiogr 2009;10:1-25.

4. Baumgartner $\mathrm{H}$, Hung J, Bermejo J, Chambers JB, Edvardsen T, Goldstein S, Lancellotti P, LeFevre M, Miller F, Otto CM. Focus update on the echocardiographic assessment of aortic valve stenosis: EAE/ASE recommendations for clinical practice. Eur Heart J Cardiovasc Imaging 2017;18:254-275.

5. Rudski LG, Lai WW, Afilalo J, Hua L, Handschumacher MD, Chandrasekaran K, Solomon SD, Louie EK, Schiller NB. Guidelines for the echocardiographic assessment of the right heart in adults: a report from the American Society of Echocardiography endorsed by the European Association of Echocardiography, a registered branch of the European Society of Cardiology, and the Canadian Society of Echocardiography. J Am Soc Echocardiogr 2010;23:685-713.

6. Lancellotti P, Tribouilloy C, Hagendorff A, Moura L, Popescu BA, Agricola E, Monin JL, Pierard LA, Badano L, Zamorano JL, European Association of Echocardiography. European Association of Echocardiography recommendations for the assessment of valvular regurgitation. Part 1: aortic and pulmonary regurgitation (native valve disease). Eur J Echocardiogr 2010;11:223-244.

7. Lancellotti P, Moura L, Pierard LA, Agricola E, Popescu BA, Tribouilloy C, Hagendorff A, Monin JL, Badano L, Zamorano JL, European Association of Echocardiography. European Association of Echocardiography recommendations for the assessment of valvular regurgitation. Part 2: mitral and tricuspid regurgitation (native valve disease). Eur J Echocardiogr 2010;11:307-332.

8. Henri C, Pierard LA, Lancellotti P, Mongeon FP, Pibarot P, Basmadjian AJ. Exercise testing and stress imaging in valvular heart disease. Can J Cardiol 2014;30:1012-1026.

9. Picano E, Pibarot P, Lancellotti P, Monin JL, Bonow RO. The emerging role of exercise testing and stress echocardiography in valvular heart disease. J Am Coll Cardiol 2009;54:2251-2260.

10. Monin JL, Quere JP, Monchi M, Petit H, Baleynaud S, Chauvel C, Pop C, Ohlmann P, Lelguen C, Dehant P, Tribouilloy C, Gueret P. Low-gradient aortic stenosis: operative risk stratification and predictors for long-term outcome: a multicenter study using dobutamine stress hemodynamics. Circulation 2003; 108:319-324.

11. Clavel MA, Magne J, Pibarot P. Low-gradient aortic stenosis. Eur Heart J 2016;37:2645-57.

12. American College of Cardiology Foundation Task Force on Expert Consensus Documents, Hundley WG, Bluemke DA, Finn JP, Flamm SD, Fogel MA, Friedrich MG, Ho VB, Jerosch-Herold M, Kramer CM, Manning WJ, Patel M, Pohost GM, Stillman AE, White RD, Woodard PK. ACCF/ACR/AHA/NASCI/ SCMR 2010 expert consensus document on cardiovascular magnetic resonance: a report of the American College of Cardiology Foundation Task Force on Expert Consensus Documents. J Am Coll Cardiol 2010;55:2614-2662.
13. Cueff C, Serfaty JM, Cimadevilla C, Laissy JP, Himbert D, Tubach F, Duval X, lung B, Enriquez-Sarano M, Vahanian A, Messika-Zeitoun D. Measurement of aortic valve calcification using multislice computed tomography: correlation with haemodynamic severity of aortic stenosis and clinical implication for patients with low ejection fraction. Heart 2011;97:721-726.

14. Clavel MA, Messika-Zeitoun D, Pibarot P, Aggarwal SR, Malouf J, Araoz PA Michelena HI, Cueff C, Larose E, Capoulade R, Vahanian A, Enriquez-Sarano M. The complex nature of discordant severe calcified aortic valve disease grading: new insights from combined Doppler echocardiographic and computed tomographic study. J Am Coll Cardiol 2013;62:2329-2338.

15. Bergler-Klein J, Gyongyosi M, Maurer G. The role of biomarkers in valvular heart disease: focus on natriuretic peptides. Can J Cardiol 2014;30:1027-1034.

16. Windecker S, Kolh P, Alfonso F, Collet JP, Cremer J, Falk V, Filippatos G, Hamm C, Head SJ, Juni P, Kappetein AP, Kastrati A, Knuuti J, Landmesser U, Laufer G, Neumann FJ, Richter DJ, Schauerte P, Sousa Uva M, Stefanini GG, Taggart DP, Torracca L, Valgimigli M, Wijns W, Witkowski A. 2014 ESC/ EACTS Guidelines on myocardial revascularization: the Task Force on Myocardial Revascularization of the European Society of Cardiology (ESC) and the European Association for Cardio-Thoracic Surgery (EACTS). Developed with the special contribution of the European Association of Percutaneous Cardiovascular Interventions (EAPCI). Eur Heart J 2014;35:2541-2619.

17. Osnabrugge RL, Speir AM, Head SJ, Fonner CE, Fonner E, Kappetein AP, Rich JB. Performance of EuroSCORE II in a large US database: implications for transcatheter aortic valve implantation. Eur J Cardiothorac Surg 2014;46:400-408.

18. Barili F, Pacini D, Capo A, Rasovic O, Grossi C, Alamanni F, Di Bartolomeo R, Parolari A. Does EuroSCORE II perform better than its original versions? A multicentre validation study. Eur Heart J 2013;34:22-29.

19. lung $B$, Laouenan $C$, Himbert D, Eltchaninoff $H$, Chevreul $K$, Donzeau-Gouge $P$, Fajadet J, Leprince P, Leguerrier A, Lievre M, Prat A, Teiger E, Laskar M, Vahanian A, Gilard M, FRANCE 2 Investigators. Predictive factors of early mortality after transcatheter aortic valve implantation: individual risk assessment using a simple score. Heart 2014;100:1016-1023.

20. Edwards FH, Cohen DJ, O'Brien SM, Peterson ED, Mack MJ, Shahian DM, Grover FL, Tuzcu EM, Thourani VH, Carroll J, Brennan JM, Brindis RG, Rumsfeld J, Holmes DR Jr, Steering Committee of the Society of Thoracic Surgeons/American College of Cardiology Transcatheter Valve Therapy Registry. Development and validation of a risk prediction model for in-hospital mortality after transcatheter aortic valve replacement. JAMA Cardio 2016;1:46-52.

21. Arnold SV, Reynolds MR, Lei Y, Magnuson EA, Kirtane AJ, Kodali SK, Zajarias A, Thourani VH, Green P, Rodes-Cabau J, Beohar N, Mack MJ, Leon MB, Cohen DJ, PARTNER Investigators. Predictors of poor outcomes after transcatheter aortic valve replacement: results from the PARTNER (Placement of Aortic Transcatheter Valve) trial. Circulation 2014;129:2682-2690.

22. Puri R, lung B, Cohen DJ, Rodes-Cabau J. TAVI or no TAVI: identifying patients unlikely to benefit from transcatheter aortic valve implantation. Eur Heart J 2016;37:2217-2225.

23. Gagne JJ, Glynn RJ, Avorn J, Levin R, Schneeweiss S. A combined comorbidity score predicted mortality in elderly patients better than existing scores. J Clin Epidemiol 2011;64:749-759.

24. Gunter RL, Kilgo P, Guyton RA, Chen EP, Puskas JD, Cooper WA, Halkos ME Lattouf OM, Babaliaros V, Myung R, Leshnower B, Thourani VH. Impact of preoperative chronic lung disease on survival after surgical aortic valve replacement. Ann Thorac Surg 2013;96:1322-1328.

25. Allende R, Webb JG, Munoz-Garcia AJ, de jaegere P, Tamburino C, Dager AE, Cheema A, Serra V, Amat-Santos I, Velianou JL, Barbanti M, Dvir D, AlonsoBriales JH, Nuis RJ, Faqiri E, Imme S, Benitez LM, Cucalon AM, Al Lawati H, Garcia del Blanco B, Lopez J, Natarajan MK, DeLarochelliere R, Urena M, Ribeiro HB, Dumont E, Nombela-Franco L, Rodes-Cabau J. Advanced chronic kidney disease in patients undergoing transcatheter aortic valve implantation: insights on clinical outcomes and prognostic markers from a large cohort of patients. Eur Heart J 2014;35:2685-2696.

26. Stortecky S, Schoenenberger AW, Moser A, Kalesan B, Juni P, Carrel T, Bischoff S, Schoenenberger CM, Stuck AE, Windecker S, Wenaweser P. Evaluation of multidimensional geriatric assessment as a predictor of mortality and cardiovascular events after transcatheter aortic valve implantation. JACC Cardiovasc Interv 2012;5:489-496.

27. Puls M, Sobisiak B, Bleckmann A, Jacobshagen C, Danner BC, Hunlich M, Beissbarth T, Schondube F, Hasenfuss G, Seipelt R, Schillinger W. Impact of frailty on short- and long-term morbidity and mortality after transcatheter aortic valve implantation: risk assessment by Katz Index of activities of daily living. Eurolntervention 2014;10:609-619.

28. Habib G, Lancellotti P, Antunes MJ, Bongiorni MG, Casalta JP, Del Zotti F, Dulgheru R, El Khoury G, Erba PA, lung B, Miro JM, Mulder BJ, PlonskaGosciniak E, Price S, Roos-Hesselink J, Snygg-Martin U, Thuny F, Tornos Mas P, Vilacosta I, Zamorano JL, Document R, Erol C, Nihoyannopoulos P, Aboyans V, 
Agewall S, Athanassopoulos G, Aytekin S, Benzer W, Bueno H, Broekhuizen L, Carerj S, Cosyns B, De Backer J, De Bonis M, Dimopoulos K, Donal E, Drexel H, Flachskampf FA, Hall R, Halvorsen S, Hoen B, Kirchhof P, Lainscak M, Leite-Moreira AF, Lip GY, Mestres CA, Piepoli MF, Punjabi PP, Rapezzi C, Rosenhek R, Siebens K, Tamargo J, Walker DM. 2015 ESC Guidelines for the management of infective endocarditis: the Task Force for the Management of Infective Endocarditis of the European Society of Cardiology (ESC). Endorsed by: European Association for Cardio-Thoracic Surgery (EACTS), the European Association of Nuclear Medicine (EANM). Eur Heart J 2015;36:3075-3128.

29. Marijon E, Mirabel M, Celermajer DS, Jouven X. Rheumatic heart disease. Lancet 2012;379:953-964

30. Gerber MA, Baltimore RS, Eaton CB, Gewitz M, Rowley AH, Shulman ST, Taubert KA. Prevention of rheumatic fever and diagnosis and treatment of acute Streptococcal pharyngitis: a scientific statement from the American Heart Association Rheumatic Fever, Endocarditis, and Kawasaki Disease Committee of the Council on Cardiovascular Disease in the Young, the Interdisciplinary Council on Functional Genomics and Translational Biology, and the Interdisciplinary Council on Quality of Care and Outcomes Research: endorsed by the American Academy of Pediatrics. Circulation 2009;119:1541-1551.

31. Remenyi B, Carapetis J, Wyber R, Taubert K, Mayosi BM, World Heart Federation. Position statement of the World Heart Federation on the prevention and control of rheumatic heart disease. Nat Rev Cardiol 2013;10:284-292.

32. Chambers J, Prendergast B, lung B, Rosenhek R, Zamorano JL, Pierard LA, Modine T, Falk V, Kappetein AP, Pibarot P, Sundt T, Bamgartner H, Bax J], Lancellotti P. Standards defining a "heart valve centre": ESC Working Group on Valvular Heart Disease and European Association for Cardiothoracic Surgery viewpoint. Eur Heart J 2017;38:2177-2182.

33. Chambers JB, Ray S, Prendergast B, Taggart D, Westaby S, Grothier L, Arden C, Wilson J, Campbell B, Sandoe J, Gohlke-Barwolf C, Mestres CA, Rosenhek R, Otto C. Specialist valve clinics: recommendations from the British Heart Valve Society working group on improving quality in the delivery of care for patients with heart valve disease. Heart 2013;99:1714-1716.

34. Badheka AO, Patel NJ, Panaich SS, Patel SV, Jhamnani S, Singh V, Pant S, Patel N, Patel N, Arora S, Thakkar B, Manvar S, Dhoble A, Patel A, Savani C, Patel J, Chothani A, Savani GT, Deshmukh A, Grines CL, Curtis J, Mangi AA, Cleman $\mathrm{M}$, Forrest JK. Effect of hospital volume on outcomes of transcatheter aortic valve implantation. Am J Cardiol 2015;116:587-594.

35. Kilic A, Shah AS, Conte JV, Baumgartner WA, Yuh DD. Operative outcomes in mitral valve surgery: combined effect of surgeon and hospital volume in a population-based analysis. J Thorac Cardiovasc Surg 2013;146:638-646.

36. Pagano D, Kappetein AP, Sousa-Uva M, Beyersdorf F, Klautz R, Mohr F, Falk V, European Association for Cardio-Thoracic Surgery (EACTS) and the EACTS Quality Improvement Programme. EACTS clinical statement: guidance for the provision of adult cardiac surgery. Eur J Cardiothorac Surg 2016;50: 1006-1009.

37. Kirchhof P, Benussi S, Kotecha D, Ahlsson A, Atar D, Casadei B, Castella M, Diener HC, Heidbuchel H, Hendriks J, Hindricks G, Manolis AS, Oldgren J, Popescu BA, Schotten U, Van Putte B, Vardas P. 2016 ESC Guidelines for the management of atrial fibrillation developed in collaboration with EACTS: the Task Force for the management of atrial fibrillation of the European Society of Cardiology (ESC). Developed with the special contribution of the European Heart Rhythm Association (EHRA) of the ESC: endorsed by the European Stroke Organisation (ESO). Eur Heart J 2016;37:2893-2962.

38. Breithardt G, Baumgartner H, Berkowitz SD, Hellkamp AS, Piccini JP, Stevens SR, Lokhnygina Y, Patel MR, Halperin JL, Singer DE, Hankey GJ, Hacke W, Becker RC, Nessel CC, Mahaffey KW, Fox KA, Califf RM, ROCKET AF Steering Committee and Investigators. Clinical characteristics and outcomes with rivaroxaban vs. warfarin in patients with non-valvular atrial fibrillation but underlying native mitral and aortic valve disease participating in the ROCKET AF trial. Eur Heart J 2014;35:3377-3385.

39. Avezum A, Lopes RD, Schulte PJ, Lanas F, Gersh B], Hanna M, Pais P, Erol C, Diaz R, Bahit MC, Bartunek J, De Caterina R, Goto S, Ruzyllo W, Zhu J, Granger $\mathrm{CB}$, Alexander JH. Apixaban in comparison with warfarin in patients with atrial fibrillation and valvular heart disease: findings from the Apixaban for Reduction in Stroke and Other Thromboembolic Events in Atrial Fibrillation (ARISTOTLE) Trial. Circulation 2015;132:624-632.

40. Ezekowitz MD, Nagarakanti R, Noack H, Brueckmann M, Litherland C, Jacobs M, Clemens A, Reilly PA, Connolly SJ, Yusuf S, Wallentin L. Comparison of dabigatran and warfarin in patients with atrial fibrillation and valvular heart disease: the RE-LY Trial (Randomized Evaluation of Long-Term Anticoagulant Therapy). Circulation 2016;134:589-598.

41. De Caterina R, Renda G, Carnicelli AP, Nordio F, Trevisan M, Mercuri MF, Ruff $\mathrm{CT}$, Antman EM, Braunwald E, Giugliano RP. Valvular heart disease patients on edoxaban or warfarin in the ENGAGE AF-TIMI 48 Trial. J Am Coll Cardiol 2017:69:1372-1382.
42. lung B, Rodes-Cabau J. The optimal management of anti-thrombotic therapy after valve replacement: certainties and uncertainties. Eur Heart J 2014;35:2942-2949.

43. Heidbuchel H, Verhamme P, Alings M, Antz M, Diener HC, Hacke W, Oldgren J. Sinnaeve P, Camm Al, Kirchhof P. Updated European Heart Rhythm Association Practical Guide on the use of non-vitamin K antagonist anticoagulants in patients with non-valvular atrial fibrillation. Europace 2015;17:1467-1507.

44. Gillinov AM, Gelijns AC, Parides MK, DeRose JJ Jr, Moskowitz AJ, Voisine P, Ailawadi G, Bouchard D, Smith PK, Mack MJ, Acker MA, Mullen JC, Rose EA, Chang HL, Puskas JD, Couderc JP, Gardner TJ, Varghese R, Horvath KA, Bolling SF, Michler RE, Geller NL, Ascheim DD, Miller MA, Bagiella E, Moquete EG, Williams P, Taddei-Peters WC, O'Gara PT, Blackstone EH, Argenziano M, CTSN Investigators. Surgical ablation of atrial fibrillation during mitral-valve surgery. N Engl J Med 2015;372:1399-1409.

45. Eikelboom JW, Connolly SJ, Brueckmann M, Granger CB, Kappetein AP, Mack MJ, Blatchford J, Devenny K, Friedman J, Guiver K, Harper R, Khder Y, Lobmeyer MT, Maas H, Voigt JU, Simoons ML, Van de Werf F, RE-ALIGN Investigators. Dabigatran versus warfarin in patients with mechanical heart valves. N Engl J Med 2013;369:1206-1214.

46. Tsai YC, Phan K, Munkholm-Larsen S, Tian DH, La Meir M, Yan TD. Surgical left atrial appendage occlusion during cardiac surgery for patients with atrial fibrillation: a meta-analysis. Eur / Cardiothorac Surg 2015;47:847-854.

47. lung B, Baron G, Butchart EG, Delahaye F, Gohlke-Barwolf C, Levang OW, Tornos P, Vanoverschelde JL, Vermeer F, Boersma E, Ravaud P, Vahanian A. A prospective survey of patients with valvular heart disease in Europe: the Euro Heart Survey on Valvular Heart Disease. Eur Heart J 2003;24:1231-1243.

48. le Polain de Waroux JB, Pouleur AC, Goffinet C, Vancraeynest D, Van Dyck M, Robert A, Gerber BL, Pasquet A, El Khoury G, Vanoverschelde JL. Functional anatomy of aortic regurgitation: accuracy, prediction of surgical repairability, and outcome implications of transesophageal echocardiography. Circulation 2007;116:1264-269.

49. Lansac E, Di Centa I, Raoux F, Al Attar N, Acar C, Joudinaud T, Raffoul R. A lesional classification to standardize surgical management of aortic insufficiency towards valve repair. Eur J Cardiothorac Surg 2008;33:872-878.

50. Sambola A, Tornos P, Ferreira-Gonzalez I, Evangelista A. Prognostic value of preoperative indexed end-systolic left ventricle diameter in the outcome after surgery in patients with chronic aortic regurgitation. Am Heart J 2008;155:1114-1120.

51. Marciniak A, Sutherland GR, Marciniak M, Claus P, Bijnens B, Jahangiri $M$. Myocardial deformation abnormalities in patients with aortic regurgitation: a strain rate imaging study. Eur J Echocardiogr 2009;10:112-119.

52. Goldstein SA, Evangelista A, Abbara S, Arai A, Asch FM, Badano LP, Bolen MA, Connolly HM, Cuellar-Calabria H, Czerny M, Devereux RB, Erbel RA, Fattori R, Isselbacher EM, Lindsay JM, McCulloch M, Michelena HI, Nienaber CA, Oh JK, Pepi M, Taylor AJ, Weinsaft JW, Zamorano JL, Dietz H, Eagle K, Elefteriades J, Jondeau G, Rousseau H, Schepens M. Multimodality imaging of diseases of the thoracic aorta in adults: from the American Society of Echocardiography and the European Association of Cardiovascular Imaging: endorsed by the Society of Cardiovascular Computed Tomography and Society for Cardiovascular Magnetic Resonance. J Am Soc Echocardiogr 2015;28:119-182.

53. Lang RM, Badano LP, Mor-Avi V, Afilalo J, Armstrong A, Ernande L, Flachskampf FA, Foster E, Goldstein SA, Kuznetsova T, Lancellotti P, Muraru D, Picard MH, Rietzschel ER, Rudski L, Spencer KT, Tsang W, Voigt JU. Recommendations for cardiac chamber quantification by echocardiography in adults: an update from the American Society of Echocardiography and the European Association of Cardiovascular Imaging. J Am Soc Echocardiogr 2015;28:1-39.e14.

54. Freeman LA, Young PM, Foley TA, Williamson EE, Bruce CJ, Greason KL. CT and MRI assessment of the aortic root and ascending aorta. AJR Am J Roentgenol 2013;200:W581-W592.

55. Amsallem M, Ou P, Milleron O, Henry-Feugeas MC, Detaint D, Arnoult F, Vahanian A, Jondeau G. Comparative assessment of ascending aortic aneurysms in Marfan patients using ECG-gated computerized tomographic angiography versus trans-thoracic echocardiography. Int J Cardiol 2015;184:22-27.

56. Erbel R, Aboyans V, Boileau C, Bossone E, Bartolomeo RD, Eggebrecht H, Evangelista A, Falk V, Frank H, Gaemperli O, Grabenwoger M, Haverich A, lung B, Manolis AJ, Meijboom F, Nienaber CA, Roffi M, Rousseau H, Sechtem U, Sirnes PA, Allmen RS, Vrints CJ, ESC Committee for Practice Guidelines. 2014 ESC Guidelines on the diagnosis and treatment of aortic diseases: Document covering acute and chronic aortic diseases of the thoracic and abdominal aorta of the adult. The Task Force for the Diagnosis and Treatment of Aortic Diseases of the European Society of Cardiology (ESC). Eur Heart J 2014;35:2873-2926.

57. Chaliki HP, Mohty D, Avierinos JF, Scott CG, Schaff HV, Tajik AJ, EnriquezSarano M. Outcomes after aortic valve replacement in patients with severe aortic regurgitation and markedly reduced left ventricular function. Circulation 2002;106:2687-2693. 
58. Tornos P, Sambola A, Permanyer-Miralda G, Evangelista A, Gomez Z, SolerSoler J. Long-term outcome of surgically treated aortic regurgitation: influence of guideline adherence toward early surgery. I Am Coll Cardiol 2006; 47:1012-1017.

59. Jondeau G, Detaint D, Tubach F, Arnoult F, Milleron O, Raoux F, Delorme G, Mimoun L, Krapf L, Hamroun D, Beroud C, Roy C, Vahanian A, Boileau C. Aortic event rate in the Marfan population: a cohort study. Circulation 2012;125:226-232

60. Jondeau G, Ropers J, Regalado E, Braverman A, Evangelista A, Teixedo G, De Backer J, Muino-Mosquera L, Naudion S, Zordan C, Morisaki T, Morisaki H, Von Kodolitsch Y, Dupuis-Girod S, Morris SA, Jeremy R, Odent S, Ades LC, Bakshi M, Holman K, LeMaire S, Milleron O, Langeois M, Spentchian M, Aubart M, Boileau C, Pyeritz R, Milewicz DM, Montalcino Aortic Consortium. International Registry of Patients Carrying TGFBR1 or TGFBR2 Mutations: results of the MAC (Montalcino Aortic Consortium). Circ Cardiovasc Genet 2016;9:548-558.

61. Borger MA, Preston M, Ivanov J, Fedak PW, Davierwala P, Armstrong S, David TE. Should the ascending aorta be replaced more frequently in patients with bicuspid aortic valve disease? J Thorac Cardiovasc Surg 2004;128:677-683.

62. Aicher D, Fries R, Rodionycheva S, Schmidt K, Langer F, Schafers HJ. Aortic valve repair leads to a low incidence of valve-related complications. Eur J Cardiothorac Surg 2010;37:127-132.

63. Vohra HA, Whistance RN, De Kerchove L, Punjabi P, El Khoury G. Valve-preserving surgery on the bicuspid aortic valve. Eur I Cardiothorac Surg 2013;43:888-898.

64. Arabkhani B, Mookhoek A, Di Centa I, Lansac E, Bekkers JA, De Lind Van Wijngaarden R, Bogers AJ, Takkenberg JJ. Reported outcome after valve-sparing aortic root replacement for aortic root aneurysm: a systematic review and meta-analysis. Ann Thorac Surg 2015;100:1126-1131.

65. Lansac E, Di Centa I, Sleilaty G, Lejeune S, Khelil N, Berrebi A, Diakov C, Mankoubi L, Malergue MC, Noghin M, Zannis K, Salvi S, Dervanian P, Debauchez M. Long-term results of external aortic ring annuloplasty for aortic valve repair. Eur J Cardiothorac Surg 2016;50:350-360.

66. Dujardin KS, Enriquez-Sarano M, Schaff HV, Bailey KR, Seward JB, Tajik AJ. Mortality and morbidity of aortic regurgitation in clinical practice. A long-term follow-up study. Circulation 1999;99:1851-1857.

67. Klodas E, Enriquez-Sarano M, Tajik AJ, Mullany CJ, Bailey KR, Seward JB. Optimizing timing of surgical correction in patients with severe aortic regurgitation: role of symptoms. J Am Coll Cardiol 1997;30:746-752.

68. Zendaoui A, Lachance D, Roussel E, Couet J, Arsenault M. Usefulness of carvedilol in the treatment of chronic aortic valve regurgitation. Circ Heart Fail 2011;4:207-213.

69. Elder DH, Wei L, Szwejkowski BR, Libianto R, Nadir A, Pauriah M, Rekhraj S, Lim TK, George J, Doney A, Pringle SD, Choy AM, Struthers AD, Lang CC. The impact of renin-angiotensin-aldosterone system blockade on heart failure outcomes and mortality in patients identified to have aortic regurgitation: a large population cohort study. J Am Coll Cardiol 2011;58:2084-2091.

70. Lacro RV, Dietz HC, Sleeper LA, Yetman AT, Bradley TJ, Colan SD, Pearson GD, Selamet Tierney ES, Levine JC, Atz AM, Benson DW, Braverman AC, Chen S, De Backer J, Gelb BD, Grossfeld PD, Klein GL, Lai WW, Liou A, Loeys BL, Markham LW, Olson AK, Paridon SM, Pemberton VL, Pierpont ME, Pyeritz RE, Radojewski E, Roman MJ, Sharkey AM, Stylianou MP, Wechsler SB, Young LT, Mahony L, Pediatric Heart Network Investigators. Atenolol versus losartan in children and young adults with Marfan's syndrome. N Engl J Med 2014;371:2061-2071.

71. Milleron O, Arnoult F, Ropers J, Aegerter P, Detaint D, Delorme G, Attias D, Tubach F, Dupuis-Girod S, Plauchu H, Barthelet M, Sassolas F, Pangaud N, Naudion S, Thomas-Chabaneix J, Dulac Y, Edouard T, Wolf JE, Faivre L, Odent S, Basquin A, Habib G, Collignon P, Boileau C, Jondeau G. Marfan Sartan: a randomized, double-blind, placebo-controlled trial. Eur Heart J 2015;36:2160-2166.

72. Forteza A, Evangelista A, Sanchez V, Teixido-Tura G, Sanz P, Gutierrez L, Gracia T, Centeno J, Rodriguez-Palomares J, Rufilanchas JJ, Cortina J, FerreiraGonzalez I, Garcia-Dorado D. Efficacy of losartan vs. atenolol for the prevention of aortic dilation in Marfan syndrome: a randomized clinical trial. Eur Heart J 2016;37:978-985.

73. Meijboom LJ, Vos FE, Timmermans J, Boers GH, Zwinderman AH, Mulder BJ. Pregnancy and aortic root growth in the Marfan syndrome: a prospective study. Eur Heart J 2005;26:914-920.

74. McKellar SH, MacDonald RJ, Michelena HI, Connolly HM, Sundt TM 3rd. Frequency of cardiovascular events in women with a congenitally bicuspid aortic valve in a single community and effect of pregnancy on events. Am J Cardiol 2011;107:96-99.

75. Braverman AC, Harris KM, Kovacs RJ, Maron BJ, American Heart Association Electrocardiography and Arrhythmias Committee of Council on Clinical Cardiology, Council on Cardiovascular Disease in Young, Council on
Cardiovascular and Stroke Nursing, Council on Functional Genomics and Translational Biology, American College of Cardiology. Eligibility and disqualification recommendations for competitive athletes with cardiovascular abnormalities: Task Force 7: aortic diseases, including Marfan syndrome: a scientific statement from the American Heart Association and American College of Cardiology. Circulation 2015;132:e303-e309.

76. Pizarro R, Bazzino OO, Oberti PF, Falconi ML, Arias AM, Krauss JG, Cagide AM. Prospective validation of the prognostic usefulness of B-type natriuretic peptide in asymptomatic patients with chronic severe aortic regurgitation. J Am Coll Cardiol 2011;58:1705-1714.

77. Weisenberg D, Omelchenko A, Shapira Y, Vaturi M, Monakier D, Bental T, Sagie A. Mid-term echocardiographic progression of patients with moderate aortic regurgitation: implications for aortic valve surgery. J Heart Valve Dis 2013;22:192-194

78. Levy F, Laurent M, Monin JL, Maillet JM, Pasquet A, Le Tourneau T, PetitEisenmann H, Gori M, Jobic Y, Bauer F, Chauvel C, Leguerrier A, Tribouilloy C. Aortic valve replacement for low-flow/low-gradient aortic stenosis operative risk stratification and long-term outcome: a European multicenter study. J Am Coll Cardiol 2008;51:1466-1472.

79. Hachicha Z, Dumesnil JG, Bogaty P, Pibarot P. Paradoxical low-flow, low-gradient severe aortic stenosis despite preserved ejection fraction is associated with higher afterload and reduced survival. Circulation 2007;115:2856-2864.

80. Clavel MA, Dumesnil JG, Capoulade R, Mathieu P, Senechal M, Pibarot P. Outcome of patients with aortic stenosis, small valve area, and low-flow, lowgradient despite preserved left ventricular ejection fraction. J Am Coll Cardio 2012;60:1259-1267.

81. Clavel MA, Pibarot P, Messika-Zeitoun D, Capoulade R, Malouf J, Aggarval S, Araoz PA, Michelena HI, Cueff C, Larose E, Miller JD, Vahanian A, EnriquezSarano M. Impact of aortic valve calcification, as measured by MDCT, on survival in patients with aortic stenosis: results of an international registry study. Am Coll Cardiol 2014;64:1202-1213.

82. Mehrotra P, Jansen K, Flynn AW, Tan TC, Elmariah S, Picard MH, Hung J. Differential left ventricular remodelling and longitudinal function distinguishes low flow from normal-flow preserved ejection fraction low-gradient severe aortic stenosis. Eur Heart J 2013;34:1906-1914.

83. Tribouilloy C, Rusinaru D, Marechaux S, Castel AL, Debry N, Maizel J, Mentaverri R, Kamel S, Slama M, Levy F. Low-gradient, low-flow severe aortic stenosis with preserved left ventricular ejection fraction: characteristics, outcome, and implications for surgery. J Am Coll Cardiol 2015;65:55-66.

84. Jander N, Minners J, Holme I, Gerdts E, Boman K, Brudi P, Chambers JB, Egstrup K, Kesaniemi YA, Malbecq W, Nienaber CA, Ray S, Rossebo A, Pedersen TR, Skjaerpe T, Willenheimer R, Wachtell K, Neumann F], GohlkeBarwolf C. Outcome of patients with low-gradient "severe" aortic stenosis and preserved ejection fraction. Circulation 2011;123:887-895.

85. Rafique AM, Biner S, Ray I, Forrester JS, Tolstrup K, Siegel RJ. Meta-analysis of prognostic value of stress testing in patients with asymptomatic severe aortic stenosis. Am J Cardiol 2009;104:972-977.

86. Marechaux S, Hachicha Z, Bellouin A, Dumesnil JG, Meimoun P, Pasquet A, Bergeron S, Arsenault M, Le Tourneau T, Ennezat PV, Pibarot P. Usefulness of exercise-stress echocardiography for risk stratification of true asymptomatic patients with aortic valve stenosis. Eur Heart J 2010;31:1390-1397.

87. Zamorano JL, Badano LP, Bruce C, Chan KL, Goncalves A, Hahn RT, Keane MG, La Canna G, Monaghan MJ, Nihoyannopoulos P, Silvestry FE, Vanoverschelde JL, Gillam LD. EAE/ASE recommendations for the use of echocardiography in new transcatheter interventions for valvular heart disease. Eur Heart J 2011;32:2189-2214.

88. Azevedo CF, Nigri M, Higuchi ML, Pomerantzeff PM, Spina GS, Sampaio RO, Tarasoutchi F, Grinberg M, Rochitte CE. Prognostic significance of myocardial fibrosis quantification by histopathology and magnetic resonance imaging in patients with severe aortic valve disease. I Am Coll Cardiol 2010;56:278-287.

89. Bergler-Klein J, Klaar U, Heger M, Rosenhek R, Mundigler G, Gabriel H, Binder T, Pacher R, Maurer G, Baumgartner H. Natriuretic peptides predict symptomfree survival and postoperative outcome in severe aortic stenosis. Circulation 2004;109:2302-2308.

90. Clavel MA, Malouf J, Michelena HI, Suri RM, Jaffe AS, Mahoney DW, EnriquezSarano M. B-type natriuretic peptide clinical activation in aortic stenosis: impact on long-term survival. J Am Coll Cardiol 2014;63:2016-2025.

91. Leon MB, Smith CR, Mack M, Miller DC, Moses JW, Svensson LG, Tuzcu EM, Webb JG, Fontana GP, Makkar RR, Brown DL, Block PC, Guyton RA, Pichard AD, Bavaria JE, Herrmann HC, Douglas PS, Petersen JL, Akin JJ, Anderson WN, Wang D, Pocock S, PARTNER Trial Investigators. Transcatheter aortic-valve implantation for aortic stenosis in patients who cannot undergo surgery. N Eng J Med 2010;363:1597-1607.

92. Rosenhek R, Binder T, Porenta G, Lang I, Christ G, Schemper M, Maurer G, Baumgartner $\mathrm{H}$. Predictors of outcome in severe, asymptomatic aortic stenosis. N Engl J Med 2000;343:611-617. 
93. Thourani VH, Suri RM, Gunter RL, Sheng S, O'Brien SM, Ailawadi G, Szeto WY, Dewey TM, Guyton RA, Bavaria JE, Babaliaros V, Gammie JS, Svensson L, Williams M, Badhwar V, Mack MJ. Contemporary real-world outcomes of surgical aortic valve replacement in 141,905 low-risk, intermediate-risk, and high-risk patients. Ann Thorac Surg 2015;99:55-61.

94. Deeb GM, Reardon MJ, Chetcuti S, Patel HJ, Grossman PM, Yakubov SJ, Kleiman NS, Coselli JS, Gleason TG, Lee JS, Hermiller JB Jr, Heiser J, Merhi W, Zorn GL 3rd, Tadros P, Robinson N, Petrossian G, Hughes GC, Harrison JK, Maini B, Mumtaz M, Conte J, Resar J, Aharonian V, Pfeffer T, Oh JK, Qiao H, Adams DH, Popma J], CoreValve US Clinical Investigators. 3-year outcomes in high-risk patients who underwent surgical or transcatheter aortic valve replacement. J Am Coll Cardiol 2016;67:2565-2574.

95. Smith CR, Leon MB, Mack MJ, Miller DC, Moses JW, Svensson LG, Tuzcu EM, Webb JG, Fontana GP, Makkar RR, Williams M, Dewey T, Kapadia S, Babaliaros V, Thourani VH, Corso P, Pichard AD, Bavaria JE, Herrmann HC, Akin J], Anderson WN, Wang D, Pocock SJ, PARTNER Trial Investigators. Transcatheter versus surgical aortic-valve replacement in high-risk patients. N Engl J Med 2011;364:2187-2198.

96. Mack MJ, Leon MB, Smith CR, Miller DC, Moses JW, Tuzcu EM, Webb JG, Douglas PS, Anderson WN, Blackstone EH, Kodali SK, Makkar RR, Fontana GP, Kapadia S, Bavaria J, Hahn RT, Thourani VH, Babaliaros V, Pichard A, Herrmann HC, Brown DL, Williams M, Akin J, Davidson MJ, Svensson LG, PARTNER 1 Trial Investigators. 5-year outcomes of transcatheter aortic valve replacement or surgical aortic valve replacement for high surgical risk patients with aortic stenosis (PARTNER 1): a randomised controlled trial. Lancet 2015;385:2477-2484.

97. Adams DH, Popma J], Reardon MJ, Yakubov SJ, Coselli JS, Deeb GM, Gleason TG, Buchbinder M, Hermiller J Jr, Kleiman NS, Chetcuti S, Heiser J, Merhi W, Zorn G, Tadros P, Robinson N, Petrossian G, Hughes GC, Harrison JK, Conte J, Maini B, Mumtaz M, Chenoweth S, Oh JK, U.S. CoreValve Clinical Investigators. Transcatheter aortic-valve replacement with a self-expanding prosthesis. N Engl J Med 2014;370:1790-1798.

98. Thyregod HG, Steinbruchel DA, Ihlemann N, Nissen H, Kjeldsen BJ, Petursson $P$, Chang Y, Franzen OW, Engstrom T, Clemmensen P, Hansen PB, Andersen LW, Olsen PS, Sondergaard L. Transcatheter versus surgical aortic valve replacement in patients with severe aortic valve stenosis: 1-year results from the all-comers NOTION randomized clinical trial. J Am Coll Cardiol 2015;65:2184-2194.

99. Leon MB, Smith CR, Mack MJ, Makkar RR, Svensson LG, Kodali SK, Thourani VH, Tuzcu EM, Miller DC, Herrmann HC, Doshi D, Cohen DJ, Pichard AD, Kapadia S, Dewey T, Babaliaros V, Szeto WY, Williams MR, Kereiakes D, Zajarias A, Greason KL, Whisenant BK, Hodson RW, Moses JW, Trento A, Brown DL, Fearon WF, Pibarot P, Hahn RT, Jaber WA, Anderson WN, Alu MC, Webb JG, PARTNER 2 Investigators. Transcatheter or surgical aortic-valve replacement in intermediate-risk patients. N Engl J Med 2016;374:1609-1620.

100. Thourani VH, Kodali S, Makkar RR, Herrmann HC, Williams M, Babaliaros V, Smalling R, Lim S, Malaisrie SC, Kapadia S, Szeto WY, Greason KL, Kereiakes D, Ailawadi G, Whisenant BK, Devireddy C, Leipsic J, Hahn RT, Pibarot P, Weissman NJ, Jaber WA, Cohen DJ, Suri R, Tuzcu EM, Svensson LG, Webb JG, Moses JW, Mack MJ, Miller DC, Smith CR, Alu MC, Parvataneni R, D'Agostino $\mathrm{RB} J \mathrm{~J}$, Leon MB. Transcatheter aortic valve replacement versus surgical valve replacement in intermediate-risk patients: a propensity score analysis. Lancet 2016;387:2218-2225.

101. Siontis GC, Praz F, Pilgrim T, Mavridis D, Verma S, Salanti G, Sondergaard L, Juni $P$, Windecker $S$. Transcatheter aortic valve implantation vs. surgical aortic valve replacement for treatment of severe aortic stenosis: a meta-analysis of randomized trials. Eur Heart J 2016;37:3503-3512.

102. Reardon MJ, Van Mieghem NM, Popma JJ, Kleiman NS, Sondergaard L, Mumtaz M, Adams DH, Deeb GM, Maini B, Gada H, Chetcuti S, Gleason T, Heiser J, Lange R, Merhi W, Oh JK, Olsen PS, Piazza N, Williams M, Windecker S, Yakubov SJ, Grube E, Makkar R, Lee JS, Conte J, Vang E, Nguyen H, Chang Y, Mugglin AS, Serruys PW, Kappetein AP, SURTAVI Investigators. Surgical or transcatheter aortic-valve replacement in intermediate-risk patients. N Engl J Med 2017;376:1321-1331.

103. Rogers T, Koifman E, Patel N, Gai J, Torguson R, Corso P, Waksman R. Society of Thoracic Surgeons score variance results in risk reclassification of patients undergoing transcatheter aortic valve replacement. JAMA Cardiol 2017;2:455-456.

104. Tribouilloy C, Levy F, Rusinaru D, Gueret P, Petit-Eisenmann H, Baleynaud S, Jobic Y, Adams C, Lelong B, Pasquet A, Chauvel C, Metz D, Quere JP, Monin JL. Outcome after aortic valve replacement for low-flow/low-gradient aortic stenosis without contractile reserve on dobutamine stress echocardiography. J Am Coll Cardiol 2009;53:1865-1873.

105. Fougeres E, Tribouilloy C, Monchi M, Petit-Eisenmann H, Baleynaud S, Pasquet A, Chauvel C, Metz D, Adams C, Rusinaru D, Gueret P, Monin JL. Outcomes of pseudo-severe aortic stenosis under conservative treatment. Eur Heart J 2012;33:2426-2433.
106. Genereux P, Stone GW, O'Gara PT, Marquis-Gravel G, Redfors B, Giustino G, Pibarot P, Bax JJ, Bonow RO, Leon MB. Natural history, diagnostic approaches, and therapeutic strategies for patients with asymptomatic severe aortic stenosis. J Am Coll Cardiol 2016;67:2263-2288.

107. Das $\mathrm{P}$, Rimington $\mathrm{H}$, Chambers J. Exercise testing to stratify risk in aortic stenosis. Eur Heart J 2005;26:1309-1313.

108. Rosenhek R, Zilberszac R, Schemper M, Czerny M, Mundigler G, Graf S, Bergler-Klein J, Grimm M, Gabriel H, Maurer G. Natural history of very severe aortic stenosis. Circulation 2010;121:151-156.

109. Cioffi G, Faggiano P, Vizzardi E, Tarantini L, Cramariuc D, Gerdts E, de Simone G. Prognostic effect of inappropriately high left ventricular mass in asymptomatic severe aortic stenosis. Heart 2011;97:301-307.

110. Dahl JS, Videbaek L, Poulsen MK, Rudbaek TR, Pellikka PA, Moller JE. Global strain in severe aortic valve stenosis: relation to clinical outcome after aortic valve replacement. Circ Cardiovasc Imaging 2012;5:613-620.

111. Zlotnick DM, Ouellette ML, Malenka DJ, DeSimone JP, Leavitt BJ, Helm RE, Olmstead EM, Costa SP, DiScipio AW, Likosky DS, Schmoker JD, Quinn RD, Sisto D, Klemperer JD, Sardella GL, Baribeau YR, Frumiento C, Brown JR, O'Rourke DJ, Northern New England Cardiovascular Disease Study Group. Effect of preoperative pulmonary hypertension on outcomes in patients with severe aortic stenosis following surgical aortic valve replacement. Am J Cardiol 2013;112:1635-1640.

112. Rossebo AB, Pedersen TR, Boman K, Brudi P, Chambers JB, Egstrup K, Gerdts E, Gohlke-Barwolf C, Holme I, Kesaniemi YA, Malbecq W, Nienaber CA, Ray S, Skjaerpe T, Wachtell K, Willenheimer R, SEAS Investigators. Intensive lipid lowering with simvastatin and ezetimibe in aortic stenosis. N Engl J Med 2008;359:1343-1356.

113. Ponikowski P, Voors AA, Anker SD, Bueno H, Cleland JG, Coats AJ, Falk V, Gonzalez-Juanatey JR, Harjola VP, Jankowska EA, Jessup M, Linde C, Nihoyannopoulos P, Parissis JT, Pieske B, Riley JP, Rosano GM, Ruilope LM, Ruschitzka F, Rutten FH, van der Meer P. 2016 ESC Guidelines for the diagnosis and treatment of acute and chronic heart failure: the Task Force for the diagnosis and treatment of acute and chronic heart failure of the European Society of Cardiology (ESC). Developed with the special contribution of the Heart Failure Association (HFA) of the ESC. Eur Heart J 2016;37:2129-2200.

114. Smith WT 4th, Ferguson TB Jr, Ryan T, Landolfo CK, Peterson ED. Should coronary artery bypass graft surgery patients with mild or moderate aortic stenosis undergo concomitant aortic valve replacement? A decision analysis approach to the surgical dilemma. J Am Coll Cardiol 2004;44:1241-1247.

115. Baumgartner H, Bonhoeffer P, De Groot NM, de Haan F, Deanfield JE, Galie N, Gatzoulis MA, Gohlke-Baerwolf C, Kaemmerer H, Kilner P, Meijboom F, Mulder BJ, Oechslin E, Oliver JM, Serraf A, Szatmari A, Thaulow E, Vouhe PR, Walma E, Task Force on the Management of Grown-up Congenital Heart Disease of the European Society of Cardiology (ESC), Association for European Paediatric Cardiology (AEPC), ESC Committee for Practice Guidelines (CPG). ESC Guidelines for the management of grown-up congenital heart disease (new version 2010). Eur Heart J 2010;31:2915-2957.

116. De Bonis M, Al-Attar N, Antunes M, Borger M, Casselman F, Falk V, Folliguet T, lung B, Lancellotti P, Lentini S, Maisano F, Messika-Zeitoun D, Muneretto C, Pibarot P, Pierard L, Punjabi P, Rosenhek R, Suwalski P, Vahanian A, Wendler $O$, Prendergast $B$. Surgical and interventional management of mitral valve regurgitation: a position statement from the European Society of Cardiology Working Groups on Cardiovascular Surgery and Valvular Heart Disease. Eur Heart J 2016;37:133-139.

117. Monin JL, Dehant P, Roiron C, Monchi M, Tabet JY, Clerc P, Fernandez G, Houel R, Garot J, Chauvel C, Gueret P. Functional assessment of mitral regurgitation by transthoracic echocardiography using standardized imaging planes diagnostic accuracy and outcome implications. J Am Coll Cardiol 2005;46:302-309.

118. Lancellotti P, Gerard PL, Pierard LA. Long-term outcome of patients with heart failure and dynamic functional mitral regurgitation. Eur Heart J 2005; 26:1528-1532

119. Magne J, Lancellotti P, Pierard LA. Exercise pulmonary hypertension in asymptomatic degenerative mitral regurgitation. Circulation 2010;122:33-41.

120. Pizarro R, Bazzino OO, Oberti PF, Falconi M, Achilli F, Arias A, Krauss JG, Cagide AM. Prospective validation of the prognostic usefulness of brain natriuretic peptide in asymptomatic patients with chronic severe mitral regurgitation. J Am Coll Cardiol 2009;54:1099-1106.

121. Tribouilloy CM, Enriquez-Sarano M, Schaff HV, Orszulak TA, Bailey KR, Tajik AJ, Frye RL. Impact of preoperative symptoms on survival after surgical correction of organic mitral regurgitation: rationale for optimizing surgical indications. Circulation 1999;99:400-405.

122. Enriquez-Sarano M, Tajik AJ, Schaff HV, Orszulak TA, Bailey KR, Frye RL. Echocardiographic prediction of survival after surgical correction of organic mitral regurgitation. Circulation 1994;90:830-837. 
123. Badhwar V, Peterson ED, Jacobs JP, He X, Brennan JM, O'Brien SM, Dokholyan RS, George KM, Bolling SF, Shahian DM, Grover FL, Edwards FH, Gammie JS. Longitudinal outcome of isolated mitral repair in older patients: results from 14,604 procedures performed from 1991 to 2007. Ann Thorac Surg 2012;94:1870-1877; discussion 1877-1879.

124. Le Tourneau T, Richardson M, Juthier F, Modine T, Fayad G, Polge AS, Ennezat PV, Bauters C, Vincentelli A, Deklunder G. Echocardiography predictors and prognostic value of pulmonary artery systolic pressure in chronic organic mitral regurgitation. Heart 2010;96:1311-1317.

125. Tribouilloy C, Grigioni F, Avierinos JF, Barbieri A, Rusinaru D, Szymanski C, Ferlito M, Tafanelli L, Bursi F, Trojette F, Branzi A, Habib G, Modena MG, Enriquez-Sarano M, MIDA Investigators. Survival implication of left ventricular end-systolic diameter in mitral regurgitation due to flail leaflets a long-term follow-up multicenter study. J Am Coll Cardiol 2009;54:1961-1968.

126. Rosenhek R, Rader F, Klaar U, Gabriel H, Krejc M, Kalbeck D, Schemper M, Maurer G, Baumgartner $\mathrm{H}$. Outcome of watchful waiting in asymptomatic severe mitral regurgitation. Circulation 2006;113:2238-2244.

127. Gammie JS, O'Brien SM, Griffith BP, Ferguson TB, Peterson ED. Influence of hospital procedural volume on care process and mortality for patients undergoing elective surgery for mitral regurgitation. Circulation 2007;115:881-887.

128. Bolling SF, Li S, O'Brien SM, Brennan JM, Prager RL, Gammie JS. Predictors of mitral valve repair: clinical and surgeon factors. Ann Thorac Surg 2010;90:1904-1911.

129. Feldman T, Foster E, Glower DD, Kar S, Rinaldi MJ, Fail PS, Smalling RW, Siegel R, Rose GA, Engeron E, Loghin C, Trento A, Skipper ER, Fudge T, Letsou GV, Massaro JM, Mauri L, EVEREST II Investigators. Percutaneous repair or surgery for mitral regurgitation. N Engl J Med 2011;364:1395-1406.

130. Feldman T, Kar S, Elmariah S, Smart SC, Trento A, Siegel RJ, Apruzzese P, Fail P, Rinaldi MJ, Smalling RW, Hermiller JB, Heimansohn D, Gray WA, Grayburn PA, Mack MJ, Lim DS, Ailawadi G, Herrmann HC, Acker MA, Silvestry FE, Foster E, Wang A, Glower DD, Mauri L, EVEREST II Investigators. Randomized comparison of percutaneous repair and surgery for mitral regurgitation: 5-year results of EVEREST II. J Am Coll Cardiol 2015;66:2844-2854.

131. Enriquez-Sarano M, Tajik AJ, Schaff HV, Orszulak TA, McGoon MD, Bailey KR, Frye RL. Echocardiographic prediction of left ventricular function after correction of mitral regurgitation: results and clinical implications. J Am Coll Cardiol 1994;24:1536-1543.

132. Haan CK, Cabral Cl, Conetta DA, Coombs LP, Edwards FH. Selecting patients with mitral regurgitation and left ventricular dysfunction for isolated mitral valve surgery. Ann Thorac Surg 2004;78:820-825.

133. Samad Z, Kaul P, Shaw LK, Glower DD, Velazquez EJ, Douglas PS, Jollis JG. Impact of early surgery on survival of patients with severe mitral regurgitation. Heart 2011;97:221-224.

134. Levine RA, Schwammenthal E. Ischemic mitral regurgitation on the threshold of a solution: from paradoxes to unifying concepts. Circulation 2005;112:745-758.

135. Grigioni F, Enriquez-Sarano M, Zehr KJ, Bailey KR, Tajik AJ. Ischemic mitral regurgitation: long-term outcome and prognostic implications with quantitative Doppler assessment. Circulation 2001;103:1759-1764.

136. Acker MA, Parides MK, Perrault LP, Moskowitz AJ, Gelijns AC, Voisine P, Smith PK, Hung JW, Blackstone EH, Puskas JD, Argenziano M, Gammie JS, Mack M, Ascheim DD, Bagiella E, Moquete EG, Ferguson TB, Horvath KA, Geller NL, Miller MA, Woo YJ, D'Alessandro DA, Ailawadi G, Dagenais F, Gardner TJ, O'Gara PT, Michler RE, Kron IL. Mitral-valve repair versus replacement for severe ischemic mitral regurgitation. N Engl J Med 2014;370:23-32.

137. Mihaljevic T, Lam BK, Rajeswaran J, Takagaki M, Lauer MS, Gillinov AM, Blackstone EH, Lytle BW. Impact of mitral valve annuloplasty combined with revascularization in patients with functional ischemic mitral regurgitation. J Am Coll Cardiol 2007;49:2191-2201.

138. Wu AH, Aaronson KD, Bolling SF, Pagani FD, Welch K, Koelling TM. Impact of mitral valve annuloplasty on mortality risk in patients with mitral regurgitation and left ventricular systolic dysfunction. J Am Coll Cardiol 2005;45:381-387.

139. Mauri L, Foster E, Glower DD, Apruzzese P, Massaro JM, Herrmann HC, Hermiller J, Gray W, Wang A, Pedersen WR, Bajwa T, Lasala J, Low R, Grayburn P, Feldman T, EVEREST II Investigators. 4-year results of a randomized controlled trial of percutaneous repair versus surgery for mitral regurgitation. J Am Coll Cardiol 2013;62:317-328.

140. Maisano F, Franzen O, Baldus S, Schafer U, Hausleiter J, Butter C, Ussia GP, Sievert H, Richardt G, Widder JD, Moccetti T, Schillinger W. Percutaneous mitral valve interventions in the real world: early and 1-year results from the ACCESS-EU, a prospective, multicenter, nonrandomized postapproval study of the MitraClip therapy in Europe. J Am Coll Cardiol 2013;62: 1052-1061.

141. Michler RE, Smith PK, Parides MK, Ailawadi G, Thourani V, Moskowitz AJ, Acker MA, Hung JW, Chang HL, Perrault LP, Gillinov AM, Argenziano M, Bagiella E, Overbey JR, Moquete EG, Gupta LN, Miller MA, Taddei-Peters WC, Jeffries N, Weisel RD, Rose EA, Gammie JS, DeRose JJ Jr, Puskas JD, Dagenais F,
Burks SG, El-Hamamsy I, Milano CA, Atluri P, Voisine P, O'Gara PT, Gelijns AC, CTSN. Two-year outcomes of surgical treatment of moderate ischemic mitral regurgitation. N Engl J Med 2016;374:1932-1941.

142. lung B, Vahanian A. Epidemiology of acquired valvular heart disease. Can J Cardiol 2014;30:962-970.

143. Abramowitz Y, Jilaihawi H, Chakravarty T, Mack MJ, Makkar RR. Mitral annulus calcification. J Am Coll Cardiol 2015;66:1934-1941.

144. Bouleti C, lung B, Laouenan C, Himbert D, Brochet E, Messika-Zeitoun D, Detaint D, Garbarz E, Cormier B, Michel PL, Mentre F, Vahanian A. Late results of percutaneous mitral commissurotomy up to 20 years: development and validation of a risk score predicting late functional results from a series of 912 patients. Circulation 2012;125:2119-2127.

145. Wilkins GT, Weyman AE, Abascal VM, Block PC, Palacios IF. Percutaneous balloon dilatation of the mitral valve: an analysis of echocardiographic variables related to outcome and the mechanism of dilatation. $\mathrm{Br}$ Heart J 1988;60:299-308.

146. Nunes MC, Tan TC, Elmariah S, do Lago R, Margey R, Cruz-Gonzalez I, Zheng $\mathrm{H}$, Handschumacher MD, Inglessis I, Palacios IF, Weyman AE, Hung J. The echo score revisited: Impact of incorporating commissural morphology and leaflet displacement to the prediction of outcome for patients undergoing percutaneous mitral valvuloplasty. Circulation 2014;129:886-895.

147. Badheka AO, Shah N, Ghatak A, Patel NJ, Chothani A, Mehta K, Singh V, Patel N, Grover P, Deshmukh A, Panaich SS, Savani GT, Bhalara V, Arora S, Rathod A Desai H, Kar S, Alfonso C, Palacios IF, Grines C, Schreiber T, Rihal CS, Makkar R, Cohen MG, O'Neill W, de Marchena E. Balloon mitral valvuloplasty in the United States: a 13-year perspective. Am J Med 2014;127:1126 e1121-1112.

148. Tomai F, Gaspardone A, Versaci F, Ghini AS, Altamura L, De Luca L, Gioffre G, Gioffre PA. Twenty year follow-up after successful percutaneous balloon mitral valvuloplasty in a large contemporary series of patients with mitral stenosis. Int J Cardiol 2014;177:881-885.

149. Bouleti C, lung B, Himbert D, Messika-Zeitoun D, Brochet E, Garbarz E, Cormier B, Vahanian A. Relationship between valve calcification and long-term results of percutaneous mitral commissurotomy for rheumatic mitral stenosis. Circ Cardiovasc Interv 2014;7:381-389.

150. lung B, Cormier B, Ducimetiere P, Porte JM, Nallet O, Michel PL, Acar J, Vahanian A. Immediate results of percutaneous mitral commissurotomy. A predictive model on a series of 1514 patients. Circulation 1996;94:2124-2130.

151. Bouleti C, lung B, Himbert D, Brochet E, Messika-Zeitoun D, Detaint D Garbarz E, Cormier B, Vahanian A. Reinterventions after percutaneous mitral commissurotomy during long-term follow-up, up to 20 years: the role of repeat percutaneous mitral commissurotomy. Eur Heart J 2013;34:1923-1930.

152. Guerrero M, Dvir D, Himbert D, Urena M, Eleid M, Wang DD, Greenbaum A, Mahadevan VS, Holzhey D, O'Hair D, Dumonteil N, Rodes-Cabau J, Piazza N Palma JH, Delago A, Ferrari E, Witkowski A, Wendler O, Kornowski R, Martinez-Clark P, Ciaburri D, Shemin R, Alnasser S, McAllister D, Bena M, Kerendi F, Pavlides G, Sobrinho JJ, Attizzani GF, George I, Nickenig G, Fassa AA, Cribier A, Bapat V, Feldman T, Rihal C, Vahanian A, Webb J, O'Neill W. Transcatheter mitral valve replacement in native mitral valve disease with severe mitral annular calcification: results from the first multicenter global registry. JACC Cardiovasc Interv 2016;9:1361-1371.

153. Song $H$, Kang DH, Kim JH, Park KM, Song JM, Choi KJ, Hong MK, Chung $\mathrm{CH}$ Song JK, Lee JW, Park SW, Park SJ. Percutaneous mitral valvuloplasty versus surgical treatment in mitral stenosis with severe tricuspid regurgitation. Circulation 2007;116:I-246-I-250.

154. Sousa C, Botelho C, Rodrigues D, Azeredo J, Oliveira R. Infective endocarditis in intravenous drug abusers: an update. Eur J Clin Microbiol Infect Dis 2012;31:2905-2910.

155. Park JB, Lee SP, Lee JH, Yoon YE, Park EA, Kim HK, Lee W, Kim YJ, Cho GY, Sohn DW. Quantification of right ventricular volume and function using singlebeat three-dimensional echocardiography: a validation study with cardiac magnetic resonance. J Am Soc Echocardiogr 2016;29:392-401.

156. Dreyfus GD, Corbi PJ, Chan KM, Bahrami T. Secondary tricuspid regurgitation or dilatation: which should be the criteria for surgical repair? Ann Thorac Surg 2005;79:127-132.

157. Van de Veire NR, Braun J, Delgado V, Versteegh MI, Dion RA, Klautz RJ, Bax JJ. Tricuspid annuloplasty prevents right ventricular dilatation and progression of tricuspid regurgitation in patients with tricuspid annular dilatation undergoing mitral valve repair. J Thorac Cardiovasc Surg 2011;141:1431-1439.

158. Nath J, Foster E, Heidenreich PA. Impact of tricuspid regurgitation on longterm survival. J Am Coll Cardiol 2004;43:405-409.

159. Kammerlander AA, Marzluf BA, Graf A, Bachmann A, Kocher A, Bonderman D, Mascherbauer J. Right ventricular dysfunction, but not tricuspid regurgitation, is associated with outcome late after left heart valve procedure. J Am Coll Cardiol 2014:64:2633-2642.

160. Chikwe J, Itagaki S, Anyanwu A, Adams DH. Impact of concomitant tricuspid annuloplasty on tricuspid regurgitation, right ventricular function, and 
pulmonary artery hypertension after repair of mitral valve prolapse. J Am Coll Cardiol 2015;65:1931-1938.

161. Chang BC, Lim SH, Yi G, Hong YS, Lee S, Yoo KJ, Kang MS, Cho BK. Long-term clinical results of tricuspid valve replacement. Ann Thorac Surg 2006;81:1317-1323; discussion 1323-1314.

162. Filsoufi F, Anyanwu AC, Salzberg SP, Frankel T, Cohn LH, Adams DH. Longterm outcomes of tricuspid valve replacement in the current era. Ann Thorac Surg 2005;80:845-850.

163. Yeter E, Ozlem K, Kilic H, Ramazan A, Acikel S. Tricuspid balloon valvuloplasty to treat tricuspid stenosis. J Heart Valve Dis 2010;19:159-160.

164. Unger P, Rosenhek R, Dedobbeleer C, Berrebi A, Lancellotti P. Management of multiple valve disease. Heart 2011;97:272-277.

165. Hammermeister K, Sethi GK, Henderson WG, Grover FL, Oprian C, Rahimtoola SH. Outcomes 15 years after valve replacement with a mechanical versus a bioprosthetic valve: final report of the Veterans Affairs randomized trial. J Am Coll Cardiol 2000;36:1152-1158.

166. Oxenham H, Bloomfield P, Wheatley DJ, Lee RJ, Cunningham J, Prescott RJ, Miller HC. Twenty year comparison of a Bjork-Shiley mechanical heart valve with porcine bioprostheses. Heart 2003;89:715-721.

167. Stassano P, Di Tommaso L, Monaco M, lorio F, Pepino P, Spampinato N, Vosa C. Aortic valve replacement: a prospective randomized evaluation of mechanical versus biological valves in patients ages 55 to 70 years. J Am Coll Cardiol 2009;54:1862-1868.

168. Capodanno D, Petronio AS, Prendergast B, Eltchaninoff $\mathrm{H}$, Vahanian A, Modine T, Lancellotti P, Sondergaard L, Ludman PF, Tamburimno C, Piazza N, Hancock J, Mehilli J, Byrne RA, Baumbach A, Kappetein AP, Windecker S, Bax J, Haude M. Standardised definitions of structural deterioration and valve failure in assessing long-term durability of transcatheter and surgical aortic bioprosthetic valves - a consensus statement from the European Association of Percutaneous Cardiovascular Interventions (EAPCI) endorsed by the European Society of Cardiology (ESC) and the European Association for Cardio-Thoracic Surgery (EACTS). Eur Heart J 2017; DOl:https://doi.org/10.1093/eurheartj/eh×303.

169. Zoghbi WA, Chambers JB, Dumesnil JG, Foster E, Gottdiener JS, Grayburn PA, Khandheria BK, Levine RA, Marx GR, Miller FA Jr, Nakatani S, Quinones MA, Rakowski H, Rodriguez LL, Swaminathan M, Waggoner AD, Weissman NJ, Zabalgoitia M, American Society of Echocardiography's Guidelines and Standards Committee, Task Force on Prosthetic Valves, American College of Cardiology Cardiovascular Imaging Committee, Cardiac Imaging Committee of the American Heart Association, European Association of Echocardiography, European Society of Cardiology, Japanese Society of Echocardiography, Canadian Society of Echocardiography, American College of Cardiology Foundation, American Heart Association, European Association of Echocardiography, European Society of Cardiology, Japanese Society of Echocardiography, Canadian Society of Echocardiography. Recommendations for evaluation of prosthetic valves with echocardiography and Doppler ultrasound: a report from the American Society of Echocardiography's Guidelines and Standards Committee and the Task Force on Prosthetic Valves, developed in conjunction with the American College of Cardiology Cardiovascular Imaging Committee, Cardiac Imaging Committee of the American Heart Association, the European Association of Echocardiography, a registered branch of the European Society of Cardiology, the Japanese Society of Echocardiography and the Canadian Society of Echocardiography, endorsed by the American College of Cardiology Foundation, American Heart Association, European Association of Echocardiography, a registered branch of the European Society of Cardiology, the Japanese Society of Echocardiography, and Canadian Society of Echocardiography. J Am Soc Echocardiogr 2009;22:975-1014; quiz 1082-1014.

170. Lancellotti P, Pibarot P, Chambers J, Edvardsen T, Delgado V, Dulgheru R, Pepi M, Cosyns B, Dweck MR, Garbi M, Magne J, Nieman K, Rosenhek R, Bernard A, Lowenstein J, Vieira ML, Rabischoffsky A, Vyhmeister RH, Zhou X, Zhang Y, Zamorano JL, Habib G. Recommendations for the imaging assessment of prosthetic heart valves: a report from the European Association of Cardiovascular Imaging endorsed by the Chinese Society of Echocardiography, the InterAmerican Society of Echocardiography, and the Brazilian Department of Cardiovascular Imaging. Eur Heart J Cardiovasc Imaging 2016;17:589-590.

171. Butchart EG, Gohlke-Barwolf C, Antunes MJ, Tornos P, De Caterina R, Cormier B, Prendergast B, lung B, Bjornstad H, Leport C, Hall RJ, Vahanian A, Working Groups on Valvular Heart Disease, Thrombosis, and Cardiac Rehabilitation and Exercise Physiology, European Society of Cardiology. Recommendations for the management of patients after heart valve surgery. Eur Heart J 2005;26:2463-2471.

172. Brennan JM, Edwards FH, Zhao Y, O'Brien S, Booth ME, Dokholyan RS, Douglas PS, Peterson ED, DEcIDE AVR Research Team. Early anticoagulation of bioprosthetic aortic valves in older patients: results from the Society of Thoracic Surgeons Adult Cardiac Surgery National Database. J Am Coll Cardiol 2012;60:971-977.
173. Merie C, Kober L, Skov Olsen P, Andersson C, Gislason G, Skov Jensen J, Torp-Pedersen C. Association of warfarin therapy duration after bioprosthetic aortic valve replacement with risk of mortality, thromboembolic complications, and bleeding. JAMA 2012;308:2118-2125.

174. Rivas-Gandara N, Ferreira-Gonzalez I, Tornos P, Torrents A, PermanyerMiralda G, Nicolau I, Arellano-Rodrigo E, Vallejo N, Igual A, Soler-Soler J. Enoxaparin as bridging anticoagulant treatment in cardiac surgery. Heart 2008;94:205-210.

175. Meurin P, Tabet JY, Weber H, Renaud N, Ben Driss A. Low-molecular-weight heparin as a bridging anticoagulant early after mechanical heart valve replacement. Circulation 2006;113:564-569.

176. Laffort P, Roudaut R, Roques X, Lafitte S, Deville C, Bonnet J, Baudet E. Early and long-term (one-year) effects of the association of aspirin and oral anticoagulant on thrombi and morbidity after replacement of the mitral valve with the St. Jude medical prosthesis: a clinical and transesophageal echocardiographic study. J Am Coll Cardiol 2000;35:739-746.

177. Vavuranakis M, Siasos G, Zografos T, Oikonomou E, Vrachatis D, Kalogeras K, Papaioannou T, Kolokathis MA, Moldovan C, Tousoulis D. Dual or single antiplatelet therapy after transcatheter aortic valve implantation? A systematic review and meta-analysis. Curr Pharm Des 2016;22:4596-4603.

178. Chakravarty T, Sondergaard L, Friedman J, De Backer O, Berman D, Kofoed KF, Jilaihawi H, Shiota T, Abramowitz Y, Jorgensen TH, Rami T, Israr S, Fontana G, de Knegt M, Fuchs A, Lyden P, Trento A, Bhatt DL, Leon MB, Makkar RR, RESOLVE, SAVORY Investigators. Subclinical leaflet thrombosis in surgical and transcatheter bioprosthetic aortic valves: an observational study. Lancet 2017;389:2383-2392.

179. Cannegieter SC, Rosendaal FR, Briet E. Thromboembolic and bleeding complications in patients with mechanical heart valve prostheses. Circulation 1994;89:635-641.

180. Mok CK, Boey J, Wang R, Chan TK, Cheung KL, Lee PK, Chow J, Ng RP, Tse TF. Warfarin versus dipyridamole-aspirin and pentoxifylline-aspirin for the prevention of prosthetic heart valve thromboembolism: a prospective randomized clinical trial. Circulation 1985;72:1059-1063.

181. Heneghan C, Ward A, Perera R, Bankhead C, Fuller A, Stevens R, Bradford K, Tyndel S, Alonso-Coello P, Ansell J, Beyth R, Bernardo A, Christensen TD, Cromheecke ME, Edson RG, Fitzmaurice D, Gadisseur AP, Garcia-Alamino JM, Gardiner C, Hasenkam JM, Jacobson A, Kaatz S, Kamali F, Khan TI, Knight E, Kortke H, Levi M, Matchar D, Menendez-Jandula B, Rakovac I, Schaefer C, Siebenhofer A, Souto JC, Sunderji R, Gin K, Shalansky K, Voller H, Wagner O, Zittermann A. Self-monitoring of oral anticoagulation: systematic review and meta-analysis of individual patient data. Lancet 2012;379:322-334.

182. Fiedler KA, Maeng M, Mehilli J, Schulz-Schupke S, Byrne RA, Sibbing D, Hoppmann P, Schneider S, Fusaro M, Ott I, Kristensen SD, Ibrahim T, Massberg S, Schunkert H, Laugwitz KL, Kastrati A, Sarafoff N. Duration of triple therapy in patients requiring oral anticoagulation after drug-eluting stent implantation: the ISAR-TRIPLE Trial. J Am Coll Cardiol 2015;65:1619-1629.

183. Gibson CM, Mehran R, Bode C, Halperin J, Verheugt FW, Wildgoose P, Birmingham M, lanus J, Burton P, van Eickels M, Korjian S, Daaboul Y, Lip GY, Cohen M, Husted S, Peterson ED, Fox KA. Prevention of bleeding in patients with atrial fibrillation undergoing PCl. N Engl J Med 2016;375:2423-2434.

184. Dewilde WJ, Oirbans T, Verheugt FW, Kelder JC, De Smet BJ, Herrman JP, Adriaenssens T, Vrolix M, Heestermans AA, Vis MM, Tijsen JG, van't Hof AW, ten Berg, JM, WOEST study investigators. Use of clopidogrel with or without aspirin in patients taking oral anticoagulant therapy and undergoing percutaneous coronary intervention: an open-label, randomised, controlled trial. Lancet 2013;381:1107-1115.

185. Lamberts M, Gislason GH, Lip GY, Lassen JF, Olesen JB, Mikkelsen AP, Sorensen R, Kober L, Torp-Pedersen C, Hansen ML. Antiplatelet therapy for stable coronary artery disease in atrial fibrillation patients taking an oral anticoagulant: a nationwide cohort study. Circulation 2014;129:1577-1585.

186. Torella M, Torella D, Chiodini P, Franciulli M, Romano G, De Santo L, De Feo M, Amarelli C, Sasso FC, Salvatore T, Ellison GM, Indolfi C, Cotrufo M, Nappi G. LOWERing the INtensity of oral anticoaGulant Therapy in patients with bileaflet mechanical aortic valve replacement: results from the "LOWERINGIT" Trial. Am Heart J 2010;160:171-178.

187. Puskas J, Gerdisch M, Nichols D, Quinn R, Anderson C, Rhenman B, Fermin L, McGrath M, Kong B, Hughes C, Sethi G, Wait M, Martin T, Graeve A, PROACT Investigators. Reduced anticoagulation after mechanical aortic valve replacement: interim results from the prospective randomized on-X valve anticoagulation clinical trial randomized Food and Drug Administration investigational device exemption trial. J Thorac Cardiovasc Surg 2014;147:1202-1210; discussion 1210-1201.

188. Koertke H, Zittermann A, Wagner O, Secer S, Christ of H, Sciangula A, Saggau W, Sack FU, Ennker J, Cremer J, Musumeci F, Gummert JF. Telemedicineguided, very low-dose international normalized ratio self-control in patients with mechanical heart valve implants. Eur Heart J 2015;36:1297-1305. 
189. Bussey HI, Bussey M, Bussey-Smith KL, Frei CR. Evaluation of warfarin management with international normalized ratio self-testing and online remote monitoring and management plus low-dose vitamin $\mathrm{K}$ with genomic considerations: a pilot study. Pharmacotherapy 2013;33:1136-1146.

190. Pernod G, Godier A, Gozalo C, Tremey B, Sie P, French National Authority for Health. French clinical practice guidelines on the management of patients on vitamin $\mathrm{K}$ antagonists in at-risk situations (overdose, risk of bleeding, and active bleeding). Thromb Res 2010;126:e167-174.

191. Halvorsen S, Storey RF, Rocca B, Sibbing D, Ten Berg J, Grove EL, Weiss TW, Collet JP, Andreotti F, Gulba DC, Lip GY, Husted S, Vilahur G, Morais J, Verheugt FW, Lanas A, Al-Shahi Salman R, Steg PG, Huber K, ESC Working Group on Thrombosis. Management of antithrombotic therapy after bleeding in patients with coronary artery disease and/or atrial fibrillation: expert consensus paper of the European Society of Cardiology Working Group on Thrombosis. Eur Heart J 2017;38:1455-1462.

192. Nishimura RA, Otto CM, Bonow RO, Carabello BA, Erwin JP 3rd, Guyton RA, O'Gara PT, Ruiz CE, Skubas NJ, Sorajja P, Sundt TM 3rd, Thomas JD, ACC/ AHA Task Force Members. 2014 AHA/ACC guideline for the management of patients with valvular heart disease: a report of the American College of Cardiology/American Heart Association Task Force on Practice Guidelines. Circulation 2014;129:e521-643.

193. Whitlock RP, Sun JC, Fremes SE, Rubens FD, Teoh KH, American College of Chest Physicians. Antithrombotic and thrombolytic therapy for valvular disease: Antithrombotic Therapy and Prevention of Thrombosis, 9th ed: American College of Chest Physicians Evidence-Based Clinical Practice Guidelines. Chest 2012;141:e576S-600S.

194. Massel DR, Little SH. Antiplatelet and anticoagulation for patients with prosthetic heart valves. Cochrane Database Syst Rev 2013;4:CD003464.

195. Valgimigli M, Bueno H, Byrne RA, Collet JP, Costa F, Jeppsson A, Juni P, Kastrati A, Kolh P, Mauri L, Montalescot G, Neumann FJ, Petricevic M, Roffi M, Steg PG, Windecker S, Zamorano JL, 2017 ESC focused update on dual antiplatelet therapy in coronary artery disease developed in collaboration with EACTS. The Task Force for dual antiplatelet therapy in coronary artery disease of the European Society of Cardiology (ESC) and of the European Association for Cardio-Thoracic Surgery (EACTS). Eur Heart J 2017; doi:10.1093/eurheartj/ehx419.

196. Kristensen SD, Knuuti J, Saraste A, Anker S, Botker HE, Hert SD, Ford I, Gonzalez-Juanatey JR, Gorenek B, Heyndrickx GR, Hoeft A, Huber K, lung B, Kjeldsen KP, Longrois D, Luscher TF, Pierard L, Pocock S, Price S, Roffi M, Sirnes PA, Sousa-Uva M, Voudris V, Funck-Brentano C. 2014 ESC/ESA Guidelines on non-cardiac surgery: cardiovascular assessment and management: the Joint Task Force on non-cardiac surgery: cardiovascular assessment and management of the European Society of Cardiology (ESC) and the European Society of Anaesthesiology (ESA). Eur Heart J 2014;35:2383-2431.

197. Francophone Society of Oral Medicine and Oral Surgery, with the collaboration of the French Society of Cardiology. Guidelines for management of patients under antivitamin K treatment in oral surgery (2006). http://www.mbcb-journal. org/recommendations/254-guidelines-for-management-of-patients-under-antivi tamin-k-treatment-in-oral-surgery-2006

198. Karthikeyan G, Senguttuvan NB, Joseph J, Devasenapathy N, Bahl VK, Airan B. Urgent surgery compared with fibrinolytic therapy for the treatment of leftsided prosthetic heart valve thrombosis: a systematic review and meta-analysis of observational studies. Eur Heart / 2013;34:1557-1566.

199. Laplace G, Lafitte S, Labeque JN, Perron JM, Baudet E, Deville C, Roques X, Roudaut R. Clinical significance of early thrombosis after prosthetic mitral valve replacement: a postoperative monocentric study of 680 patients. J Am Coll Cardiol 2004;43:1283-1290.

200. Brown ML, Park SJ, Sundt TM, Schaff HV. Early thrombosis risk in patients with biologic valves in the aortic position. J Thorac Cardiovasc Surg 2012;144:108-111.

201. Mylotte D, Andalib A, Theriault-Lauzier P, Dorfmeister M, Girgis M, Alharbi W, Chetrit M, Galatas C, Mamane S, Sebag I, Buithieu J, Bilodeau L, de Varennes B, Lachapelle K, Lange R, Martucci G, Virmani R, Piazza N. Transcatheter heart valve failure: a systematic review. Eur Heart J 2015;36:1306-1327.

202. Makkar RR, Fontana G, Jilaihawi H, Chakravarty T, Kofoed KF, de Backer O, Asch FM, Ruiz CE, Olsen NT, Trento A, Friedman J, Berman D, Cheng W, Kashif M, Jelnin V, Kliger CA, Guo H, Pichard AD, Weissman NJ, Kapadia S, Manasse E, Bhatt DL, Leon MB, Sondergaard L. Possible subclinical leaflet thrombosis in bioprosthetic aortic valves. N Engl J Med 2015;373:2015-2024.

203. Pache G, Schoechlin S, Blanke P, Dorfs S, Jander N, Arepalli CD, Gick M, Buettner HJ, Leipsic J, Langer M, Neumann FJ, Ruile P. Early hypo-attenuated leaflet thickening in balloon-expandable transcatheter aortic heart valves. Eur Heart J 2016;37:2263-2271.

204. Sorajja P, Bae R, Lesser JA, Pedersen WA. Percutaneous repair of paravalvular prosthetic regurgitation: patient selection, techniques and outcomes. Heart 2015;101:665-673.

205. Dvir D, Webb JG, Bleiziffer S, Pasic M, Waksman R, Kodali S, Barbanti M, Latib A, Schaefer U, Rodes-Cabau J, Treede H, Piazza N, Hildick-Smith D, Himbert D, Walther T, Hengstenberg C, Nissen H, Bekeredjian R, Presbitero P, Ferrari E, Segev A, de Weger A, Windecker S, Moat NE, Napodano M, Wilbring M, Cerillo AG, Brecker S, Tchetche D, Lefevre T, De Marco F, Fiorina C, Petronio AS, Teles RC, Testa L, Laborde JC, Leon MB, Kornowski R, Valve-in-Valve International Data Registry Investigators. Transcatheter aortic valve implantation in failed bioprosthetic surgical valves. JAMA 2014;312:162-170.

206. Ye J, Cheung A, Yamashita M, Wood D, Peng D, Gao M, Thompson CR, Munt B, Moss RR, Blanke P, Leipsic J, Dvir D, Webb JG. Transcatheter aortic and mitral valve-in-valve implantation for failed surgical bioprosthetic valves: an 8 year single-center experience. JACC Cardiovasc Interv 2015;8:1735-1744.

207. Calleja AM, Dommaraju S, Gaddam R, Cha S, Khandheria BK, Chaliki HP. Cardiac risk in patients aged $>75$ years with asymptomatic, severe aortic stenosis undergoing noncardiac surgery. Am J Cardiol 2010;105:1159-1163.

208. Tashiro T, Pislaru SV, Blustin JM, Nkomo VT, Abel MD, Scott CG, Pellikka PA Perioperative risk of major non-cardiac surgery in patients with severe aortic stenosis: a reappraisal in contemporary practice. Eur Heart J 2014;35:2372-2381.

209. European Society of Gynecology (ESG), Association for European Paediatric Cardiology (AEPC), German Society for Gender Medicine (DGesGM), RegitzZagrosek V, Blomstrom Lundqvist C, Borghi C, Cifkova R, Ferreira R, Foidart JM, Gibbs JS, Gohlke-Baerwolf C, Gorenek B, lung B, Kirby M, Maas AH, Morais J, Nihoyannopoulos P, Pieper PG, Presbitero P, Roos-Hesselink JW, Schaufelberger M, Seeland U, Torracca L, ESC Committee for Practice Guidelines. ESC Guidelines on the management of cardiovascular diseases during pregnancy: the Task Force on the Management of Cardiovascular Diseases during Pregnancy of the European Society of Cardiology (ESC). Eur Heart J 2011;32:3147-3197.

210. Elassy SM, Elmidany AA, Elbawab HY. Urgent cardiac surgery during pregnancy: a continuous challenge. Ann Thorac Surg 2014;97:1624-1629.

211. van Hagen IM, Roos-Hesselink JW, Ruys TP, Merz WM, Goland S, Gabriel H, Lelonek M, Trojnarska O, Al Mahmeed WA, Balint HO, Ashour Z, Baumgartner H, Boersma E, Johnson MR, Hall R, ROPAC Investigators and the EURObservational Research Programme (EORP) Team. Pregnancy in women with a mechanical heart valve: data of the European Society of Cardiology Registry of Pregnancy and Cardiac Disease (ROPAC). Circulation 2015;132:132-142. 